Florida International University

FIU Digital Commons

FIU Electronic Theses and Dissertations

University Graduate School

$11-12-2020$

\title{
Children's Creative Processes in Music Composition Through the Orff-Schulwerk Approach
}

Ashley Viera Espinal

Florida International University, avier026@fiu.edu

Follow this and additional works at: https://digitalcommons.fiu.edu/etd

Part of the Cognitive Psychology Commons, Educational Methods Commons, Educational Psychology Commons, and the Music Education Commons

\section{Recommended Citation}

Espinal, Ashley Viera, "Children's Creative Processes in Music Composition Through the Orff-Schulwerk Approach" (2020). FIU Electronic Theses and Dissertations. 4542.

https://digitalcommons.fiu.edu/etd/4542

This work is brought to you for free and open access by the University Graduate School at FIU Digital Commons. It has been accepted for inclusion in FIU Electronic Theses and Dissertations by an authorized administrator of FIU Digital Commons. For more information, please contact dcc@fiu.edu. 


\title{
FLORIDA INTERNATIONAL UNIVERSITY
}

Miami, Florida

\section{CHILDREN'S CREATIVE PROCESSES IN MUSIC COMPOSITION THROUGH THE ORFF-SCHULWERK APPROACH}

\author{
A thesis submitted in partial fulfillment of \\ the requirements for the degree of \\ MASTER OF SCIENCE \\ in \\ MUSIC EDUCATION
}

by

Ashley Viera Espinal 
To: Dean Brian Schriner

College of Communication, Architecture + The Arts

This thesis, written by Ashley Viera Espinal, and entitled Children's Creative Processes in Music Composition Through the Orff-Schulwerk Approach, having been approved in respect to style and intellectual content, is referred to you for judgment.

We have read this thesis and recommend that it be approved.

\author{
Sandra Adorno
}

Melody Whiddon Willoughby

Candice Davenport Mattio, Major Professor

Date of Defense: November 12, 2020.

The thesis of Ashley Viera Espinal is approved.

Dean Brian Schriner
College of Communication, Architecture + The Arts

Andrés G. Gil

Vice President for Research and Economic Development and Dean of the University Graduate School

Florida International University, 2020 


\section{DEDICATION}

This thesis is dedicated to my loving parents, grandmother in heaven, and amazing

husband who have been my support system throughout this entire process and a positive

influence in my life, now and forever. 


\section{ACKNOWLEDGMENTS}

This thesis could not have been possible without the help of so many wonderful people. I would like to extend my gratitude to God, my professors, family, and furbabies that have provided so much love, support, and encouragement throughout this journey.

First and foremost, thank you God for providing me with the strength and courage to endure the obstacles I encountered along the way towards completing my thesis.

A big and warm thank you to my loving parents Miguel and Amelia Viera, whose love and support I have had since day one. They always believed in my potential to succeed and be a great music educator. Thank you for teaching me the true value of hard work and dedication.

To my bright and cheerful Mima. Words cannot express how much she has been missed throughout the years, but knowing that she was always there watching over me and cheering me on from heaven brought hope and warmth into my heart in times of need.

To my loving and ever-so-needy furbabies Emerson, Grayson, Abigail, and Peaches. Thank you guys for reminding me of your unconditional love every time you guys would bump your heads against my leg or forearm while I typed away on the computer. I am truly grateful to have you guys in my life.

To my committee members Drs. Adorno, Berglin, Davenport, and Willoughby. Thank you for your expertise, endless support, and insightful efforts towards this lifechanging experience. A special thank you to Drs. Adorno and Davenport for helping me grow as a student and researcher throughout these last few years of graduate school, and for all the shared jokes and laughter we had along the way. 
No words are sufficient to express my immense gratitude to Dr. Candice Davenport. Her genuine support, encouragement, and boundless efforts in shaping me as a researcher has truly been an honor. I will forever be grateful for her mentorship, thoughtfulness, and friendship.

Lastly, the biggest and warmest thank you to my wonderful husband, Diego. He believed in me even when I did not believe in myself throughout this entire process.

Thank you for embracing the long and tumultuous road that was my thesis alongside me, and for always providing me with your boundless love and support in all that I do. I am forever grateful for all that you are and all that you do. I love you honey, always and forever! 


\title{
ABSTRACT OF THE THESIS \\ CHILDREN'S CREATIVE PROCESSES IN MUSIC COMPOSITION THROUGH \\ THE ORFF-SCHULWERK APPROACH
}

\author{
by \\ Ashley Viera Espinal \\ Florida International University, 2020 \\ Miami, Florida \\ Professor Candice Davenport Mattio, Major Professor
}

The purpose of this descriptive qualitative study was to explore the creative processes of elementary school students in music composition through the OrffSchulwerk approach. Twenty-two 5th grade students and a music teacher with all three levels of the Orff-Schulwerk certification participated in the study. There were three main focal points that were investigated within a span of five-weeks: 1) children's creativity in an Orff-based composition task, 2) children's perceptions of their creative processes employed during the composition task, and 3) the teacher's perceptions of teaching/learning music composition through the Orff-Schulwerk approach. Data were generated through observations, field notes, interviews with the focus group and teacher, audio material, and collected artifacts regarding the compositional task and lesson plan. Results of the study suggested that children's creative processes within this approach's implementation progressed in an interchangeably cyclical manner, and enabling skills/conditions also play a factor in influencing their creative processes. 


\section{TABLE OF CONTENTS}

CHAPTER

PAGE

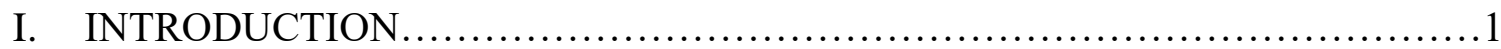

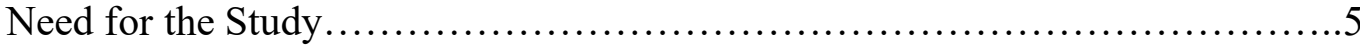

Purpose and Research Questions.........................................6

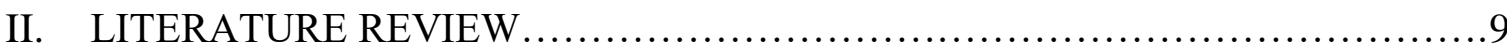

Creative Thinking in Music............................................. 9

Creativity and Composition in the Elementary Music Classroom..................11

Creativity and Composition in the Orff-Schulwerk Approach....................14

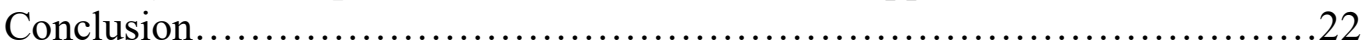

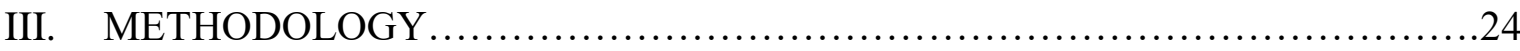

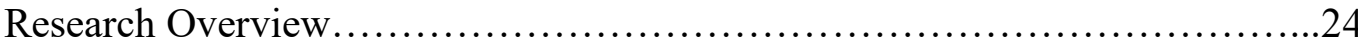

Central Purpose and Research Questions...................................26

Descriptive Qualitative Research........................................26

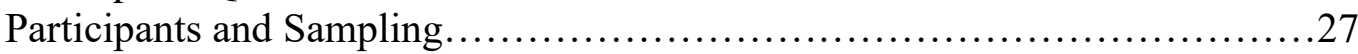

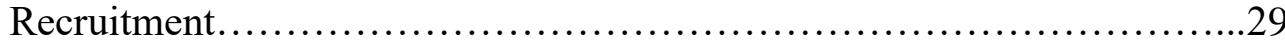

Consent and Assent......................................................... 30

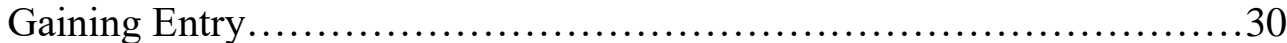

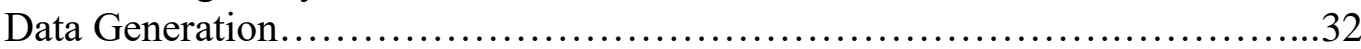

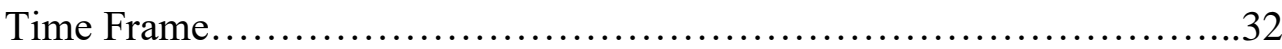

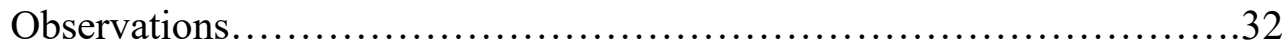

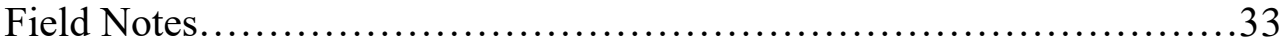

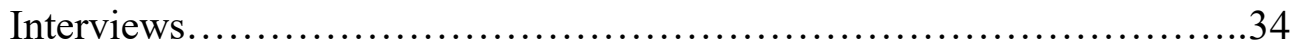

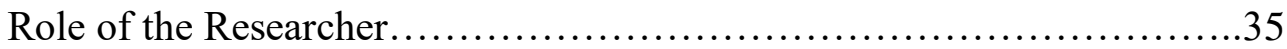

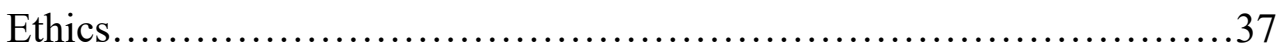

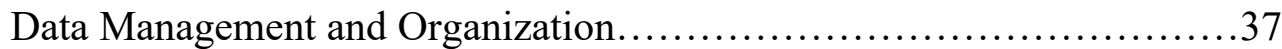

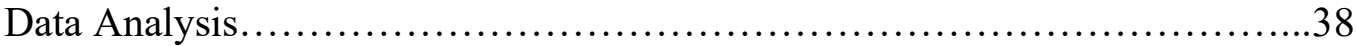

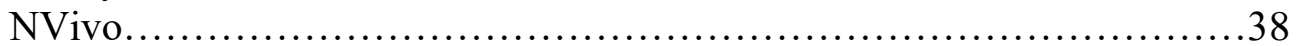

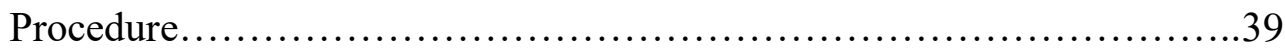

Theoretical Framework............................................ 40

Validation of Analysis................................................... 41

IV. ANALYSIS AND FINDINGS..................................................42

Creative Compositional Process................................................44

Annie's Group ......................................................... 44

Bassline......................................................... 44

Melody ..................................................... 48

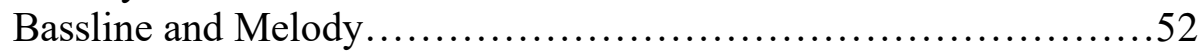

Color Part.............................................................

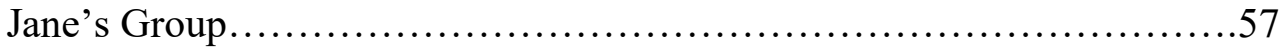

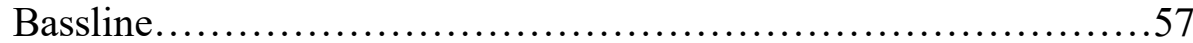


Melody ......................................................... 60

Bassline, Melody, and Color Part.................................. 71

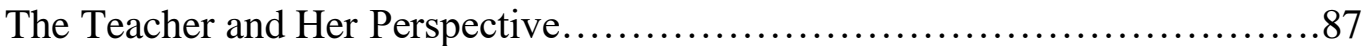

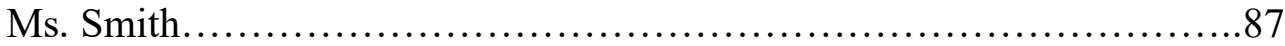

Warm-ups.................................................... 87

Teaching Process.................................................. 89

Orff-Schulwerk.................................................. 92

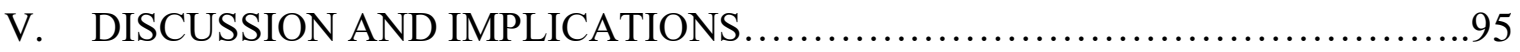

Restatement of Purpose and Research Questions.............................95

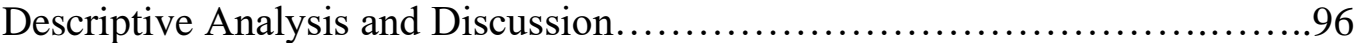

Research Question One..........................................96

Bassline.............................................................. 96

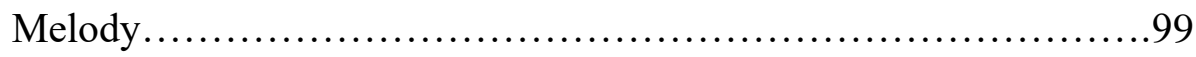

Color Part......................................................

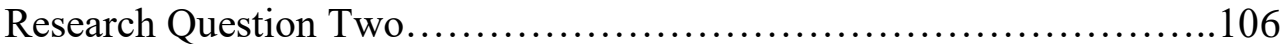

Enabling Conditions..........................................106

Research Question Three...........................................109

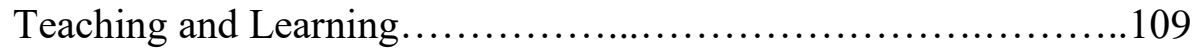

Conclusion of Discussion...........................................112

Suggestions for Practitioners.............................................114

Suggestions for Future Research......................................116

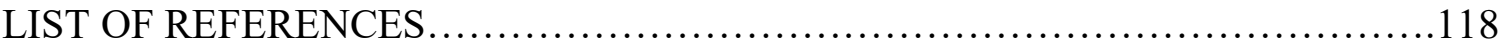

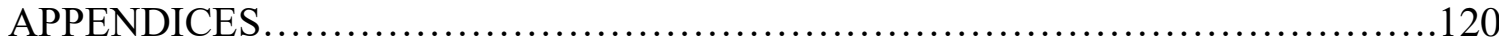




\section{LIST OF FIGURES}

FIGURE

PAGE

Figure 1.1. Model of Creative Thinking in Music..................................5

Figure 4.1. Melody Rhythm Options.......................................49

Figure 4.2. Melody of Annie's Group.........................................51

Figure 4.3. Bassline of Annie's Group............................................53

Figure 4.4. Bassline of Jane's Group........................................58

Figure 4.5. Melody of Jane's Group........................................ 75 


\section{CHAPTER I:}

\section{INTRODUCTION}

Influenced by the eurhythmic principles of Émile Jaques-Dalcroze, German composer Carl Orff believed children could develop musical attributes through the harmonious experience and exploration of music before its formal introduction (Mark \& Gary, 2007; Orff \& Walter, 1963; Thresher, 1964; Wang \& Springer, 2013). Children surrounded by joyful and explorative atmospheres are presumed to learn music, especially if permitted to do so naturally and spontaneously (Wang \& Springer, 2013). This presumption was the basis for Orff's ideology to the fundamental conceptualization of elemental music associated with children's musical learning, claiming that “... almost every child can comprehend and enjoy music." When children experience and explore music through movement, dance, and speech, it serves as an introduction which facilitates their musical understanding and learning (Orff \& Walter, 1963, p. 72).

As the connection between music, movement, and speech was considered the foundation towards elemental music learning, Orff reformed his former approach to tailoring its pedagogical model for children's fundamental learning, henceforth known as the Schulwerk. Orff, in collaboration with Gunild Keetman, published several volumes of his Schulwerk (known as Music for Children) containing a compilation of musical examples and references, serving as pedagogical guides for teachers to facilitate musicmaking activities for children's music learning (Frazee \& Kreuter, 1987; Orff \& Walter, 1963; Thresher, 1964; Wang \& Springer, 2013). Schulwerk had appealed to both national and international institutions (Mark \& Gary, 2007; Orff \& Walter, 1963), particularly in North America due to its elemental music-making accessible to all age-ranges or 
experiential levels within music, which led to various workshops, demonstrations, and courses of the approach being held across the United States (Wang \& Springer, 2013).

By the late 1960s, Schulwerk had already been adopted and subsequently integrated into American schools throughout the United States due to its global prominence (Mark \& Gary, 2007). Schulwerk differed from its predecessor because it pertained to children's prior musical exposure as a proxy to their creative music-making, gradually expanding children's musicality and musical creativity throughout their progressive musical training (Orff \& Walter, 1963; Thresher, 1964). In its latest iteration, the Orff-Schulwerk approach is geared towards children's musical ability by focusing on the fundamental elements of their music-making and creativity through listening, singing, moving, and playing instruments (Beegle \& Bond, 2016; Wang \& Springer, 2013; Womack, 2008). With improvisation and creativity being the main foci throughout the approach, children's fundamental learning of music concepts are facilitated through the elemental music-making process while simultaneously enhancing their creative musicianship (Shehan, 1986).

The Orff-Schulwerk approach has gained worldwide recognition since it arrived in the United States. Its fundamental approach has demonstrated to be a valuable contribution towards the development of children's learning in music education. More specifically, the process of music-making within the Orff-Schulwerk approach has proven to be useful towards children's musical experiences and fundamental music learning, stimulating their creative ability and potential profoundly (Choksy, Abramson, Gillespie, Woods, \& York, 2001; Churchley, 1980; Hickey, 2003, 2012; Orff \& Walter, 1963; Shehan, 1986; Thresher, 1964; Wang \& Springer, 2013). 
Thresher (1964) states, "Carl Orff”s philosophy is that music education, first of all, should develop the child's ability to create, or in the musical idiom, to improvise" (p. 44). Conforming to Webster's model of creative thinking (1990), the Orff-Schulwerk approach encourages children to immerse themselves in "playful musical exploration" and "creative expression" (Beegle \& Bond, 2016) while they navigate through their creative thought processes. Children develop their creative thought processes as they move through the stages of divergent-to-convergent thinking, specifically as they work through their musical preparation, respite, compilation, and demonstration of their musical intention through music-making (Wang \& Springer, 2013). Children can freely express their musical thinking while demonstrating conceptual music understanding through composition (Wiggins, 2003). The subsequent act of composing and improvising is essential in stimulating children to develop their musical identity. Children's musical experiences and musicality can be augmented through composing and improvising while also inspiring their creative novelties (Hickey, 2012). This process allows children's compositional outcomes to be reached as they exhibit their musical and creative inclination. These skills are based on several determinants, including music, aesthetics, cognition, craftsmanship, and internal and external conditions (Webster, 1990). They are reflected through the core stages of divergent and convergent thinking.

As children discover their creative processes in music composition through the Orff approach, music educators can enhance their composition approaches that nurture and facilitate children's independent thinking. In this way, educators can also encourage creative authenticity through music-making (Hickey, 2003, 2012). The more opportunities children have to compose within specified guidelines (e.g., using particular 
scales or rhythmic patterns), the more likely they are to develop gratifying end-results to their musical compositions while inadvertently enhancing their creativity. Furthermore, children can also demonstrate their divergent and convergent thought processes when they are given substantial opportunities to compose musically through hands-on exploration (Wang \& Springer, 2013), which is an elemental characteristic of the OrffSchulwerk approach.

Webster (1990) defines musical creativity as "the mental process that alternates between divergent (imaginative) and convergent (factual) thinking, moving in stages over time... enabled by internal musical skills and outside conditions and results in a final musical product which is new for the creator" (p. 28). Further, Wiggins (2003) explains that creativity within music composition is "generating musical ideas (motivic material and musical gestures) and setting them into a context" (p. 141), and that contextualizing musical ideas to further one's composition interacts between and among the different phases of their compositional stages such as organizing, evaluating, revising, refining, and rehearsing. Musical creativity can also be thought of as the ability to articulate significant musical ideas through music-making by demonstrating one's musical inclination based on the determinants of music, aesthetics, cognition, craftsmanship, and internal and external conditions, which cumulatively result in a creative product (Webster, 1990; Wiggins, 2003).

Explicitly, for the sake of this study, the creative processes in children's musical compositions will be examined through the framework of Webster's (1990) model of creative thinking in music (See Figure 1.1). Since this study seeks to investigate the processes of musical creativity, the core stages of moving through divergent to 
convergent thinking (including the stages of preparation, incubation, illumination, verification; Webster, 1990), will be used as the basis of analysis in this study.

\section{Figure 1.1.}

\section{Model of Creative Thinking in Music}

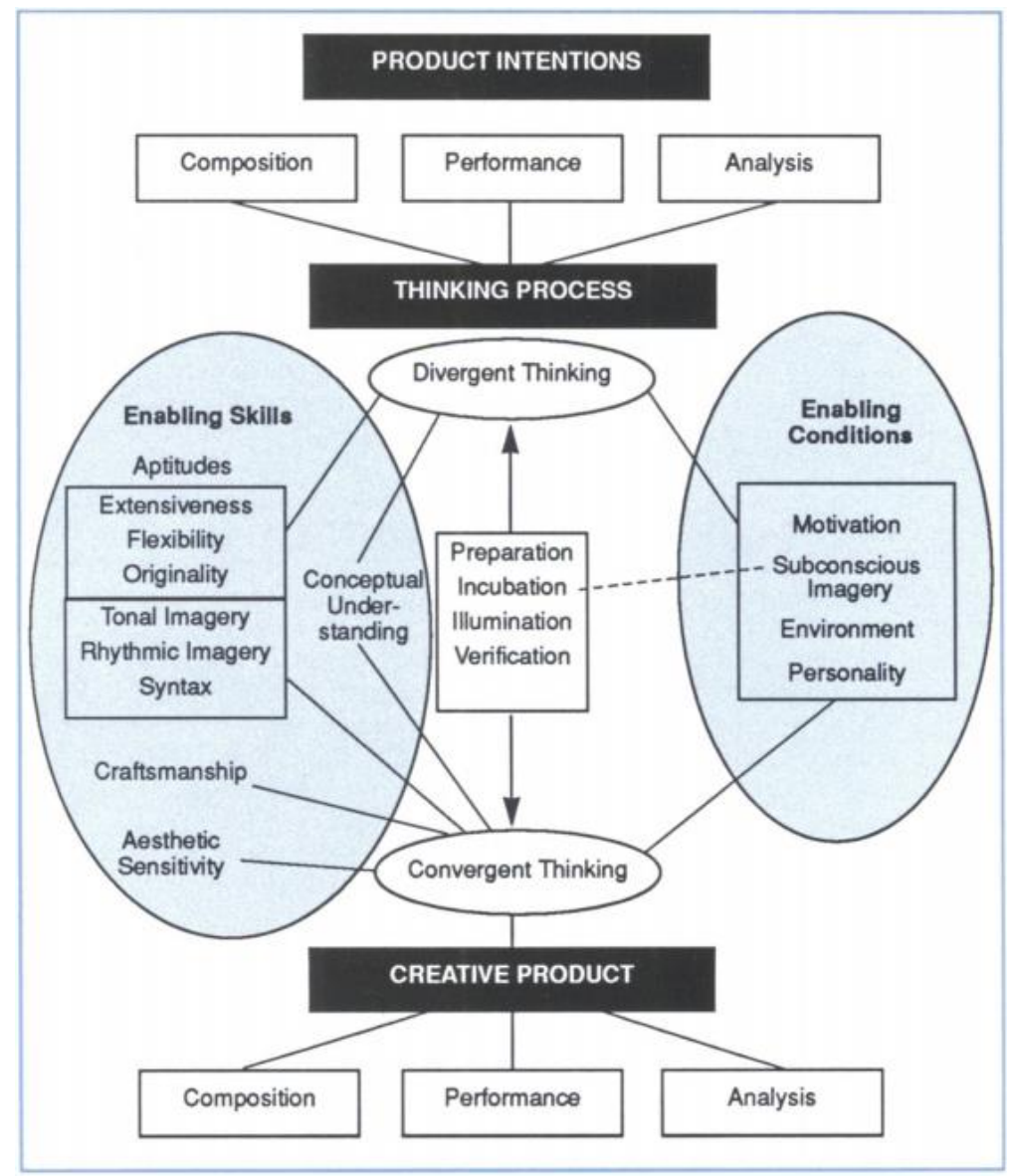

Note: Webster's (1990) Model of Creative Thinking in Music (p. 23).

\section{Need for the Study}

The process of music-making within the Orff-Schulwerk approach has proven to be useful towards children's musical understanding and learning (Choksy et al., 2001; Churchley, 1980; Hickey, 2003, 2012; Orff \& Walter, 1963; Shehan, 1986; Thresher, 1964; Wang \& Springer, 2013). There is evidence to support the recognition of the Orff- 
Schulwerk approach within music education in regards to its music-making process. However, current academic research suggests that there is an insufficient amount of data on the thought process of musical creativity in music composition through the approach's implementation (Hickey, 2003, 2012; Kratus, 1989; Orff \& Walter, 1963; Thresher, 1964; Wang \& Springer, 2013; Webster, 1990; Wiggins, 2003; Womack, 2008). Further knowledge of children's musical creativity through the compositional process of the approach could contribute to a better understanding of children's creative thought processes and enhance teaching strategies with regards to musical creativity.

\section{Purpose and Research Questions}

The purpose of this study is to explore the creative processes of elementary school students in music composition in an Orff-based music class. Specifically, this study will examine their creative processes through the framework of divergent to convergent thinking within Webster's (1990) model of creative thinking in music. The following central questions were used to guide this study:

1. What is the nature of children's creative processes during an Orff-based music composition task?

2. What are the children's perceptions of their own creative processes employed during an Orff-based composition task?

3. What are the teacher's perceptions of teaching and learning music composition through the Orff-Schulwerk approach?

By examining children's creative processes, researchers strive to understand children's creative thinking as they depict musical and creative skills through various developmental stages. Describing children's creative compositional processes within an 
Orff-based music classroom could enhance the instructional curricula and implementation within music education more effectively. Additionally, research supports that children who are given numerous opportunities and encouraged to compose musically can enhance their creative processes, thus transitioning to their compositional products. These opportunities also demonstrate that children's creative thought processes alternate between divergent and convergent thinking through their musical composition (Wang \& Springer, 2013). By allowing children to depict musical and creative ideas through the elemental music-making process within the Orff approach, both practitioners and researchers can educate themselves more on children's creative processes during creative musical tasks.

Examining the process of music-making in the Orff-Schulwerk could be beneficial to teachers and researchers as it contributes to the development of children's musical experiences, musical abilities, and fundamental music learning, which could lead to children's musical and creative growth. Furthermore, by understanding the approach's music-making process, teachers can evolve their teaching strategies that could promote children's creative abilities and potential by enlightening and stimulating them as they continue to grow music-wise. Moreover, by examining the music-making process in the Orff-Schulwerk approach in tandem to the creative processes through a qualitative perspective can provide a subjective outcome that is equally as important as an objective outcome like children's creative products, especially with children's creativity moving on a creative range. This lends itself to an open-mindedness towards proceeding with assessing children's creativity through a subjective way that many practitioners and 
researchers alike do neglect by the given nature of assessing the creative product versus the creative process. 


\section{CHAPTER II:}

\section{LITERATURE REVIEW}

Children's musicality and musical experiences have been substantiated through an abundance of literature review on the Orff-Schulwerk approach and its elemental musicmaking process (Choksy et al., 2001; Churchley, 1980; Hickey, 2003, 2012; Orff \& Walter, 1963; Shehan, 1986; Thresher, 1964; Wang \& Springer 2013). However, current academic research suggests insufficient data on the thought process of musical creativity in music composition through the approach's implementation (Hickey, 2003, 2012; Kratus, 1989; Orff \& Walter, 1963; Thresher, 1964; Wang \& Springer, 2013; Webster, 1990; Wiggins, 2003; Womack, 2008). While there is a substantial amount of evidence supporting the music-making process of its implementation in regards to the development of children's musical aptitude, further knowledge on children's musical creativity through the compositional process of the approach could suffice a better understanding of children's creative processes in the Orff-Schulwerk approach. Therefore, this chapter will provide a synthesis of research literature relating to creative thinking in music, creativity and composition in the elementary music classroom, and creativity in the Orff-Schulwerk approach. An overview of the relevant research literature might help the reader understand the major theories and models that led to the conceptual thought behind the design and rationale of the present study.

\section{Creative Thinking in Music}

Webster (1990) defines musical creativity as "the mental process that alternates between divergent (imaginative) and convergent (factual) thinking, moving in stages over time... enabled by internal musical skills and outside conditions and results in a final 
musical product which is new for the creator" (p. 28). Additionally, Arieti (1976) in Creativity: The Magic Synthesis defines creativity as:

One of the major means by which the human being liberates himself from the fetters not only of his conditioned responses, but also of his usual choices. However, creativity is not simply originality and unlimited freedom. There is much more to it than that... [it] also imposes restrictions. While it uses methods other than those of ordinary thinking, it must not be in disagreement with ordinary thinking - or rather, it must be something that, sooner or later, ordinary thinking will understand, accept, and appreciate. Otherwise the result would be bizarre, not creative. (p. 4)

Webster's (1990) Model of Creative Thinking in Music is a model that illustrates the interrelationship between components that function together to influence creative musical thinking. The model identifies three main components of creative musical thinking: product intentions, thinking process, and creative products. The model begins with the product intention where a specific goal (e.g., composing, performing, or musically analyzing) is set out to be achieved. Still, the goal also serves as the final creative product based on the model. The center of the model involves depicting the thinking process itself, which moves from divergent to convergent thinking. More specifically, this movement of divergent to convergent thinking goes through the stages of preparation, incubation, illumination, and verification. In addition, Webster suggests considering "enabled skills" and "enabled conditions" as factors to the alternation between divergent and convergent thinking. These skills are distinguished by musical and creative proficiency, including determinants of music, aesthetics, cognition, and 
craftsmanship. The conditions are based on motivation, subconscious imagery, personality, and environment but are considered beneficial towards one's musical and creative proficiency. The final creative product results from the depiction of the thinking process within the enabled skills and conditions. Webster's model is a multicomponential framework that is efficient to evaluate musical creativity (Davenport, 2016). Teachers and researchers alike can use the framework to further understand how creativity works in regards to musical intentions.

Creativity can be described as balancing one's mental thought processes without fully disregarding nor blindly accepting the outcome of those thoughts. Much like creativity, musical creativity is the mental process that travels between divergent-toconvergent thinking, specifically moving through the stages of preparation, incubation, illumination, and verification. Additionally, indications of enabling skills and enabling conditions can also be contributing factors towards one's creative thought processes, which can negatively or positively impact their musical intentions.

\section{Creativity and Composition in the Elementary Music Classroom}

Wiggins (2003) states that creativity within music composition can be viewed as "generating musical ideas (motivic material and musical gestures) and setting them into a context" (p. 141). In her chapter titled "A Frame for Understanding Children's Compositional Processes," Wiggins (2003) elaborates on an ethnographic study conducted in 1990 to investigate students' compositional processes through their experiences in performing, listening, and creating music. The researcher [Wiggins] had continuously analyzed the transcribed data from audio/video tapes while gathering data throughout a 5-month data-collection period. Wiggins further elaborated that although 
collecting and analyzing data simultaneously tampered with her teaching decisions throughout the process, the overarching knowledge she gained had nourished her understanding of her own teaching approach. In addition, her continuous research on children's creativity in composition led to the synthesized model known as the frame, a compilation of characteristics describing the method to which children compose music alone, in small groups, and in whole-class settings for their original or collaborative compositional product.

Wiggins (2003) elaborated on the model called the frame by describing its process. Initially, the frame begins with children developing their musical idea(s), which is produced by allowing them to designate roles to one another, choose from a variety of instruments within the classroom (sound sourcing), choose the text if writing a lyrical song (songwriting), and the subsequent act of creating the music. Once their musical $\operatorname{idea}(s)$ is developed, children can contextualize the ideas to further their compositional stage by organizing their composition. The frame's final process is for children to perform their compositional products and receive feedback from their teachers and peers. Wiggins further elaborates that developing musical ideas differ from children's labor and collaborative labor; working alone allows children to depict their understanding of music versus collaboratively working in a group that provides a shared musical understanding as their compositional product progresses. Additionally, Wiggins states that the various aspects of the frame overlap, further representing the interactions between and among the different phases. Furthermore, the researcher [Wiggins] states that due to external influences and social context, including compositional problems, instructional 
curriculum, musical cultures, environmental surroundings, and time constraints, tampering with children's processes through composition is problematic.

Kratus (1989) examined the compositional processes of children between the ages of 7 and 11. These children participated in their general music classes for thirty-minutes twice a week, and no compositional activities were included during their class time. Children were given ten minutes to compose a song using an electric keyboard. Each child was individually tape-recorded to record the progression of their music-making during those ten minutes. Upon collecting the study's data, recordings were analyzed by coding each ten-minute session into 5-second intervals to examine their music-making based on the determinants of exploration, development, repetition, and silence (i.e., pause) while they composed their songs. Kratus (1989) found that the younger children spent most of their time exploring to compose their songs during the ten-minute sessions. The older children equally divided their music-making time among the three compositional processes (i.e., exploring, developing, and repeating) to compose their songs. Kratus (1989) also expressed that the younger children very rarely exhibited compositional processes that involved developing or repeating musical ideas, which means that their "creative act of composition... was very similar to the act of improvisation" (p. 17). Kratus (1989) also determined that the older children exhibited compositional processes similar to adult composers, but cannot manipulate these processes like adult composers due to their lack of enabled skills.

Furthermore, children were asked to perform their "final song" two times, one after the other, at the end of their ten-minute sessions, and both performances were compared to indicate similarities among the two-song performances using a three-point 
scale; three-points indicated a high level of congruence between the two performances, two-points indicated a medium level of congruence between the two performances, and one-point showed a low level of congruence between the two performances. Kratus (1989) found that the children with a three-point score invested half the time working on the repetition process to similarly perform their musical composition both times, spending less time exploring and subsequent time developing their composition. Children who scored one-point invested about three-quarters of their time exploring their musical ideas and less on any other compositional process, not providing any specific compositional intention to their "final songs" to which they could identically perform twice. Kratus (1989) concluded that as children grew older, they spent more time on the compositional development and repetition processes and less on exploration. The compositional processes present among these children support three of the stages in Webster's (1987/1990) model: exploration is indicative of preparation, development is indicative of incubation, and repetition is indicative of verification. Younger children are more likely to spend more time exploring and less time developing and repeating musical ideas due to "their interest was directed toward the process of making sounds rather than toward the creation of a single product of a composed melody" (p. 18), while also lacking in retainment of information for an extended period, difficulty to strategize musical patterns to be developed, and failure to problem-solve while composing (Kratus, 1989).

\section{Creativity and Composition in the Orff-Schulwerk Approach}

The Orff-Schulwerk approach currently focuses on the fundamental elements of music-making and creativity through singing, dancing, and playing instruments (Womack, 2008). Its primary focus is on improvisation and creativity, which facilitates 
children's fundamental learning of music concepts through its music-making process while simultaneously enhancing their creative musicianship (Shehan, 1986). By exploring the compositional process in the Orff-Schulwerk approach, researchers and practitioners alike can conclude that creativity in composing is tangentially related to the creative process in composition through the Orff-based approach. However, before having the ability to understand the compositional process within the Orff-Schulwerk approach, one must understand its approach and how it contributes to children's fundamental music learning.

In his article titled "The Schulwerk-Its Origin and Aims," Orff (1963) states that through his reestablished Schulwerk approach:

[He understood] that the unity of music and movement [was] naturally present in the child.... [and he] began to see things in the right perspective. "Elemental" was the password, applicable to music itself, to the instruments, to forms of speech and movement. [but] what does it mean?.... What, then, is elemental music? [Elemental music is music that is never alone] but music connected with movement, dance, and speech-not to be listened to, [but] meaningful only in [the] active participation. (p. 72)

Conjointly, Thresher (1964) states in her article titled "The Contributions of Carl Orff to Elementary Music Education" that:

[...] Music education, first of all, should develop the child's ability to create or, in the musical idiom, to improvise. The child must be helped to make his [or her] own music, which grows out of his [or her] own experiences in speaking and singing, moving, dancing, and playing.... Orff's belief [in] that children should be 
allowed to discover music by themselves... [could encourage their] original creative expression. (p. 44)

Tying in with Orff's (1963) and Thresher's (1964) articles, Springer (2013) discusses how improvisation and composition are the most conspicuous products of creative thinking, prudent to the Orff approach. The researcher [Springer] further states that "student's ability to generate musical material spontaneously represents the highest level of musical understanding, and as a result, improvisation is particularly emphasized in Orff-Schulwerk" (p. 174). The Orff approach stresses the experiential learning process, a multimodal and hands-on approach that engages students to experience music in an interactive way that encourages their creative thinking. This way, the pedagogical model of the Orff-Schulwerk focuses on processes of imitation, exploration, and improvisation that not only sparks children's musical experiences but also illuminates their creative thinking. Further, Springer (2013) elaborates that children's divergent and convergent thinking are enhanced through the deliberate and sufficient exploration of Orff instrumentation, which aids in "the development of creative thinking [of children] by providing numerous opportunities for sound exploration" (p. 177).

Tangentially related, Frazee and Kreuter (1987) discuss the importance of improvisation and composition on Orff instrumentation to convey children's creativity. The researchers [Frazee and Kreuter] state that classroom instruments can facilitate music-making activities through sound recognition while reinforcing rhythmic coding. Children can layer multiple rhythms to create a rhythmic or melodic composition. In addition, Frazee and Kreuter (1987) state that "Orff instruments provide an invaluable means of exploring all of the elements of music" which "offers no greater reward than the 
recognition by a child's peers of his or her unique and important [musical] creation" ( $\mathrm{p}$. 21). Furthermore, the researchers [Frazee and Kreuter] elaborate that children who improvise are "inventing new musical ideas" to which they then demonstrate "their musical independence" (p. 31). Through this musical exploration, children are encouraged to partake in the compositional process in which they "[experiment] with [musical] changes in such expressive elements [such] as dynamics, accent, color, and tempo" (p. 29), among other musical elements.

Respectively, Landis and Carder (1972) thoroughly elaborate on children's creativity through improvisation and Orff instrumentation. The researchers [Landis and Carder] state that through the Orff approach:

Creativity takes the form of improvisation.... students create rhythmic and melodic patterns, accompaniment figures, introductions, and codas [to] songs... [where] the goal of extemporaneous performance is rather to form the habit of thinking creatively.... [and by encouraging] creativity on the part of each child... [teachers provide] conditions and opportunities for [children's] improvisation [to take its course]. (pp. 86-87)

Additionally, Landis and Carder (1972) further elaborate that as children build on their improvisations, they are encouraged to use compositional devices such as borduns, ostinatos, and rondos, on specific Orff instruments which "offer[s] an interesting range of possibilities for playing [and improvising] the types of musical patterns" (p. 87) on which improvisation is solely based. Furthermore, this allows children to "play rhythmic and melodic figures and learn to manipulate them in many ways" (p. 96) to convey their musical ideas, enhancing their creativity. 
Shamrock (1986) evaluated the Orff-Schulwerk approach and discussed its influence on elementary music education. Shamrock states that the Schulwerk is a pedagogical design for teachers to guide children through their music-making process. This music-making process includes exploring, imitating, improvising, and creating music, movement, speech, and drama, starting from the fundamental element and working towards more complex components. Children engaged in this music-making process can "sing, move, play instruments, use speech in rhythmic and dramatic contexts, improvise simply in all of these areas, and combine materials into original forms" (p. 52) while improving their musical development through the approach's implementation. In addition to children's musical development, children with an Orff background can quickly adapt to the new process of music-making in secondary music education due to the approach's contribution towards providing a well-rounded musical experience to each child at the elementary level. Shamrock concludes the article by emphasizing that through the Orff-Schulwerk framework, children and teachers alike have the opportunity to explore, discover, and develop musically through singing, dancing, saying, and playing together.

Shenan (1986) evaluated multiple teaching approaches for elementary music education and described the similarities. Shenan (1986) stated that every music teaching approach equally values musical development, despite their distinction in methods and techniques. Throughout the centuries, methodologies have commonly advocated for children's experiential learning and favored music-making through children's involvement in the process. This advocacy of exploratory experiences in children's music learning became the springboard to a wide range of methodologies around the world, 
promoting children's explorative learning, namely the approaches of Orff, Dalcroze, Kodaly, Suzuki, and Gordon. Although these worldwide approaches share the common interest of children's developed musicianship, each method reaches its goals differently through their different practices and techniques. For example, Shenan describes the OrffSchulwerk approach as "an experimental form of music learning through creative play" (p. 29), which connects music with movement and speech to aid in developing children's creative musicianship. Evidence supporting the approach's contribution to children's music experience and learning can be seen in the observation of Margaret Siemens on Orff-trained fifth graders' attitudes and achievements. Siemen observed the results of fifth-grade students who were trained in the Orff method for one year concerning their musical achievement and attitudes. Siemens concluded that although traditionally trained children performed better with technique, children trained in Orff demonstrated better attitudes towards music (p. 31).

In contrast, Muriel Bebeau combined techniques from the Orff-Schulwerk and Kodaly method in speech cues to rhythm-reading instructions. A verbal cue with the same duration of a note or rest value (e.g., "wa-ter-me-lon" for a whole-note) was paired to the symbol, and movement was also employed in the study. Bebeau concluded that third graders favored the combination of Orff and Kodaly methods to speech cue rhythmreading than the traditional rhythmic counting method in two separate experiments. The emphasis of these approaches is the process of children's music-making, where children's musicianship is achieved by allowing them to experience music in various contexts and engaging them within the process of music-making itself. 
Long (2013) taught two different lesson plans using the Orff Approach within a third and sixth-grade classroom. This study aimed to examine the effectiveness of the Orff approach within a classroom setting and observe students' abilities during Orff-based activities. The "Anticipatory Set" section of the lesson plan served as an activity to introduce rhythm and beats; during this portion of the study, both groups clapped on every beat, stomped on beat one, and rhythmically vocalized fruit names to distinguish the different beat patterns. Both classes proceeded to learn the song in their distinctive lesson plan; the third graders sang "One Bottle O' Pop" while the sixth-graders learned "Black Bats." Upon learning the songs, each class was divided into groups; the third graders were divided into three groups, while sixth-graders were split into four groups. Third-grade groups sang each verse of the song in round rotations, clapping on every beat and stomping on strong beats, which in this song was beat one. Sixth-grade groups performed each line chanting and audiating with body percussion and percussion instruments. At the end of their forty-minute lesson plans, both classes successfully demonstrated strong beat, weak beat, and simple rhythms; sixth graders were able to explain the difference between strong and weak beats. In addition, both classes were able to list different reasons for the difficulty of expressing musical independence within the lesson plans. Further, the sixth-grade class was able to identify the term "audiate" and explain its definition at the end of the lesson. Long concluded her study by stating that teachers can teach children musical independence and improvisation using the Orff approach within their classrooms. Incorporating the approach into their classrooms gives students ample opportunities to explore musical creativity as they musically succeed. 
Miner (2007) investigated particular ways that foster children's musical creativity in a naturalistic manner, specifically their music-making. Miner examines the Kodaly, Orff, and Gordon approaches in their implementations' effectiveness in fostering musical creativity. Since the Kodaly and Orff approaches do not have published teacher's manuals or curricula, Miner examined their implementations through the use of distinctive writings and websites of the two approaches and corresponded with experts of their practice. The teacher manual and curricula of Jump Right In by Gordon and Woods (1992) was also investigated. Miner concluded that all three approaches have elements that benefit children in their development of musical creativity. However, his investigation determined that Jump Right In curricula was the most reflective of audiation and musical creativity development out of the three. The curriculum of Jump Right In focuses on children learning individual music patterns and complete songs in a natural sequence while promoting active music-making through improvisation and composition. The Kodaly approach builds on children's audiation skills by not sequencing musical patterns, but by providing children with ample time to learn fewer words and patterns through their elementary years. The Orff approach does not teach melodic patterns for audiation development because children already possess internal structures of music, according to its philosophy, and there is little thought on what patterns to teach and in what sequence. Miner concluded by stating three major procedures can foster musical creativity in the elementary classroom:

1. Aiding children's development in musical patterns vocabulary through exposing them to age-appropriate songs and chants and individual music patterns. 
2. Engaging children in improvisational and compositional projects through a semistructural instruction.

3. Providing children these activities and skills in a safe and accepting environment where more acceptance is provided can lead to better creative musical outcomes.

\section{Conclusion}

This section sought to present a literature synthesis that provides the reader with research-based information on the compositional process through the Orff-Schulwerk approach. The literature provided conveys an understanding of creativity in composition, specifically the creative process within music composition and the stages of divergent thinking to convergent thinking through preparation, incubation, illumination, and verification (Webster, 1990). It is evident that, based on the research literature provided in the present chapter, that researchers and practitioners alike agree that musical creativity (e.g., Arieti, 1976; Webster, 1990) is enhanced through the compositional process (e.g., Hickey, 2003, 2012; Wiggins, 2003). The compositional processes through Orff-based (e.g., Frazee \& Kreuter, 1987; Landis \& Carder, 1972; Springer, 2013) facilitate children's fundamental music learning and creativity (Orff, 1963; Thresher, 1964).

Hopefully, this synthesis of research literature was informative so that the reader may better understand the major theories and models that led to the design and rationale of the present study. The literature presented in the current chapter supports examining children's creativity in composition, more specifically the compositional processes through Orff-based approaches. Current studies tend to recognize the inherent value of the Orff-Schulwerk approach in regards to its music-making process. However, there is a 
lack of research investigating the thought process of children's musical creativity in Orffbased music composition tasks, particularly as defined stages of preparation, incubation, illumination, and verification. Such investigations can contribute to an in-depth

understanding of children's creative processes as well as enhancing teaching approaches with regards to musical creativity. 


\section{CHAPTER III:}

\section{METHODOLOGY}

This chapter will describe the research methodology, design, and procedures.

First, I will present a brief overview of the study, along with a restatement of the purpose and research questions. Next, I will provide the methodological framework used for this study and provide details regarding participants' recruitment and sampling, gaining entry, timing, collecting artifacts, observing and interviewing, and ethics. The chapter will conclude with a detailed description of the analytical processes applied to the data.

\section{Research Overview}

The Orff-Schulwerk approach has gained much global recognition since it arrived in the United States. Its fundamental approach has demonstrated to be a valuable contribution to developing children's learning in music education. Explicitly, the process of music-making within the Orff-Schulwerk approach has proven to be useful to children's musical experiences and fundamental understanding while stimulating their creative ability and potential (Choksy et al., 2001; Churchley, 1980; Hickey, 2003, 2012; Orff \& Walter, 1963; Shehan, 1986; Thresher, 1964; Wang \& Springer, 2013).

Throughout the Orff-Schulwerk approach, children are continuously encouraged to immerse themselves in "playful musical exploration" and "creative expression" (Beegle \& Bond, 2016) as they navigate through their creative thought processes. The depiction of musical ideas in composition allows children to freely express their musical thinking while demonstrating their conceptual music understanding (Wiggins, 2003). Through the subsequent act of composing and improvising, children may develop their musical identity as they immerse themselves within the compositional process, directly 
enhancing their musical experiences and musicality while inspiring their creative novelty (Hickey, 2012). Considering the divergent-to-convergent process through the stages of preparation, incubation, illumination, verification (Webster, 1990) allows children to display their musically and creatively inclined skills by achieving compositional outcomes. Children with continuous exposure to composition within specific parameters are more likely to develop satisfying musical compositions while inadvertently improving their creativity. Furthermore, children also demonstrate the collective work of their divergent and convergent thought processes when given substantial opportunities to compose musically (Wang \& Springer, 2013).

Despite enhancing children's musicality, musical experience, and creativity, there is an insufficient amount of data examining their creative processes in music composition through the Orff-Schulwerk approach (Hickey, 2003, 2012; Kratus, 1989; Orff \& Walter, 1963; Thresher, 1964; Wang \& Springer, 2013; Webster, 1990; Wiggins, 2003; Womack, 2008). The implementation of composition inspires children's creativity and improvisation, as discussed in multiple sources examined in this study, with a growing area of research on children's creativity using Orff instrumentation during compositional activities (Choksy et al., 2001; Frazee \& Kreuter, 1987; Landis \& Carder, 1972; Springer, 2013; Wang \& Springer, 2013; Womack, 2008). However, further knowledge on musical creativity through the compositional process could contribute to a better understanding of children's creative thought and enhance teaching strategies. Its implementation on children's musical creativity can provide music educators with the opportunity to strengthen their educational approach to composition, further facilitating and nurturing 
children's independent thinking while encouraging their creative authenticity through music-making (Hickey, 2003, 2012).

\section{Central Purpose and Research Questions}

The purpose of this study is to explore the creative processes of elementary school students in music composition through the Orff-Schulwerk approach. Specifically, this study will examine their creative processes through the framework of divergent to convergent thinking within Webster's (1990) model of creative thinking in music. The following central questions were used to guide this study:

1. What is the nature of children's creative processes during an Orff-based music composition task?

2. What are the children's perceptions of their creative processes employed during an Orff-based composition task?

3. What are the teacher's perceptions of teaching and learning music composition through the Orff-Schulwerk approach?

\section{Descriptive Qualitative Research}

Descriptive research study refers to studies that are descriptive in nature, including rich descriptions of experiences and/or perceptions of a particular phenomena, to gain understanding on the phenomena that is rarely and/or poorly understood (Fraenkel, Wallen, \& Hyun, 2012). Descriptive studies usually describe particular phenomena within the healthcare field, but can also be used to provide a descriptive research analysis on the phenomena within other fields outside of the healthcare industry. 
For the present study, a descriptive qualitative study research design was used to explore elementary school students' creative thought processes in music composition within an Orff-based music classroom setting. Characterized by the compositional processes of the approach's implementation, the participants of the student groups within the Orff-based music class will provide an extensive look into the children's creative thought processes to gain greater insight of their musical creativity in music composition. Various data methods were collected to gain an in-depth understanding into the phenomenon within the Orff-based music class. Data was generated through my nonparticipant observations, field notes, focus group interviews with student participants, interview with the teacher participant, audio material, and collected artifacts (i.e., lesson plans, assignments, tasks to complete, compositional products). Upon collecting the data, a descriptive analysis was conducted focusing on certain characteristics of the group participants' developmental stages of the creative processes to better understand children's musical creativity in music composition through the approach's implementation.

\section{Participants and Sampling}

The purpose of this descriptive qualitative study is to explore the creative processes of elementary school students in music composition through the OrffSchulwerk approach. Therefore, to examine the phenomenon of children's musical creativity in music composition through the approach's implementation in-depth, I chose to study one elementary music class, divided into two student groups. This elementary school music class consisted of twenty-two 5th grade students and their teacher to represent the purposive and convenience sample of the population (Fraenkel et al., 2012), 
per the teacher's discretion. For the sake of this study, the teacher was selected based on the criteria that: (1) they possessed a minimum of the Level I certification in the OrffSchulwerk approach and (2) they integrated the approach's implementation into their musical instruction. The Orff-Schulwerk certification program is a "comprehensive threelevel curriculum comprised of pedagogy, recorder, and movement is designed to develop the musicianship, creativity, and teaching strategies of the adult learner" (American OrffSchulwerk Association, n.d., para. 1). The student and teacher participants were chosen from a public charter school located in Miami, FL. The teacher participant had completed all three levels of the Orff-Schulwerk Certification and regularly integrated the approach's implementation into the music program and daily musical instruction.

Ultimately, I chose fifth-grade elementary school students as the participants for my study due to the teacher's discretion. The student and teacher participants were selected from a school in Miami-Dade County with a music teacher who had specific qualifications. The above criteria were necessary to choose the teacher participant for the teacher to instruct the student participants through the compositional activities presented during this study. The two student groups within the one elementary school music class that represent the purposeful and convenient sample of the population were based on the specified criteria that:

1. The Orff-Schulwerk approach would be integrated into their daily musical instruction.

2. The music teacher would incorporate compositional activities through the approach's implementation. 
3. The teacher would encourage the student participants to partake in the musicmaking process using Orff instrumentations to inspire their creative novelty. The above criteria were necessary to select student participants to have students who were familiar with the approach's process and instrumentation to conduct the study promptly. Furthermore, the participants of the focus group were conveniently selected by the teacher participant based on the criteria that: (1) the student participants had returned their consent forms allowing me to proceed with the nature of my study, and (2) the student participants in the focus group were not in the same student groups.

Since children typically develop their musicality and musical experiences through the fundamental elements of music-making and creativity within the Orff-Schulwerk approach (Choksy et al., 2001; Churchley 1980; Hickey, 2003, 2012; Orff \& Walter, 1963; Shehan, 1986; Thresher, 1964; Wang \& Springer, 2013), the students within both student groups of the elementary music class possessed the skills and knowledge gained from the approach to utilize them creatively throughout their musical compositions. This rationale contributed to the foundation for me to examine this specific grade level within the elementary school music class. Most Dade County elementary schools only require educators to teach music to grades 2nd through 5th ("2018-2019 Student Progression Plan," n.d., p. 55), further rationalizing my choice to examine elementary school students of the particular grade level mentioned above.

Recruitment. To recruit for this study, I used my professional networking connections with active Dade County Public School teachers and South Florida Orff Chapter (SFOC) members. An overview of the study was given to my collegial associates, and they were asked if their music class(es) might fit the outlined criteria. I 
provided them with printed and electronic versions of a recruitment letter to pass along to other teachers with music classes that might fit the criteria (see Appendix C). The recruitment letter displayed a brief overview of the study and described what potential participants (i.e., teacher and their music classes) could expect from participating in the study. Additionally, my and my research supervisor's contact information was provided to the school's administrators, teachers, and parents of the children within the participating music classes should any further questions or concerns arise at any point.

Consent and Assent. Prior to study commencement, I obtained written parental consent from the participants' parents or guardians (see Appendix D), written adult consent from the participating music teacher (see Appendix E), written assents from student participants (see Appendix F), and authorizations for recording from the participants and participating music teacher (see Appendix G). The consent process took place in an initial meeting with the participating music teacher, where I could explain the study and answer additional questions regarding the study or research process. Student written assent was initiated afterward through an arranged meeting with each participating music class, which allowed me to explain the study and answer additional questions that the students had. Parental/legal guardian consent forms were also sent home, along with an explanation of the study, requesting parental authorization so that their child(ren) could participate in the study. Furthermore, recording authorization forms were also sent home to request permission to audio record the student participants throughout the study.

Gaining Entry. Descriptive research studies involving children can experience multiple common issues regarding gaining entry through their gatekeepers (i.e., parents 
and teacher). Gatekeepers are referred to as the individuals responsible for granting researchers access to research sites and permitting their research studies to proceed (Creswell, 2014). In this study, I gained access to the student participants' gatekeepers through my professional networking connection with the participating music teacher. The music teacher approached the students in her participating classes with printed recruitment letters (see Appendix C) to take home or email their parents/guardians an electronic version of the recruitment letter. Although circumstances did not allow me to communicate with the student participants' gatekeepers directly, I had the opportunity to meet with the student participants before the study commenced. This opportunity was authorized through an initial meeting arranged by the music teacher with the participating music classes. During this initial meeting, I had the chance to interact with the student participants through informal conversations before data was generated. These informal conversations served as a gentle introduction, which allowed the student participants to become accustomed to my presence within their school environment. Subsequently, student participants could naturally proceed with their daily activities and react to emerging circumstances during the study as I conducted my weekly observations. Towards the end of the study, the selected student participants of the focus group and the teacher were able to interact with me undisturbed; the focus group participants and teacher were able to exercise genuine reactions uninfluenced by my presence, which allowed my interviews to proceed in a natural and easygoing fashion. Furthermore, the music teacher (i.e., gatekeeper) chose the time and place of all observations and interviews to ensure the participants' comfort and natural flow of the data generating process. 


\section{Data Generation}

Time Frame. Following Institutional Review Board (IRB) approval from Florida International University and obtaining the required consent forms and authorizations, data generation lasted six weeks. Within those six weeks, the data generation was divided into two pivotal sections.

The initial 5 weeks of the study allowed me to generate data from the two student groups within the one elementary school music class. These data methods were generated by non-participant observations, field notes, audio material, and artifacts (i.e., lesson plans, assignments, tasks to complete, compositional products). The student groups within the elementary school music class were observed weekly for one hour per week. The final week of the study consisted of semi-structured interviews with one focus group and the music teacher. Interviews were begun with an open-ended question to elicit different perspectives regarding emerging circumstances during the observation phase. Since the selected student participants of the focus group and the teacher could interact with me consistently, they were uninfluenced by my presence. This allowed my interviews to proceed in a natural and easygoing fashion. A continual record of my findings was generated via field notes and audio recordings. Upon generating the data, a descriptive analysis was completed focusing on certain characteristics of the group participants' developmental stages of the creative processes to better understand children's musical creativity in music composition through the approach's implementation.

Observations. Descriptive qualitative research studies investigate the richness of particular circumstances, which are mostly documented through observations and 
interviews. Researchers conducting non-participant observations focus on the circumstantial events occurring in real-time from an outside perspective. Rather than participating in the activity, these researchers act as "observers" of the emerging circumstances from a distance (Fraenkel et al., 2012). Observing through a nonparticipant scope provides researchers insight into the participants' reactions to emerging circumstances during the data generating phase while remaining impartial to these events. Researchers can also better understand the participants within their environments and their reactionary responses to emerging circumstances when using non-participant observations as a data method within their study.

In this study, observations were conducted through a non-participant observational scope. Specifically, these non-participant observations were conducted via an observer-as-participant role, where student participants knew of my inherent role as a researcher while I completed my weekly observations. I ensured that the students could become accustomed to my presence within their school setting and continue with their daily activities as I recorded my observations. The previously described efforts allowed me to observe their natural reactionary responses to emerging circumstances during their creative compositional project activities throughout the study.

Field Notes. Creswell (2014) refers to field notes as the researcher's record of observations of the participants' behaviors and activities witnessed during the study. While I observed in the field, I maintained a record of detailed notes generated from observing the participants within the study. This record of detailed notes described all accounts and observations within my fieldwork and was jotted in a dedicated "field notes" notebook. Reports and observations in my dedicated notebook included informal 
conversations and interactions with participants and group dynamics, conflicts, and atmospheric conditions among designated group members that were present during the observation phase. In addition, non-verbal behaviors of the focus group were documented during the interview phase.

When taking field notes, my dedicated notebook was organized by having a title header for each entry that stated the date, time, and the specific student group observed. A combination of abbreviations and acronyms was used throughout my field notes for efficiency during the observation phase; one hour of observing both student groups within the elementary school music class was insufficient to jot down my entire thoughts and quotes. Additionally, recorded audio files and memorization served me a great deal when returning to specific experiences to fully describe the details when I was restrained with time. Expanding on my notes required me to use my short observational summaries and compile them into full narratives that would be adequately coherent when used during my data analysis. These strategic methods of note-taking essentially helped me with my observations. I found myself more attentive towards characteristics that further needed to be addressed within the study.

Interviews. Within a descriptive qualitative research study, interviews serve as another medium of data generating methods that work in tandem with the recorded observations to verify the generated data (Creswell, 2014). Focus group interviews in descriptive qualitative studies typically seek to perceive participants' thoughts and opinions towards an issue(s) and gather their different perspectives (Freankel et al., 2012). 
In this study, semi-structured interviews with the participating music teacher and one focus group were conducted. The focus group consisted of 5 student participants and were selected by the music teacher; this focus group represented one student participant each from one student group of the elementary school music class. The focus group interview was conducted once at the end of the study and was approximately thirty to forty minutes in duration. Interviews with the teacher consisted of an informal interview during week two of the study that lasted roughly twenty-five minutes. At the end of the study, a formal interview lasted about an hour and a one-hour follow-up interview during data analysis for member checking. Interviews were begun with an open-ended question to elicit different perspectives regarding emerging circumstances during the observation phase. A continual record of my findings was generated via field notes, and documentation of the participants' non-verbal behaviors was recorded in my notebook. In addition, interviews were audio-recorded using a small Olympus digital voice-recording device during the study. Transcriptions were completed following the interviews using oTranscribe.com, a free website that allowed me to slow down and pause the audio recordings to transcribe entire conversations accurately. Data generated by these interviews, along with observational notes, guided my descriptive analysis.

Role of the Researcher. My role as a researcher in this study was influenced by my interest in children's musical creativity within the Orff-Schulwerk approach. Although I have no formal background in teaching music within the classroom, I have had opportunities to interact with children in social contexts via private music lessons that I have taught. In addition, I was formerly employed as an aftercare elementary school teacher for a year before becoming a graduate teaching assistant in 2017 and a graduate 
research assistant in 2019 at Florida International University. Furthermore, I have prior knowledge within the Orff-Schulwerk approach through completing my first level of the Orff courses and receiving my Orff Level I certification during the Summer of 2018 at Florida International University.

While designing this descriptive qualitative study, various scholarly works and research design textbooks were utilized to determine the types of data generation methods used in my study, and how to generate it. Ethical issues regarding children were a great concern to me. To prevent such issues, I met with the class weeks before data generation to explain the study again, answer their questions, and informally interact with them during their regular class time. These efforts allowed the children to become accustomed to my presence within their school setting, and minimize any disruptions. The children were also able to consistently interact with me during their group composition activities, which allowed my interview to proceed in a natural and easygoing fashion as they shared their different perspectives. These experiences, along with my past experiences, gave me opportunities to interact comfortably with the children, and to gain further insight into their behaviors and reactionary responses throughout the study. In addition, I was able to gain an in-depth understanding of the developmental stages in the children's creative process through their music-making in the Orff-Schulwerk approach. The nature of this qualitative study was also predicated on providing rich descriptions on how the musicmaking process within the approach's implementation could be incorporated into promoting children's musical creativity. I hope that the experiences encountered within this study will be beneficial to my own instructional practice one day when I integrate into my career field as a fellow music educator and provide me with the knowledge and 
solace needed to aid my future students' musical creativity in music composition through the approach's implementation.

Ethics. Research involving human participants raises many concerns with ethical issues that may emerge during the study. Therefore, researchers are required to document all anticipated ethical concerns, address the concerns throughout their research, and follow the code of ethics protocol of the human subject review board affiliated with their institution (Creswell, 2014).

For the sake of the present study, I adhered to the policies abided by the Florida International University Institutional Review Board (IRB) to ensure I met the outlined issues of consent. Parents or guardians granted permission for the participants to participate in the study by signing the written consent forms (see Appendix D) and authorization of audio recording (see Appendix G) prior to study commencement. The privacy interests of participants were protected by ensuring that the participants were aware of their voluntary participation in the study and that they may withdraw their consent at any time during the study. In addition, information revealing the participants' identity was not published, and pseudonyms were used to ensure their identities were kept confidential. Furthermore, all generated data was stored on my computer and flash drive dedicated to this study. The contents stored on my laptop and flash drive were encrypted and password-protected to secure the participants' privacy and confidentiality. Both my computer and flash drive were in my possession during the study, and I only used the raw data stored on these items for analytical purposes.

Data Management and Organization. Researchers often develop protocols to record data from their observations and interviews within the field within a qualitative 
research study. These recorded data transform the generated data from the field into organized/coherent narratives that are readily available and useful for the study's data analysis (Creswell, 2014).

In the present study, a wide range of data was generated to illustrate the phenomenon of children's musical creativity through the Orff-Schulwerk approach within the elementary school music class. A data record was constructed around the raw data generated in the field. This record was expanded on my extensive observational and interview field notes, audio materials, and artifacts from the site, transcribed into full narratives, and later coded for descriptive analysis and interpretation purposes. During the data record construction, my annotated and described entries of the generated data were transcribed into full narratives to be readily available and easily identifiable within the data record. These entries were then structured into larger narratives to better understand the two student groups of the elementary school music class within their contexts, becoming significantly useful and coherent during my data analysis.

Constructing a data record to transform the data into organized emerging stages relative to the creative processes also allowed me to fully understand the varying kinds of data generated from my fieldwork. Regardless, the raw data collected throughout the study was retained in my possession and was strictly confidential; the only purpose of the raw data was to be used for my analytical interpretations of the data generated.

\section{Data Analysis}

NVivo. NVivo is a qualitative data analysis software program that I used to organize and analyze my data. This program supports data in the forms of document, spreadsheet, audio, image, and video files, which are encrypted and password protected 
to secure the program's data files. This program also incorporates a collaborative teamwork feature, where collaborating researchers can code, excerpt, and analyze the generated data from a study. Each member of their team has access to the generated data within the program.

Procedure. In this descriptive qualitative study, a data record was constructed surrounding the raw data generated from the field (i.e., observations, interviews, field notes, audio materials, artifacts). The generated data were transcribed in exact detail, providing descriptive and annotated transcriptions of the data generated. The data record was then coded to determine the emerging stages in relation to the creative processes that recurred throughout the generated data from my fieldwork. Through the coding process, I developed descriptions of the participants' creative processes and emerging circumstances within the developmental stages of the creative processes that occurred throughout the music-making process within the Orff-Schulwerk approach. These descriptions were transcribed into narrative passages to further interpret the descriptive analysis findings towards a greater insight into the elementary school music class and two student groups within their contexts. Once the coding process was complete, assuring the findings of my descriptive analysis were valid with regards to the emerging stages that are relative to the creative process provided me with an overall justification for my interpretation of the findings. Throughout the data analysis phase, I retained the generated data in my possession. It remained confidential to protect the participants' privacy interests; the generated data from my data record was only used for my analytical interpretations of the data generated. 
Theoretical Framework. Webster's (1990) work on creative thinking in music was used to inform the data generation, analysis, and organization of this study. The model of creative thinking in music (Webster, 1990) focuses on the interrelationship between multiple components that operate within creative musical thinking. The model begins with a "product intention," advances through the creative thinking process, and subsequently results in a creative product. The core stages that move through divergent to convergent thinking, specifically the stages of preparation, incubation, illumination, and verification, illustrate the creative process's pivotal function that occurs between the product intention and creative product. "Enabled skills" and "enabled conditions" also play a factor within the creative process. These skills are characterized by musical and creative aptitudes, including music, aesthetic sensitivity, cognition, and craftsmanship, while the conditions are characterized by motivation, subconscious imagery, personality, and environment. The final creative product results from the depiction of the thinking process within the enabled skills and conditions.

The purpose of this descriptive qualitative study was to explore the creative processes of elementary school students in music composition in an Orff-based music class. Therefore, I used the theoretical framework of Webster's (1990) model of creative thinking in music to examine the participants' creative processes in music composition through the Orff-Schulwerk approach. Since this study sought to investigate the processes of musical creativity, the core stages that move through divergent-toconvergent thinking, including the stages of preparation, incubation, illumination, verification (Webster, 1990), served as the framework for my data analysis. This theoretical framework helped guide my observations, interviews, and artifact collection to 
learn about the participants' creative thought processes through their music-making. I also interpreted the generated data by focusing on the developmental stages' specific characteristics in the creative process to gain a greater insight into children's musical creativity in music composition through the approach's implementation.

Validation of Analysis. For this study, I used several validation procedures to assure that the emerging stages relative to the creative processes were accurate within my data analysis to gather an overall justification of my interpretation towards the findings. Various data generation methods were used to provide in-depth descriptions of the two student groups to validate my conclusions further. These methods used in the present study included observations, interviews, and collected artifacts to gather the emerging stages within the data analysis and descriptions of participants' creative thought processes through their music-making. In addition, my interpretations of the findings were discussed with my research advisor to clarify preconceptions and avoid influencing the study's outcome. By validating my codified and descriptive findings, I was able to gain greater insight towards children's musical creative thinking in music composition through the Orff-Schulwerk approach. Furthermore, member-checking with the teacher participant also served a part of my validation of analysis towards the codified/descriptive findings, as well as my interpretations of the findings, for ensuring trustworthiness and accuracy of the determined generalized conclusion. 


\section{CHAPTER IV:}

\section{ANALYSIS AND FINDINGS}

This descriptive qualitative study sought to explore elementary school students' creative processes in music composition in an Orff-based music class. The following specific questions guided the study: 1) what is the nature of children's creative processes during an Orff-based music composition task, 2) what are the children's perceptions of their own creative processes employed during an Orff-based composition task, and 3) what are the teacher's perceptions of teaching and learning music composition through the Orff-Schulwerk approach? To examine the phenomenon of children's musical creativity through the approach implementation, I observed one elementary school music class consisting of twenty-two 5th-grade students.

A descriptive qualitative study research design was used to explore elementary school students' creative thought processes in music composition within an Orff-based music classroom setting. Characterized by the compositional processes of the approach's implementation, the participants of the student groups within the Orff-based music class provided an extensive look into the children's creative thought processes to gain greater insight of their musical creativity in music composition. Various data methods were collected to gain an in-depth understanding into the phenomenon within the Orff-based music class. Data was generated through my non-participant observations, field notes, focus group interviews with student participants, interview with the teacher participant, audio material, and collected artifacts (i.e., lesson plans, assignments, tasks to complete, compositional products). Upon collecting the data, a descriptive analysis was conducted focusing on certain characteristics of the group participants' developmental stages of the 
creative processes to better understand children's musical creativity in music composition through the approach's implementation.

This chapter will provide a description and analysis of the participants within the Orff-based music class, including the group participants' developmental stages of their creative compositional process, the student participants' perceptions of their creative compositional process, and the teacher's perception of the creative compositional process. Because the purposeful sampling method I utilized intentionally recruited an elementary music class whose teacher was experienced in the Orff-Schulwerk approach and simultaneously planned to incorporate composition activities, each student group and the teacher presented unique perspectives on the creative compositional process.

The observation data were transcribed, coded, and organized based on the stages that emerged relative to the creative process during data analysis, particularly concerning the divergent-to-convergent stages of preparation, incubation, illumination, and verification. The descriptive analysis also presented indications of enabling conditions, environmental influences, and enabling skills inherent within the specific stages. Interpretations were made based on the interviews and field note data, and are organized based on findings from various perspectives: observations of the two student groups actively engaged in the compositional tasks, the student's own perspective, and those of the teacher. Field notes served as the primary data for the first research question; interviews and member checking were used to validate and provide further context to the field notes. For the second and third research questions, interviews served as the primary data, while field notes and member checking were used for validation and contextualization purposes. 


\section{Creative Compositional Process}

Annie's Group. Annie's group consists of three amicable, bright, and virtuoso girls named Annie, Nicole, and Susana. Annie is a witty and candid adolescent girl with long dark brown hair who naturally gravitates towards leadership roles with her confident attributes. Nicole is an amusing, curious, and inventive adolescent girl with long black hair, but is also notorious for commandeering task cohesion within the group. Susana is a recognizably shy yet easy-going adolescent girl with medium length brown hair and glasses who possesses adaptive qualities to incongruous situations within her group.

Bassline. As Ms. Smith introduced the new composition project to the class that groups would be creating throughout the next five weeks, Annie's group dynamic was no different than the rest of their class; observant yet galvanized and intrigued by this new project on which they were about to embark. However, the girls' faces, especially Annie's, which turned pale, were distinctly noticeable when Ms. Smith mentioned that this project would be "more involved" than the last one: a simple "one line of music" composition. Nevertheless, the girls seemed to be ready for the tasks that the project would require them to do.

Ms. Smith elaborated on the "more involved" composition project, stating that it would consist of three elements (i.e., a bassline, a melody, and a color part) to form the extensive multi-layered composition project. She began by briefly describing each component before outlining the first element (i.e., bassline) of the project. When describing the specific details of the bassline, Ms. Smith mentioned that each group would have to use a short ostinato of simple rhythms, as well as pick one of three borduns (i.e., chord bordun, broken bordun, or crossover) to use with the ostinato. She then 
explained that the bassline should be "short but interesting, simple but original" and then wrote an example of a bassline on the board; a quarter note, a quarter note, a set of eighth notes, and lastly a quarter note. She went on to describe the different borduns and placing specific parameters when groups would use each bordun on the xylophones, such as playing the keys of $\mathrm{C}$ and $\mathrm{G}$ together when using the chord bordun, playing the keys of $\mathrm{C}$ and $\mathrm{G}$ separately when using the broken bordun and only using the "big $\mathrm{C}$ and big G" keys, and starting to play on low $\mathrm{C}$ to low $\mathrm{G}$ and crossing over to play high $\mathrm{C}$ when using the crossover. As Ms. Smith continued to outline in detail the different borduns that groups could utilize for their basslines, I had noticed that Annie and Nicole were already discussing which kind of bordun they should use for their bassline. Annie suggested, "Maybe crossover." Nicole agreed, "I think we should do crossover."

Once Ms. Smith had outlined the task at hand, she provided the groups with twominutes to "experiment" the different borduns and decide which bordun their group would use for their composition project. During these two-minutes of experimentation, Annie had experimented with the other two borduns but mentioned that those were too difficult to play through. Continuing exploring with the crossover bordun, Nicole said to her group, "Okay, so listen," and played a crossover bordun with the rhythmic pattern of a quarter note, a set of eighth notes, and another quarter note. Annie responded, "Let me hear it again... okay, so it will be Ta, Ti-Ti, Ta... you didn't cross, you got to cross."

At that point, I was intrigued to know what the group was thinking about doing for their bassline and what rhythm they would choose to use; more so, I was curious to know if the group would consider keeping Nicole's exploration on the crossover bordun with the specific played rhythmic pattern as part of their bassline. Nicole reiterated, "I 
was thinking Ta, Ti-Ti, Ta..." "Were doing crossover, for sure," Annie said. "Oh yeah," Susana echoed. Annie confirmed, "Yeah, crossover for sure. Ta, Ti-Ti, Ta, so... yeah. It's pretty good. But now me... let me try,"

The group had already decided that Nicole's exploration would benefit their bassline when Ms. Smith gave the groups another five-minutes to focus on the specific bordun that interested them during the first two-minutes of experimenting with the different borduns. However, Annie had thought of and played her exploration, but Nicole stated that "it was too complicated," so the group kept Nicole's exploration instead. Nicole's opinion towards Annie's exploration was the turning point of the group's dynamic, which became more perceptible as the study continued its course.

I wanted to know how long they wanted to make their bassline. While the group continued to play through the crossover "Ta, Ti-Ti, Ta" pattern that originated from Nicole's exploration, Nicole excitedly asked, "can I do two measures?... We can do..." and played her initial crossover rhythm in reverse. After she witnessed a group exploring with a chord and broken bordun combination for their bassline, Ms. Smith emphasized to the class that groups could combine more than one bordun. Once again, Nicole's curiosity sparked, and mentioned that the group should also have a combined bordun for their bassline. However, Susana was well ahead of her and played Nicole's crossover rhythm and added a chord bordun on two quarter notes (i.e., high $\mathrm{C}$ and low $\mathrm{G}$ played together, then low $\mathrm{G}$ and low $\mathrm{C}$ played together). Annie and Nicole seemed to have also liked Susana's exploration with the chord bordun, which led the group to continue working through the crossover and chord bordun combo to determine what exactly would be adequate for their bassline, specifically the rhythmic patterns they would use for their 
combined bordun ostinato. The girls were rehearsing their collaborative bordun bassline when Ms. Smith called upon their group to play their bassline for her during the final minute of class.

Throughout this compositional activity, three of the four stages relative to the creative processes emerged: preparation, illumination, and verification. It was evident that each of the three girls' creative compositional processes did not progress linearly, but instead in an interchangeably cyclical manner. Their preparation stage was the first apparent creative process. They began the bassline compositional activity where they were planning out which borduns they were going to explore and exploring with different rhythmic patterns for their ostinato. This stage was very fast-paced and was the least amount of time spent throughout Annie's group, possibly stagnating the group's potential to produce an even more creative bassline. However, their illumination and verification stages overlapped by interacting between and among each other, which Wiggins (2003) elaborates on in her model called the frame. Both the illumination and verification stages were the stages that the girls spent the most time on as they revised, edited, and formed new ideas for their bassline and then rehearsed and polished the ostinato for a successful execution at the end of class. The group's creative process for this compositional activity traveled on a spectrum between the stages of preparation, illumination, and verification, and coincides with Landis and Carter's (1972) study in which they elaborate that the subsequent act of improvisation on Orff-based instruments encourages students to use compositional devices such as borduns, ostinatos, and rondos that are apparent specifically within the Orff-Schulwerk approach. 
Melody. During the third week of the study Ms. Smith would articulate that the class work would involve groups revising, fixing, and learning to play their 'A' section melodies. While this might have been an exciting experience for the group to consider, it was far from it. In the previous class, the group had begun and finished creating their ' $A$ ' section melody, but unfortunately, Nicole had not turned in the group's melody sheet, which resulted in a grade of ' $F$ ' for the last class. Ms. Smith prompted her to go to her homeroom teacher's classroom to find and bring her group's melody sheet so that they could work on the objective of the day.

However, Nicole did not have the group's melody sheet in her bookbag, prompting Ms. Smith to aid the group so that they could catch up to the rest of the class. I witnessed how the dynamic of this group shifted completely from the first week of the study. Before, the dynamic was already rocky due to Nicole's dismissal of Annie's exploration because she thought it was "too complicated." Now, both Annie's disdain for Nicole intensified due to the missing melody sheet, and neither she nor Susana wanted to collaborate further with Nicole.

Ms. Smith scaffolded the group's new exploration process for their melody, guiding them measure by measure so that they quickly got to revising and learning their melody. Ms. Smith asked each group member to come up with a rhythmic pattern for the measure they were assigned to do and reminded them of the appropriate rhythm options (See Figure 4.1).

After the girls had written down their rhythmic patterns for the four measures of their A section melody, Ms. Smith again scaffolded the group's "exploration" to guide them with the pitches that they would choose for each measure, but provided specific 


\section{Figure 4.1.}

Melody Rhythm Options.

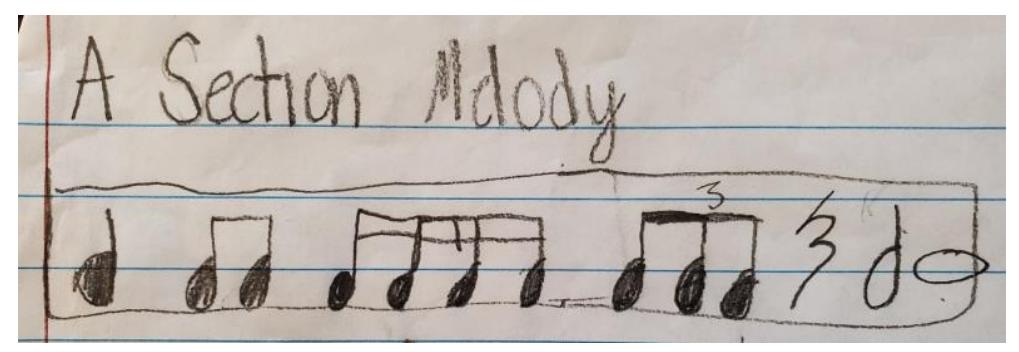

parameters with regards to selecting pitches for their melody; "You have to start on C, you have to end on $\mathrm{C}$. Write me the letters that go in between." Upon adding the pitches to their new 'A' section melody, Ms. Smith addressed the entire class again to instruct them that each group would have to read their melodic rhythm out loud to her. Reading the rhythm was an easy task for the girls since they had already spoken through their melodic rhythm fluently with Ms. Smith. She then instructed them to go on to the next task, speaking the melodic rhythm on pitches. However, this caused another group conflict between Annie and Nicole: "G-G... E-E," Annie chanted. "But you are doing this, but it's not that fast," Nicole argued. "Yes, it is," Annie snapped. Susana then mimicked Annie in solidarity, "E-E." Annie responded in affirmation, "E-E, that's what I did." Nicole protested again, "But the rhythm, though!” Susana growled, "Nicole, just forget about her!"

Besides experiencing several unpleasant moments among themselves, the girls were having difficulty speaking the pitches in the third measure (i.e., the "conflict" measure) of their melody. However, they had no problem speaking it rhythmically. Specifically, the group had trouble transitioning from the triplet pitches to the half note pitch, which could be described as a tongue-twister (See Figure 4.2). In addition to 
having trouble speaking through the melodic rhythm on pitches, both Susana and Nicole would sing through or skip the rest in Another issue that arose within the group was whether or not they should keep the silence during the rest or if they should speak the word "rest" or make the shushing sound. "I'm just saying how it should be no sound," Susana said. Nicole chanted her rhythm and then responded, "C-C, A-A-G, A--. I know, but we're saying it, we're saying the letter." "Well, there is no letter on it," Susana argued. "But we still have to say it," Nicole maintained. Susana insisted, "No, we don't... That's how it is in real music."

As the group progressed to the third task of that day, the girls had some problems playing through the melodic rhythm of their melody, again specifically with skipping or singing through the rest in measure two and with the transition of the pitches in the triplet to the half note in measure three. However, most of the time, Susana played the triplet in measure three as a set of eighth notes, which Annie helped her play the triplet in one beat by speaking the melodic rhythm on pitches while she played through the melody. Meanwhile, Nicole would mostly skip through the rest of measure two, annoying Annie to her core and making her yell "rest" several times. Additionally, while Susana was learning to play the melody on the xylophone, she elaborated that an easier transition to play the whole note in measure four would be to have that whole note played on the high C key rather than the low C key (See Figure 4.2).

Throughout this compositional activity, one stage relative to the creative processes emerged: the verification stage. However, the group did experience going through a "preparation" and "illumination" stage as an "artificial creative process" with the teacher's scaffolding that provided guidance, structure, and parameters towards the 


\section{Figure 4.2.}

Melody of Annie's Group

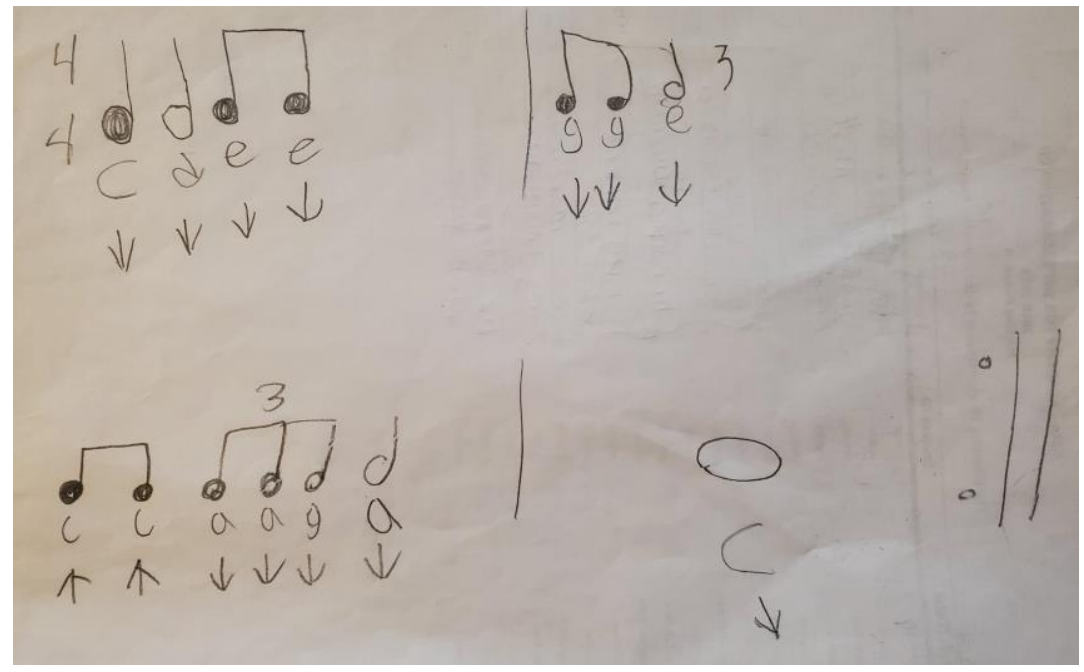

group's "exploration" and "refining" of their melody. Specifically, Ms. Smith guided Annie's group through their "preparation" stage, constituting as an "illumination" stage as the group "created" their melody as she walked them through the rhythmic patterns and pitches appropriate for them to use in each measure. In addition, indications of enabling conditions and environmental influences were inherent within these specific artificial stages. More specifically, the group experienced several conflicts among themselves, particularly among Nicole and her peers, resulting in a negative influence on the group's motivation within their environment, which impacted their creative novelty, craftsmanship, and aesthetic sensitivity, all of which are an integral part of Webster's (1990/2002) model of creative thinking in music. These indicators of enabling conditions and enabling skills may have potentially stagnated each group member's production towards creatively producing this compositional activity, possibly resulting in a lack of enjoyment of each group member. 
Bassline and Melody. The fourth week of the study brings a new challenge to each group: combining their basslines with their melodies. Ms. Smith was aware that this would be quite difficult for her students, which is why she suggested that there be someone on each of the parts reading it out loud for those that would play each part, though with Annie's group having three members in it her suggestion was not that favorable for them. Conflicts were still arising among the girls, specifically the interaction and collaboration between Nicole with Annie and Susana. Nevertheless, at that point, both Annie and Susana were trying to get through the composition project as cordially as possible, doing what they had to do and avoiding any arguments with Nicole unless utterly necessary. However, as the group practiced their bassline, Annie could not sight-read the bassline and proceeded in yet another conflict between the girls (See Figure 4.3). "Isn't it a C?" Annie asked. "It's a rest," Susana responded. Nicole looked at the paper and confirmed, "Oh, that's a rest." Annie disagreed, "No, that's not a rest because you have a Ta under it." Nicole explained in frustration, "It's probably there, but we don't speak it."

Ms. Smith walked over to the girls to hear them play their bassline but quickly noticed they had written their bassline in $\frac{3}{4}$ instead of $\frac{4}{4}$, and advised them to correct the mistake by adding a rest to each measure of their ostinato. However, Nicole wanted to constantly change the bassline to "spice it up" even though Ms. Smith had helped them correct their bassline with the addition of the rests, which made Annie and Susana abundantly irritated with her audacity.

While Annie and Nicole continued to tackle the bassline, Susana attempted to play through the melody but had a difficult time playing it. As Susana continued to 


\section{Figure 4.3.}

\section{Bassline of Annie’s Group}

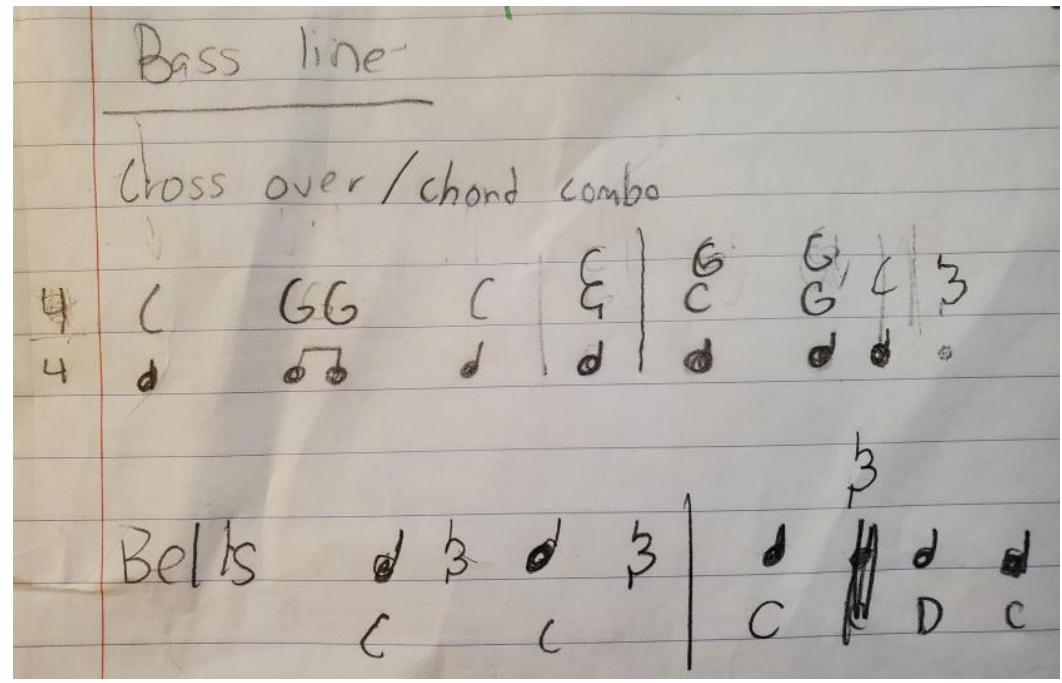

play through the melody but had a difficult time playing it. As Susana continued to practice, she kept struggling to play through the entire melody, but more so when playing the triplet in measure three on one beat. Annie was a huge help to Susana when she was practicing the melody, periodically reminding Susana about the triplet having to be played on one beat and how the group had collectively agreed on playing the last beat of the melody on the low $\mathrm{C}$ key. She was also consistently helping Nicole with the bassline, reminding her of the ostinato's added rests. Even having helped both Nicole and Susana practice playing through two uniquely different elements of their composition project, Annie felt the need to comment about her audition and the requirement to sight-read during this audition where she elaborated on lacking sight-reading abilities.

Upon the group combining both their bassline and melody to perform for Ms. Smith towards the end of class, it became apparent that Susana, with the melody, and Nicole, with the bassline were not playing in sync, driving Annie humoredly mad. 
Nevertheless, Ms. Smith came to their aid and stated that "she [Susana] has: Ti-Ti, Triplet, To-ah against your [Nicole] Ta, Ta, Ta, Rest" and reminded Susana of the half note as well as reminding Nicole to go slow for Susana. Ms. Smith also recommended that the girls speak out loud each part's rhythm before attempting to play through both parts simultaneously. By the end of class, both Susana and Nicole successfully executed playing through the melody and bassline conjointly. Mrs. Smith was very impressed with their performance, as she was for the rest of the groups.

Two stages of the creative processes emerged in this subsection, which were illumination and verification. As mentioned in the earlier subsection, Annie's group did experience an "artificial creative process" of the preparation and illumination stage since the teacher scaffolded their "exploration" and "refining" of their melody by guiding, structuring, and having parameters for their "creating the melody." However, particularly within this subsection, the group traveled on a spectrum between their illumination and verification stages; illumination as the group had to edit their bassline due to writing it in $\frac{3}{4}$ instead of $\frac{4}{4}$, which Ms. Smith assisted them by advising to add a rest at the end of each measure in their ostinato, and verification as the girls had to rehearse and polish each part (i.e., the bassline and the melody) before combining and playing both parts simultaneously.

Additionally, indications of enabling conditions and environmental influences were inherent within these specific stages as conflicts continued to arise between Nicole and her peers when she wanted to change the bassline even after the teacher helped them fix it to be in a $\frac{4}{4}$ time signature. This conflict may not have had a great influence on the 
group at that moment. However, previous conflicts did affect the group's overall motivation within their environment, which had impacted their overall creative novelty, craftsmanship, and aesthetic sensitivity. Furthermore, enabling skills, such as conceptual understanding regarding rhythmic and formal notation, were inherent of playing the bassline and melody simultaneously. Although Frazee and Kreuter (1987) state that Orffbased instruments can facilitate music-making activities through sound recognition while reinforcing rhythmic coding, such was not always the case with Annie's group since their conceptual understanding of rhythmic and formal notation did lack when it came to sightreading the bassline and melody, but more so with the notation in the melody since their third measure included more complex rhythmic patterns.

Color Part. During the final week, Ms. Smith asked Annie's group to play both their bassline and melody parts simultaneously so that she could provide feedback before they added the final element of their compositional project, the color part. While observing another group, I overheard Ms. Smith mention that "the bells [melody] felt a bit off" as Susana played through the rest or did not hold the half note for its total note value. However, I was under the impression that the girls would have gone with the shakers or the triangle for their color part instead of the bell since they were contemplating between the two unpitched percussion instruments at the beginning of class.

When Ms. Smith called upon their group again to hear what they had done for their color part, Nicole played the little bell quite frequently throughout the composition as Susana played the melody. Annie played the bassline "To me, the little [melody] bell is overpowering the melody," expressed Ms. Smith to the group. Ms. Smith then 
recommended that Nicole switch mallets so that the little melody bell would not be louder than the melody or to play the little melody bell less frequently throughout the composition. It was apparent that the girls had some thinking and revising to do for their color part before the final performance, which was quickly approaching.

As the end of the class neared, groups took turns performing their extensive multilayered composition project to Ms. Smith. When Ms. Smith called upon Annie's group, Susana played the melody, Annie played the bassline, and Nicole played the color part on the little melody bell, and each part was in sync with the other. Specifically, regarding the color part, Nicole created a color part that embellished the bassline and melody divinely, which Ms. Smith described as “playing their color part whenever Annie's part had a rest. So they were filling in a blank space. Whenever Nicole heard the rest, she would add her color part there." However, the formal notation of their color part was illustrated differently than "filling in a blank space" to the bassline (See Figure 4.3).

Three of the four stages relative to the creative processes emerged: preparation, illumination, and verification. Specifically, concerning the color part, the preparation, illumination, and verification stage overlapped with one another given the nature of the color part being formally introduced roughly 15-20 minutes within a 50 minute block period before playing all three elements of their extensive multi-layered composition to the teacher. As in Wiggins (2003) model named the frame, the various characteristics of composing, such as organizing, evaluating, revising, refining, and rehearsing musical ideas, interact between and among themselves, which aligns with my finding on the stages of preparation, illumination, and verification for the color part overlapped with one another that was evident in Annie's group. 
In addition, enabling skills, such as conceptual understanding regarding rhythmic and formal notation, were inherent within the context of creating the color part. Although Nicole wrote down the color part she was to play during the group's final performance, she did not sight-read her formal notation of the color part. Instead, she happened to play on every rest in the bassline ostinato. This switch could result from limited time to successfully progress through each of the creative process stages inherent to Webster's (1990/2002) model of creative thinking in music. In the following chapter, I speak about certain limitations apparent throughout this study, and provide implications for a more conducive approach to implementing creative compositional processes to enrich children's musical creativity in music-making.

Jane's Group. Jane's group consisted of two amicable boys named Aaron and Shawn, and two delightful girls named Jane and Ashley. Aaron is a black-haired adolescent boy who has an energetic and upbeat spirit. Although he is short in stature, his curiosity for music peaks ever so high, which contributes to his group. On the other hand, Shawn is a thorough and determined boy with dark brown hair who seems to be hardworking and ambitious when it comes to executing a task; he also possesses leadership traits that continuously benefitted his group through the composition project. Jane is a quiet yet cheerful and bright adolescent girl with black coily hair who is surprisingly witty from time to time, keeping things within the group lighthearted. Ashley can be described as a dirty blonde, chatty long-haired girl who brings a unique and humorous ambiance to the group and frequently makes her group members laugh.

Bassline. It is the first week of the study, and we were nearly at the end of class for the day. Ms. Smith had just asked each group to play their exploration of the 
bordun(s) they decided on for their bassline ostinatos. When Ms. Smith asked Jane's group to play their exploration, Ashley played a combination of a chord and broken bordun ostinato, with the chord bordun part at the beginning of their ostinato on two quarter notes playing the low $\mathrm{C}$ key and the low G key together. The broken bordun part towards the end of their ostinato on a set of eighth notes played on the low G key to the low $\mathrm{C}$ key, followed by a quarter note on the high $\mathrm{C}$ key. However, the group had formally notated their combined bordun ostinato as the chord bordun part being low $\mathrm{G}$ key, and high $\mathrm{C}$ key played together. The broken bordun part being played as low G key to high C key followed by low C key (See Figure 4.4).

\section{Figure 4.4.}

Bassline of Jane's Group

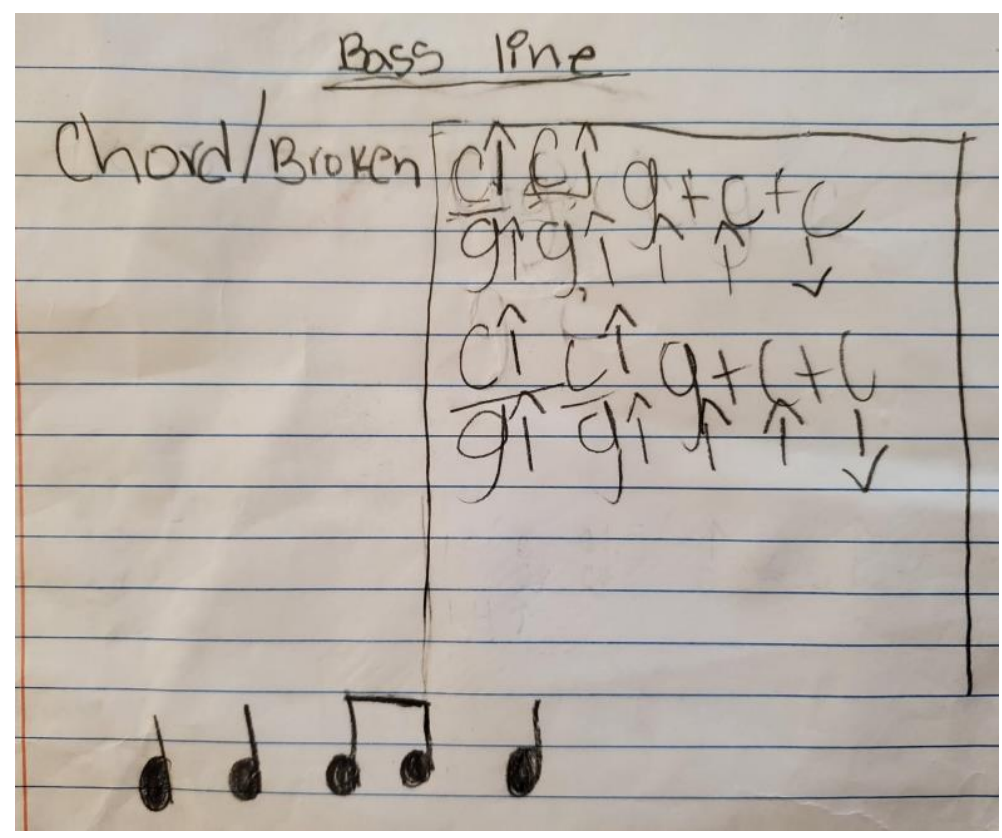

Ms. Smith liked the group's chord and broken bordun combination. She reminded them to add arrows to the different $\mathrm{C}$ and $\mathrm{G}$ keys they were using (i.e., an arrow pointing up for any high pitch and an arrow pointing down for any low pitch). She also asked the 
group if they had written down the rhythm they had just played for their bassline, but they had already notated it. Even though the group had played their bassline somewhat differently in terms of the pitches they notated, rhythmically speaking the notation was accurate to the exploration they had just played for Ms. Smith.

Three of four stages relative to the creative processes were speculated to have emerged: preparation, illumination, and verification. Although I was unable to witness these stages emerge particularly in Jane's group during this compositional activity, I was fortunate enough to have observed their exploration of the borduns chosen for their bassline ostinatos towards the end of class. The navigation of their creative compositional processes was not abundantly clear on its function throughout this compositional activity. Still, given my observation of Annie's group during this exact compositional activity and my member checking of the findings with Ms. Smith during our interview, I speculated that, like Annie's group, the stages of preparation, illumination, and verification were inherent, with the verification stage being the specific stage I witnessed Jane's group having gone through towards the end of class.

The group had played a bassline that was slightly different pitch-wise to what they had notated on their bassline sheet, although rhythmically speaking, the notation was accurate to what they had just played for Ms. Smith towards the end of class; this was also the case when they played their bassline simultaneously with their melody for their final performance in week five of the study, where they had played a different pitch-wise bassline but rhythmically accurate to the notation on their bassline sheet. It was apparent that, when having to sight-read the pitches of their bassline, Jane's group played a reversed version of the pitches notated, thus displaying a lack of reading ability in formal 
notation with regards to pitch accuracy. However, with regards to rhythm accuracy, their formal notation was accurate (See Figure 4.4). This could potentially result from having learned to play their bassline via rote more than via note while going through the stages of preparation, illumination, and verification. In Webster's (1990/2002) model of creative thinking in music, he states that enabling skills and enabling conditions influence the creative processes that move from divergent-to-convergent thinking.

Nonetheless, my speculation was not that their creative processes during this compositional activity were entirely influenced by a lack of musical aptitude or conceptual understanding as outlined in Webster's (1990/2002) model of creative thinking in music. Moreover, my belief is highly predicated upon a contributing fact that the group explored, revised, and rehearsed on an Orff-based instrument (Springer, 2013) specifically, using compositional devices such as different borduns and ostinatos on this pitched Orff instrument (Landis \& Carder, 1972). This most likely facilitated their musicmaking by allowing them to easily recognize familiar sounds while simultaneously reinforcing their rhythmic coding towards their exploration (Frazee \& Kreuter, 1987). However, it was most likely lost in translation when having to notate the pitches they were using for their bassline.

Melody. In the second week of the project, Ms. Smith began like any other regular day; she went through her daily rounds of warm-ups with her class. The class began by singing the "A' section of their "Welcome Song" (i.e., Sol-ri-da) that incorporates simultaneous singing and hand movements. Upon singing through the 'A' section once, Ms. Smith quickly taught the class the 'B' section to their "Welcome Song" that also incorporates simultaneous singing and hand movements. After the class had learned the 
'B' section, Ms. Smith had them sing through the "Welcome Song" once again, only this time singing twice through the ' $\mathrm{A}$ ' section followed by singing twice through the ' $\mathrm{B}$ ' section with both parts gestured through their respective hand movements as the students sang through both parts. She continued her warm-up by transitioning to her rhythmical call-and-response warm-up that consists of clapping and patting in duple and triple meter. She "calls" different rhythm syllables to which the students imitate her as their "responses." Ms. Smith proceeded with her warm-up drills by singing different melodic rhythms using solfege and Curwen hand signs representing each of the pitches sung, where the class responded to her using solfege and Curwen hand signs, as well. She transitioned once more by singing a $\mathrm{C}$ major scale with her students using solfege (i.e., Do, Re, Mi, Fa, Sol, La, Ti, Do) and Curwen hand signs, and then divided the class into two sections. Ms. Smith concluded her warm-up by having one group begin to sing the C major scale using solfege and Curwen hand signs. When that group got to the third degree of the scale (i.e., Mi), the other group began to sing the scale creating a harmonization between both sections. Once the second group arrived at the end of the scale, both sections switched parts so that the other group got to harmonize with their counter-group when they heard them sing the third degree of the $\mathrm{C}$ major scale.

As the class took a seat on the floor of Ms. Smith's portable music classroom, she began to articulate that the day's task will be for groups to begin composing their ' $\mathrm{A}$ ' section melodies. She began by drawing a parallel to the "Welcome Song" the class had just sung through:

So you guys noticed that in our welcome song we had an ' $A$ ' section and a ' $B$ ' section, yes? Yeah. And you were able to repeat the 'A' section and repeat the 'B' section. So, the form, the order of our welcome song was AABB. The final order 
of the songs that we're composing are going to be ABA forms, which means that your 'A' section will be performed twice and your 'B' section will be sandwiched in the middle. Our goal for today, is to try to complete our ' $A$ ' section, and we'll be able to make edits on the ' $A$ ' section later on if we figure out that we don't like something that we did.

Ms. Smith continued by describing that each group's bassline had to be a simple bordun ostinato purposefully to allow the melody to be "more involved" which meant bringing out the melody as much as possible. She further elaborated that the melody would include more complex rhythms than the bassline. She outlined the appropriate rhythmic patterns the groups could use for their melodies (See Figure 4.1) but emphasized that certain rhythmic patterns would be most appropriately placed in specific areas of their melody:

You can definitely use quarter notes, eighth note pairs are great, the occasional set of 16 th notes would be nice to use. The occasional set of eight note triplets would also work nicely... Triplets are super awesome, but have you ever heard a song that just had so much coming at you at once, that you're like, "This is going to get hard." Yeah. So, we want to leave room for our audience to mentally breathe while they listen to our music. Which is why I would encourage you to use the occasional quarter rest, the occasional half note, and even the occasional whole note. Where do you hear the whole notes, where do you usually have like the longest note of the phrase?... Yeah. Julie said at the end, I totally agree. That usually signifies to an audience that that's like the end of the phrase or the end of the song. So I agree, be careful where you use a whole note, is what I'm trying to get at. 
Ms. Smith proceeded to describe the improvisational process that the class had used to explore using the $\mathrm{C}$ pentatonic scale a few weeks ago. She continued by elaborating that the $\mathrm{C}$ pentatonic scale is slightly different from their warm up solfege scale of C major:

The scale in $\mathrm{C}$ pentatonic is kind of like the scale that we sing together: (singing) Do, Re, Mi, Fa, Sol, La, Ti, Do, but it's missing two notes. The pentatonic scale takes away Fa and Ti. So really, all that we have is: (singing) Do, Re, Mi, Sol, La, Do. That's what we're going to be allowed to use. We're taking out the F's, and we're taking out the B's. So you're going to notice that your instrument was missing F's and B's, that was for a reason, because we won't be using them... No burgers, no fries.

After describing the form of the melody, the appropriateness of rhythms in certain areas of the melody, and the function of the $\mathrm{C}$ pentatonic scale, Ms. Smith instructed the groups that the first five minutes of this compositional activity would be spent on the improvisational process before beginning their melodic composition, allowing each group the opportunity to explore through the different sounds and rhythms that appeal to them on the xylophones. She also provided parameters for the groups to follow while going through their improvisational process, such as the groups could not write down anything during this process and their starting and ending pitch must be the key of $\mathrm{C}$ because " $\mathrm{C}$ is Do, that's like our home... we start the day at home and we end the day at home... the high C can be like your vacation home."

Jane immediately began exploring on the xylophone and played a rhythm that included a quarter note, quarter note, a set of eighth notes, and a quarter note. However, her exploration was played on the keys of $\mathrm{C}$ and $\mathrm{G}$ only, so I reminded the group that they could use any pitch in the $\mathrm{C}$ pentatonic scale for this compositional activity. I also elaborated that it was their choice to either use certain pitches or use all of the pitches in 
the $\mathrm{C}$ pentatonic scale, to which Ashley responded that she would like to go "Black Panther wild" with the pitches, then laughed. As the group continued taking turns exploring with different pitches and rhythms on the xylophone, Jane took the mallet and jokingly said, "wait look, C, C... I started on C and I ended on C" which made Ashley laugh. When it was Ashley's turn again, she played her exploration like everyone else had done. It seemed that the group liked her exploration, thus sparking my curiosity as to why Ashley was so fond of these pitches and rhythm pattern was the same rhythm played by Jane. "I think it's easier to memorize it," Ashley said to me, and I responded by asking the group if they preferred to play something easier or harder. Shawn replied, "I mean, it could be harder if it sounds better, but..." Ashley clarified, "Yeah, you see, we'd rather have it harder and sound better than have it easier and like sound..." "...like, simple," Shawn interjected. “...bad,” Ashley retorted.

Ms. Smith asked everyone to get into "rest position," which meant that the class had to stop what they were doing to direct their attention to her. At this point, Ms. Smith would formally introduce the melody by metaphorically comparing the melody to a novel or story. She began by mentioning that, at the beginning of a story or during the first few chapters of a novel, there is a background introduction of the characters being incorporated into the setting. Ms. Smith further explained that the group's first two measures of their melody should be considered the "first two chapters of a novel" where groups inaugurate their song to the audience "without getting too wild or crazy." She clarified that, just like in the improvisational process they had just done, the melody should begin on C's key because "that's home to us" but that groups should be opened to instituting other pitches to their measures Ms. Smith also elaborated that the first two 
measures should be simple rhythms, such as the quarter note, the half note, and the set of eighth notes, and advised groups to leave enough space in measures one and two for the third measure to include the more complex rhythms. She then played and wrote an example of the first two measures of a made-up melody and ended on an E key in the second measure, to which she explained that she ended on an E because "we're not at the ending yet; we're still at the introduction." After demonstrating and writing out her example, she gave the class two-minutes to compose their first two measures.

Jane mentioned that she and her group had just done what Ms. Smith instructed them to do so, but Shawn reminded her that they only had four notes at the moment. Shawn, Aaron, and Jane continued to explore with different pitches, but they seemed to have gravitated towards Jane's initial rhythm of "Ta, Ta, Ti-Ti, Ta" to use for their first measure (i.e., quarter note, quarter note, a set of eighth notes, quarter note). Aaron then played his exploration for the first measure, which followed the simple "Ta, Ta, Ti-Ti, Ta" rhythm, and the group seemed to like it. Shawn further emphasized that the rhythm for measure one would be "quarter note, quarter note, eighth note, quarter note" after Aaron played his exploration. Then he asked Jane to write down the rhythm for their first measure but, by that time, Ms. Smith asked the groups to get into "rest position" once again.

Ms. Smith asked the groups to stop working on the first two measures and direct their attention to her. She reiterated that the first two measures of the melody were the introduction chapters of a story or novel, and then she expressed that measure three is akin to the "conflict of the story" where interesting things happen in the story. Her final metaphorical comparison was that the melody's final measure had to be calm and relaxing 
because it is the measure that "solves the problem," which proceeds after the conflict of the story or better known as measure three. Ms. Smith further explained that for measure three, groups had to use complex or "spicier" rhythms that include sets of sixteenth-notes and sets of eighth-note triplets but could also choose to include simpler rhythms alongside the complex rhythms. She also added that groups "can choose to stay on the same note for a fast rhythm [on the triplets] or... can choose to move to different pitches there" but informed them that moving through different pitches on triplets could be quite difficult although it would ultimately be up to their discretion. The last measure "is going to be like [a] mini-resolution," said Ms. Smith, and then added that the final measure should include simple, yet spacious rhythms such as half notes and whole notes but could also include occasional quarter notes. After she described the appropriate rhythms for both measures three and four using her melodic metaphor, Ms. Smith gave the groups five-minutes to explore and create their measures three and four of their melody.

Aaron quickly began to explore different complex rhythms and pitches for the third measure of his group's melody. "Wait, what about sixteenth notes; Tiki-Tiki, it's one beat. Let's do A-A-A-A," said Aaron, to which Shawn added, "Okay, then let's do: C-C-C-C." However, Jane commented that she was unsure if the group should repeat these sets of sixteenth notes on the same pitches, which made the group further explore the sixteen note pairs on different pitches. "C-C-C-D. Then C-D-C-D," Aaron demonstrated. "I feel like it's too fast," Shawn complained. "It's not," Aaron said. Shawn added, musingly, "But I also feel like it's too simple." "C, E, G, C," Aaron demonstrated again. "Wait, for the third one?" Shawn asked. Aaron explained, "Yeah, for the third one: C, E, G, C. But it has to be complex. 
Shawn continued exploring through various pitches and rhythms while reminding his group that the melody required four beats per measure. "Now we're missing a beat," said Aaron, but in actuality, the group was missing three beats for measure three being that they only explored one set of sixteenth notes since they did not want to repeat the same pitches for the second set of sixteenth notes. I asked him how many beats were they missing if they had just explored through one set of sixteenth notes. However, the group had a bit of difficulty answering my question. Shawn answered, "One beat." "No, TikiTiki is one beat, right?" I asked. Ashley confirmed, "That's one beat, yeah." "So how many beats are you left with?, I asked. Shawn answered, "Three."

Near the end of class, Jane's group was still contemplating what they would do for their third measure. Almost entirely dismissing the sets of sixteenth notes that had just been explored for their third measure, Ashley mentioned how they should have "a dotted quarter note and then a half note," to which Shawn added "just one beat, then To-ah." To help remind Jane's group about the complex rhythms that they had to use for measure three, I asked them to think back on what Ms. Smith had mentioned about the third measure of their melody in hopes that it would help them remember the rhythms they could use. "Wait, I got an idea," said Ashley as she began playing a new exploration. After hearing Ashley's exploration, Shawn advised her that if she desired to use a more complex rhythm with various pitches she should split up the explored complex rhythms to include the pitches she wanted in the rhythmic pattern she was exploring on. "Take one minute to wrap up whatever your thought was and then we've got to pack up," announced Ms. Smith, which Jane's group did their best to write down the rhythm and pitches for their melody quickly. Unfortunately, they could only jot down some of what they 
explored. Jane's initial rhythmic pattern of "Ta, Ta, Ti-Ti, Ta" (i.e., quarter note, a quarter note, a set of eighth notes, and a quarter note) for their first measure only had the pitches of C, D, and G written down. Anthony's two sets of sixteenth notes for measure three were written by the group as "Ti-Ti, Ti-Ti, Ti-Ti, Ti-Ti" instead of "Tiki-Tiki, Tiki-Tiki" (i.e., four sets of eighth notes instead of two sets of sixteenth notes), and did not include pitches.

Throughout this compositional activity, one of four stages relative to the creative processes emerged: preparation, with the speculation of the incubation stage having emerged with regards to rhythmic coding. It was minimally evident that each group member's creative compositional processes did not progress in a linear fashion, but instead in an interchangeably cyclical manner, specifically between the preparation and illumination stages. However, the group had ruminated over their preparation stage before slightly progressing onward to their illumination stage, regardless of their objective to complete their A section melodies that day. I speculated this to be the case due to the insufficient timing that the students were given on this compositional activity, where the initial two-minutes of their compositional activity was dedicated to exploring different sounds/rhythms through improvisation, proceeding to the next two-minutes being used to create the first two measures of their melodies, and finally having their last five-minutes to create measures three and four of their melodies before class ended. In other words, the time constraints that were allotted to the students were only sufficient enough to allow them to explore through the different pitches and rhythms briefly; they were not nearly long enough to fully venture into the following stage of illumination, 
where the occurrence of revisions and edits to their composed melodies would happen. This was the case for Jane's group.

Specifically, within Jane's group, all four students had explored through different sounds and rhythmic patterns, but seemed to have gravitated towards Jane's initial rhythmic pattern of "Ta, Ta, Ti-Ti, Ta" (i.e., a quarter note, a quarter note, a set of eighth notes, and a quarter note) for their measure one even though she initially played it using just the keys of C and G. During this time of exploration, the group was in their preparation stage. Throughout most of the given time, it remained in this stage to create measures one through four of their melody. Nonetheless, I speculated that their creative processes progressed into their illumination stage regarding rhythmic coding since the group retained their initial rhythmic pattern of "Ta, Ta, Ti-Ti, Ta" for their first measure throughout the study.

Furthermore, while working on creating measures three and four, the group continued to experience their preparation stage. However, I again speculated that they had progressed into their illumination stage regarding rhythmic coding due to Aaron and Shawn's exploration with the two sets of sixteenth notes for measure three. My speculation was determined as a result of the group retaining both sets of sixteenth notes, one set on the A key and the other set on the $\mathrm{C}$ key as described by both boys in week two of the study, in their measure three on the final week of the study. However, their illumination stage during the second week of the study might have been prematurely forged as the group did not experience enough time within their preparation stage to fully explore through the various sounds and rhythmic patterns to determine their "aha" moment in their illumination stage, except for the rhythmic coding as mentioned above. 
Moreover, indications of enabling conditions and environmental influences were inherent throughout their preparation stage, particularly with the group members' differentiation of personalities. When it came to Aaron and Ashley, their personalities were prone to spontaneity and risk-taking while also applying their personal novelties and flexibilities to their exploration of pitches. Shawn and Jane can be considered individuals that have spontaneous/risk-taking attributes, but their craftsmanship and aesthetic sensitivity was noticeably apparent in their work ethics during the execution of this compositional task.

As per Springer's (2013) discussion on improvisation and composition in tandem to creative thinking, one of the prudent aspects of the Orff-Schulwerk is experiential learning through hands-on engagement which allows children to experience music interactively that promotes their creative thinking. Additionally, Hickey (2012) described the importance of providing sufficient opportunities for children to explore and manipulate various sound elements as it aids their creative thinking and sound-shaping, contributing factors to music-making activities such as composing and improvising. With regards to rhythmic coding, the instruments used in an Orff-based music classroom are prone to facilitate children's music-making activities as they recognize familiar sounds on the instruments while they reinforce their rhythmic notation or coding of the sounds they explored with, which was minimally evident in Jane's group as their explored rhythmic patterns during the second week of the study was retained in their final product of their composition. This rhythmic coding could have also been a result of their incubation stage, which my speculation determined it to be due to the group exploring through these rhythmic patterns without notating them in week two of the study, but later having these 
rhythmic patterns in their final creative product for the last week of the study. This time away allowed the group members' unconscious minds to incubate on the compositional activity by diverting their attention elsewhere, thus influencing their creative thinking positively towards their final creative product (Springer, 2013). Furthermore, Wiggins (2003) described that, although there is a representation of the interactions between and among these different phases, the social aspect within the outermost layer of her frame strongly influences the group's collective creative processes that result in their collective creative product.

Bassline, Melody, and Color Part. The last week of the study is here, and Ms. Smith asked the class to sit by a pair of instruments with their composition groups. She mentioned that the class would be skipping their regular warm-up drills today because "...we need to have that time to put everything [all the compositional elements] together." Ms. Smith began to describe how the objective of last class was to combine the bassline and melody together so that they could be played simultaneously for the final performance. She continued to say that today's objective was to review playing both the bassline and melody together so that groups could create their color part to add into their composition which would be the final procedure to the composition project.

Ms. Smith provided a musical imagery to the class by describing the color part as "the cherry on top of their ice cream," which she had mentioned throughout the last few weeks before its formal introduction the last week of the study. She continued to describe that the color part should not be played throughout the entire song, but instead it should be played where it will make the song "more beautiful and more exciting" like "a little triangle ding that happens every four measures." However, before the groups would 
begin on choosing their unpitched percussion instruments and creating their color part, they would be given ten-minutes to combine their basslines and melodies to be played simultaneously. Ms. Smith also elaborated that the groups should designate roles to those who will play each of the compositional elements so that those students could begin practicing their respective parts immediately. Their goal was for them to be able to successfully execute playing their assigned compositional elements simultaneously.

I walked over to Jane's group and asked if they would mind if I observed them today, to which they replied "that's fine." Jane and Shawn were the only ones in the group at that moment since Ashley was absent for the day and Aaron was not in the classroom until a few minutes later. Ms. Smith walked over to them since she thought they were to only two of the group here, so was going to help the group with the color part later on. For the moment, Ms. Smith had just asked Jane's group to share with her the split of designated roles. "I'm on the bass and she's the melody," said Shawn, to which Ms. Smith replied, "the melody's going to be [played] on the higher [pitched] instrument. So why don't you guys figure out how to adjust yourselves so that you're at the right instrument." Ms. Smith then announced to the class that "the bassline is always [played on] the larger instrument [and] the melody is always [played on] the smaller instruments" and followed that up by asking those that were playing to get on their respective instruments based on which part was designated for them to play.

Shawn had suggested to Jane that they speak through the bassline rhythm on pitches. When he began to speak the rhythm to what he thought was the bassline, he noticed that he was actually speaking the melody rhythm and pitches. He then quickly picked up the bassline sheet and Jane started to help Shawn with the bassline by speaking 
the pitches with him while he attempted to play the bassline, However, Jane had said the chord bordun part in reverse to what the group had formally notated, though the group had been playing it as she spoke it since the first week of the study (See Figure 4.4): “Okay, so it's: $\mathrm{C}|\mathrm{G}, \mathrm{C}| \mathrm{G}, \mathrm{G}-\mathrm{High} \mathrm{C}$, low $\mathrm{C}$. This is the notes that you have to play together. Play this together, twice. Play it together, $\mathrm{C}|\mathrm{G}, \mathrm{C}| \mathrm{G}$ twice. Together. Find the C...," affirmed Jane. "C's right here... It's C|G...," responded Shawn and Jane said "Find the G..." "Wait... it's... Oh my god," expressed Shawn as he attempted to play the bassline.

Jane noticed that Shawn was having a difficult time playing through the bassline and hitting other pitches that were not the $\mathrm{C}$ or $\mathrm{G}$ keys, so she suggested that he remove the B keys off of the xylophone to which I added "how about you take off the F's and the B's, Shawn?" While Shawn attempted to play through the bassline, Jane attempted to play through the melody where she began very well by staying in tempo and playing the pitches correctly, but slowed down the tempo during the two sets of sixteen notes in measure three. Ms. Smith then asked for a momentary "rest position" to allow each group to play their basslines to her to assure that all of the groups can successfully execute playing their basslines. At the same time that the groups were playing their basslines for Ms. Smith, Aaron arrived at the classroom where I informed him that I would be observing his group that day. When Ms. Smith called on their group, Shawn played their bassline very well by keeping the tempo of their combined bordun ostinato, but had played it slightly different pitch-wise than what was notated on their bassline sheet. This also occurred during the first week of the study where the group created their combined 
chord/broken bordun bassline and played it rhythmically accurate from what they formally notated, but slightly different pitch-wise (See Figure 4.4).

Shawn continued to practice the bassline on the xylophone while Jane was practicing the melody on the melody bell. Aaron was curious to know why Jane was playing the melody on the lower pitched keys of the melody bells rather than playing it on the higher pitched keys. "Because it starts to sound weird *played several keys on the higher pitched keys* see, it sounds weird and we don't like that," declared Jane to Aaron. Shawn then asked Aaron which composition part he would want to play, and added that they could both practice the bassline together because "it's very simple."

Jane's group continued to practice through the melody and the bassline before attempting to put both parts together to be played simultaneously. At this point, Aaron was practicing the bassline on his own, meanwhile Shawn was helping Jane with the melody, especially with the sets of sixteenth notes in measure three. Shawn vocalized "G, A..." "I still have no idea... Yeah. I don't know this," Jane had expressed with a look of frustration on her face. Shawn commented "well you mess up on the G." "Yeah, I know!" retorted Jane to Shawn.

The group continued practicing through both parts separately, but Jane mentioned to both Aaron and Shawn that they had to start learning how to combine the bassline and melody so that the group could play both parts simultaneously. As the group attempted to learn how to play both parts simultaneously, Jane continued to be the one responsible to play through the melody as Aaron and Shawn took turns playing through the bassline with Jane being on the melody part. However, the group's persistence on combining their bassline and melody together was halted when Ms. Smith asked the class to get into "rest 
position" once more so she could hear each group's bassline and melody played simultaneously. The time that Ms. Smith took to hearing each group's combined parts gave me the opportunity to converse with Jane about the melody, particularly about the sets of sixteenth notes in the third measure of their melody that she displayed having trouble playing through (See Figure 4.5). I asked Jane "is this a Ti-Ti or Tiki-Tiki," and she responded with "Ti-Ti." "How many beats do you have in that measure then," I ask as I point to measure three with my pencil. "Oh, I forgot," Jane said as she was blushing a bit from embarrassment, which I kindly inquired about the note value by asking "how many beats does Ti-Ti have?" "Two," Jane confidently answered and puzzled by her response I questioned her with “two, are you sure?" where she shook her head in disbelief saying "one." "If you have four Ti-Ti's that means you have four beats. Count with me..." as I pointed to measure three, “... one, two, three, four, five, six beats if these are Ti-Ti's," I explained, then added, "are you supposed to have six beats in a $\frac{4}{4}$ ?" She seemed a bit bothered and replied, "well she wrote this without describing it." "It should actually be Tiki-Tiki, Tiki-Tiki. So when you play this beat, it has to be faster...," I clarified, then added "do you know how to play a Tiki-Tiki?" In a confident manner, she picked up the mallets and replied with "I think I can play it faster," as she plays the sets of sixteen notes accurately, to which I grinly responded "mm-hmm."

\section{Figure 4.5.}

\section{Melody of Jane's Group}

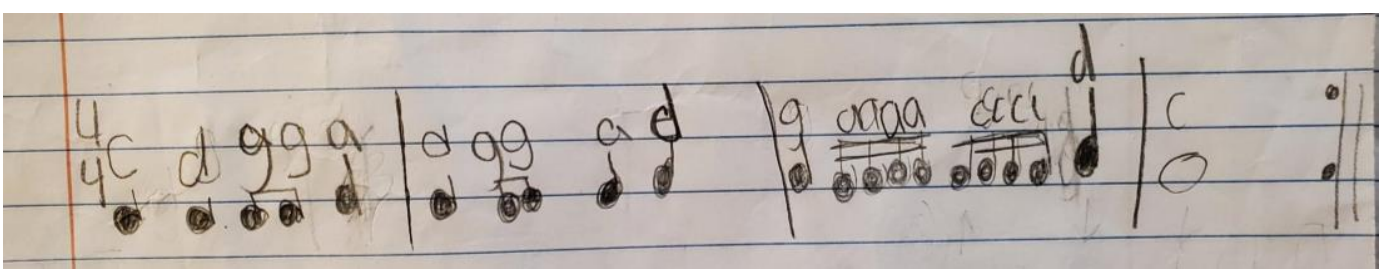


When Ms. Smith called upon Jane's group to play their bassline and melody together for her, Jane and Shawn seemed to be concentrated on their respective parts; Jane continuing with the melody and Shawn playing the bassline. Both Jane and Shawn began playing together in tempo, but Jane momentarily paused before the sets of sixteenth notes in measure three and also after the sets of sixteenth notes before continuing to play throughout the melody. "I think you need to look at the rhythm for that measure because you're adding a rest in there. You actually added two rests, one before the Tiki-Tiki and one after the Tiki-Tiki,” said Ms. Smith to Jane. Meanwhile, she thought Shawn had played extremely well, but in fact he had played a slightly different pitch-wise bassline although rhythmically accurate to what they notated, exactly like they had done since the first week of the study (See Figure 4.4).

Upon Ms. Smith hearing each group play through both their bassline and melody simultaneously, she presented several unpitched percussion instruments that were adequate "possibilities to add [as] a color part" to their compositional project. She also went through each of these unpitched percussion instruments demonstrating how each of them would sound and how they could be used as a color part before the groups had the chance to choose their preferred instrument for their color part:

You can add a small bell, which has a higher sound than the instruments that you're using. You can add just a nice little clink. You can add an actual pattern which is more complicated, but it's really up to you... I have two bells and I have two different kinds of mallets. I have a softer mallet, which is made of rubber so that it doesn't overpower anything. If you're going to do a pattern, I suggest using the rubber mallet, not the hard one. The hard one is more for just a little clink somewhere. We also have the triangle. I couldn't find my other triangles, so if someone wants to use it there's only one group that can... We have tambourines, as well. I have two of them here. This one, I think you have to be really careful about using it... But if you use it correctly, it could be cool. There are claves in here. Please remember that when you're using the clave, you're going to make a 
little cave. You're going to place the clave on top of the cave, not inside the cave. Okay? There's two sets in there. I have some shakers in here, as well. Again, I would not use the shaker the whole entire time. I would choose wisely where to put it in. I also have the guiro. If you're going to use it, you're going to take the pointer and thumb, put it in the hole. You're going to use this as the scraper.

However, Ms. Smith had a limited number of unpitched percussion instruments to lend to each group. This not only meant that many groups would have to settle for an unpitched percussion instrument that was not their preferred instrument, but only a few groups would get to actually use the preferred instrument that they desired to use. Ms. Smith also had to provide specific parameters to certain instruments being used so that when the groups would begin to incorporate these instruments as their color parts it would not overpower the overall sound of their multi-layered compositional project, to which the rhythmic direction that each group would choose also contributed towards the parameters they had to abide by for their color part depending on the instrument they had.

Ms. Smith began to ask one group at a time which instrument they were planning to use for their color part. While Jane's group waited to be called on, I was curious to know which instruments they were thinking or planning on using for their color part. Shawn had mentioned that he would like to use the clave for their color part, which Jane and Aaron seemed to be comfortable with Shawn's decision. "I personally like the triangle because it kind of rings after you play it," I said to which Shawn expressed, "I know, but there's only one [triangle] and I don't want to get into a fight for it. There are already a few arguing for it." I nodded in agreement to his statement, but Aaron had to blurt out "I could get it" and Jane proclaimed that "yeah, Aaron will fight for it," which made us all laugh. 
When it was the group's turn to pick their color part instrument, Ms. Smith mentioned that all the claves were gone and all she had left were the guiro, the tambourine, the slapstick, and rhythm sticks. Jane chose to go with the rhythm sticks, but Aaron immediately seemed frustrated with her decision and said that the rattle drum would have made them "be so loud" instead of the rhythm sticks. Since I was not sure what Aaron meant by that statement, Shawn explained to me what he was talking about which sparked a conversation between me and the group regarding certain unpitched percussion instruments at Animal Kingdom. However, the conversation quickly drifted towards talking about the particular rides at Animal Kingdom and how Aaron spent "like four hours" in line for the Pandora boat ride and the other Pandora ride about eight hours in line. He also mentioned that he did not like Animal Kingdom all that much and Shawn expressed how he had never been to Animal Kingdom before "but I've been to all the other ones."

While Jane continued to experience difficulty playing through the melody, especially the third measure, Aaron asked her if he should play the rhythm sticks using the same rhythmic pattern as the bassline (i.e., quarter note, quarter note, a set of eighth notes, quarter note). However, Shawn emphasized that this would be the color part which I elaborated on by stating that "it's the cherry on top" of their ice cream like Ms. Smith had reiterated several times throughout the course of the study. "I don't like cherrie though," replied Aaron. I then engaged in a conversation with Aaron to try to explain how the color part works with the rest of the compositional elements to create this overall multi-layered composition. "Do you like steak?” I straightforwardly asked Aaron. Almost immediately and proudly he answered, "hell yeah I like steak!" "Okay..." I say with a 
grin, “do you put any sauce on your steak?" I inquired to continue the conversation, which Aaron quickly responded with "sometimes ranch," while seeming to almost salivate from the thought of having steak. I giggled a little bit since I, too, seemed to have almost salivated from thinking about steaks. "Okay, so think of this as, this is your steak..." I explained while pointing to the group's melody sheet, then energetically added "and then you are the little bit of ranch to your steak." Hoping he understood my analogy, I asked, "Do you get what I'm saying?" Aaron gave me an affirmative nod that he understood my analogy, and understood his role in the overall compositional project.

My steak metaphor kindled Jane's wittiness to come out which sparked a miniature joking-interaction between her and Aaron. She proudly exclaimed to be "the steak" and Aaron jokingly responded with “where's the fork?" Jane's witty retort was to express that Aaron was the fork, though he humorously claimed that Jane would eat the steak with her bare hands. After this amusing interaction and some shared laughs, Aaron affirmed that he was aware and understood his role of the color part within the compositional project much clearer now.

Ms. Smith asked the class to return to "rest position" once more, and announced that she would like to hear each group's color part to provide feedback before the final performance that was quickly approaching. Since the group did not have enough time to plan out what Aaron would play for their color part, Shawn suggested that Aaron play about a quarter percent of the time. "That's smart, do it at the end," said Aaron as Shawn demonstrated what he was thinking of Aaron doing for the color part. However, when it was their turn to play all three compositional elements together for Ms. Smith, Aaron did 
not play or even attempted to play what Shawn had suggested. In addition, Jane was still struggling to play through the sets of sixteenth notes in the melody's third measure. Ms. Smith asked Aaron where he was considering placing the rhythm sticks for their color part, but he replied with "we still haven't figured it out yet." Ms. Smith then suggested that their color part be based on their melody and added that she "... would see what part of the melody you think needs to be special, and that's where you end up playing [the rhythm sticks].”

"In five minutes, we're doing our final performances, so you have five minutes to fix any issues," announced Ms. Smith. Jane immediately started suggesting where Aaron could place the rhythm sticks, but Aaron wanted to switch designated roles to play a different compositional element because the color part was confusing to him. Shawn expressed that he would prefer to continue playing the bassline, so Jane was comfortable swapping positions with Aaron since she was already working on creating the color part. As she continued to work through the color part, Jane mentioned that she had "... a feeling it's good to do it [the rhythm sticks] with these two [measures one and two] and the last part [measure four]." I was curious to know why she thought the rhythm sticks would fit well in measures one, two, and four for their color part. "Because it's like C, D, G-G, A. D, G-G, A, D. G, *sings* A-A-A-A, C-C-C-C, D. C, C, C, C, ” replied Jane while she demonstrated her exploration of the rhythm sticks being played as a quarter note throughout measure one, two, and four. Although her explanation was very detailed, I was eager to know why she sang four C's in measure four when their formal notation has a whole note for the fourth measure (See Figure 4.5). Confidently, Jane responded, 
"this C is like..." while she played four quarter notes on the C key. "Okay, but that's not what you have written down in your melody," I declared, which Aaron argued by stating "you can't do that though." "But guys, there's a whole note at the end. We should just do that four times," suggested Shawn. Jane replied, "but I don't know how to put it loud," admitting to not knowing how to produce a louder sound for the whole note. "You're playing four quarter notes, and what do you have written here?" I asked Jane. She questionly responded, "one... whole note." "Then put four quarter notes then!" argued Aaron while I said, "whole note." I continued speaking to Jane, "and how many beats does a whole note have?" "Aaron blurted "one." "One" I ask while having a puzzling look on my face. "Four," Aaron firmly said. "Exactly..." I said, then added for clarification, "so you hold it for four." Aaron then suggested, "she hold it, then you play four."

As Jane continued to practice the color part, she was helping Aaron play through the melody while Shawn practiced the bassline. Like Jane, Aaron was having a difficult time playing through the sets of sixteenth notes in measure three of their melody. Jane kept repeating "G, A-A-A-A, C-C-C-C" while maintaining a steady quarter note beat with the rhythm sticks so that Aaron could play through the sixteenth note pairs with rhythmic accuracy. While Aaron and Jane played through the melody and color part together, Jane persisted on with her original exploration of the rhythm sticks being played on a steady quarter note beat in measures one, two, and four. Nevertheless, Shawn reminded Jane to not “... hit it [rhythm sticks] so much," where she replied, “...just the first two [measures] and last one." Before Ms. Smith asked the class to get into "rest 
positions" for their final performances, Jane's group worked together to combine and play through each of their respective compositional elements simultaneously with their group member's counterparts.

Ms. Smith explained a few rules to her assessing the final performances and began by stating that each group was "allowed one restart to play it again" if the group chooses to do so. She also stated that she would be counting each group in unless otherwise that group would like to count themselves in. Since Annie's group was the only one to mention that they would count themselves in, Ms. Smith began the final performance evaluations with their group. As Ms. Smith heard and assessed each group's performance, Aaron continued to practice the melody by speaking the rhythm on pitches. Aaron also wanted to make sure of what his group had decided for the last measure of their melody so that he could play it accurately, which Jane told him to play the whole note while she played four quarter notes with the rhythm sticks in measure four.

When it was their turn to play for their final performance, Aaron was on the melody, Jane was on the color part, and Shawn was on the bassline. Aaron, Shawn, and Jane played extremely well together and maintained a steady tempo, with the exception of Aaron slightly misplaying the first set of sixteenth notes in measure three but quickly recovered to stay in tempo with his group members' respective parts. Ms. Smith was very pleased with their performance of the compositional project and even mentioned that their composition had improved with how Jane situated the color part into the compositional project. As Ms. Smith went on to congratulate the class for their extraordinary performances, I was congratulating the group for their magnificent 
performance and teamwork.

Three of four stages relative to the creative processes emerged, which were preparation, illumination, and verification, with the speculation of the incubation stage having emerged with regards to musical imagery. It was evident that each of the group member's creative compositional processes did not progress in a linear fashion, but instead in an interchangeably cyclical manner, specifically between the stages of preparation, illumination, and verification. However, to effectively illustrate each of the creative processes within my findings, I will begin by describing the stages that emerged when Jane's group was practicing to combine the bassline and melody to be played together, and then I will describe the stages that emerged when the color part was added into their compositional project.

With regards to the bassline and melody, the apparent stage that emerged was the verification stage. Within this stage, the group was continuously rehearsing and polishing their bassline and melody as they play both parts simultaneously. However, when it came to the bassline, Shawn played a slightly different pitch-wise bassline that was notated on their bassline sheet, although rhythmically speaking the notation was accurate. This was also the case during the first week of the study when the group played their combined bordun bassline. It was evident that, when having to sight-read the pitches of the bassline, Shawn played a reversed version of the pitches notate, thus displaying a lack of reading ability in formal notation with regards to pitch accuracy; an indication of enabling skills inherent within this specific stage. Notwithstanding, in terms of rhythm accuracy, their formal notation was accurate (See Figure 4.4) which possibly meant that the group had 
potentially learned to play their bassline via rote more than via note while progressing through the stages of preparation, illumination, and verification during the first week of the study. When it came to the melody, Jane and Aaron both had difficulty playing through the third measure, particularly the sets of sixteenth notes (See Figure 4.5), also an indication of enabling skills inherent within the verification stage. Since both Jane and Aaron were playing the sets of sixteenth notes as if they were eighth note pairs, they possessed a lack of conceptual understanding of their rhythmic notation as they experienced "reading" the complex rhythm incorrectly. In addition, they possessed a lack of musical aptitude within the context of playing through complex rhythmic patterns, which is also an indication of enabling skills inherent within this specific stage.

With regards to the color part, three of four stages relative to the creative processes emerged, which were preparation, illumination, and verification, and the speculation of the incubation stage having emerged with regards to musical imagery. These creative compositional processes progressed in an interchangeably cyclical manner rather than a linear fashion, specifically between the stages of preparation, illumination, and verification. However, given the limited number of unpitched percussion instrumentation that Ms. Smith had in her possession, Jane's group settled for the rhythm sticks, which was not their first choice. This not only created an environmental limitation inherently known as an enabling conditions within Webster's (1990/2002) model of creative thinking in music, but also tampered with the group's preparation stage since they had to shift their plans in another direction as their preferred unpitched percussion instrument was not available as well as their intrinsic motivation. While in their 
preparation stage, Aaron's exploration with the rhythm sticks using the same rhythmic pattern as their bassline (i.e., Ta, Ta, Ti-Ti, Ta or quarter note, quarter note, a set of eighth notes, quarter note) would not have been adequate enough for their color part. By using a metaphorical analogy, much like Ms. Smith's "cherry on top of the sundae" analogy, I helped convey to Aaron the function of the color part and how it relates to the overall compositional project; the steak being the melody and the ranch sauce (Aaron mentioned he liked ranch) is the color part. This steak metaphorical analogy served as a musical imagery within the context of the group's incubation stage, specifically Aaron's incubation stage, as their stages of preparation, illumination, and verification interacted between and among themselves. Their progression into their illumination stage occurred when Jane's "feeling" of playing the rhythm stick on a steady quarter note beat throughout measures one, two, and four was accepted by the group, which naturally progressed into their verification stage with the other respective compositional elements.

To collectively summarize the above findings: Three of four stages relative to the creative processes emerged, which were preparation, illumination, and verification, with the speculation of the incubation stage having emerged with regards to musical imagery. Evidence of the group's creative compositional processes suggest a progression through an interchangeably cyclical manner rather than a linear fashion, specifically between preparation, illumination, and verification. When having to sight-read the pitches of the bassline, Shawn played a reversed version of the pitches notated, displaying a lack of reading ability in formal notation with regards to pitch accuracy. However, the formal notation was rhythmically accurate (See Figure 4.4), thus concluding that the group might 
have potentially learned to play their bassline via rote more than via note as they progressed through the stages of preparation, illumination, and verification. These were all inherent indicators of enabling skills within the verification stage. When sight-reading their melody, both Jane and Aaron played the sets of sixteenth notes like eighth note pairs, displaying a lack of conceptual understanding of the rhythmic notation while "reading" the complex rhythm incorrectly, as well as displaying a lack of musical aptitude within the context of playing through complex rhythmic patterns. These were also inherent indications of enabling skills inherent within the verification stage. The group struggled to propel into the other stages when creating the color part besides their preparation stage, but my metaphorical analogy served as a musical imagery within their incubation stage to progress between and among the preparation, illumination, and verification stages. Hargreaves, MacDonald, and Miell (2012) claimed that the concept of imagination focuses on the "internal mental processes" which facilitate one's mind to produce a creative outcome, classifying one's imagination as a more inclusive term (as cited in Wang and Springer, 2013). In Webster's (1990/2002) model of creative thinking in music, he states that enabling skills and enabling conditions are factors influencing the creative processes that move from divergent-to-convergent thinking. Nonetheless, my speculation was not that their creative processes were entirely influenced by a lack of musical aptitude or conceptual understanding as outlined in Webster's (1990/2002) model of creative thinking in music. Furthermore, my belief is highly predicated upon a contributing fact that the group explored, revised, and rehearsed on an Orff-based instrument (Springer, 2013), which most likely facilitated their music-making by allowing them to easily recognize familiar sounds while simultaneously reinforcing their 
rhythmic coding (Frazee \& Kreuter, 1987), but it was most likely lost in translation when having to formally notate the pitches for their bassline or sight-read the rhythmic notation of their melody's third measure.

\section{The Teacher and Her Perspective}

Ms. Smith. Ms. Smith is an energetic and enthusiastic elementary and middle school music teacher who possesses all three level certifications in the Orff-Schulwerk approach. Her daily musical instruction fluoresces with boundless Orff-based activities that incorporate imitation, exploration, improvisation and composition with music, movement, speech, and/or drama while also including various pitched and unpitched Orff instrumentations into her curriculum. Although Ms. Smith has a classical music background consisting of choral ensemble, studio-based music learning, and choral statewide competition, she has altered her teaching ethics to be able to "evolve with the kids [the students]" which requires becoming familiar with unknown territories that kids are involved in nowadays. Since she first began teaching general music, Ms. Smith has developed a well-diverse Orff program into her music curriculum befitting all of her students, and has also established several afterschool ensembles such as her modern rock band and Orff ensemble.

Warm-ups. Ms. Smith usually begins her music classes like any other day, going through her daily warm-up drills with her students. She typically begins to sing their Welcome Song (i.e., Sol-ri-da) which incorporates simultaneous singing and hand movements. Since their Welcome Song has an A and a B section, she first goes through the A section then introduces the B section of the Welcome Song before demonstrating 
both parts being sung adjacent to one another. Ms. Smith then transitions into another warm-up I titled "rhythmical call and response." This exercise features clapping and patting in the duple/triple meter while she "calls" different rhythm syllables and her students imitate her as their "responses." When Ms. Smith goes through any of her "theory section" exercises, she begins with what she refers to as "Name That Note/Rest." In this particular exercise, Ms. Smith asks certain questions about different note values to which the students would raise their hands to answer. She usually follows that up by writing a rhythmic example she calls "Rhythm of the Day." Ms. Smith typically goes through the rhythmic example with her students as a way to help them familiarize themselves with the introduction of certain synchronized beats they have not spoken through yet, such as "Ti-Tiki." Once her students have been formally introduced to the new synchronized beat, Ms. Smith adds different dynamics and accent marks to the example, then has her students speak through the example once more articulating the dynamic markings added. What follows after the rhythmic example is another "call" and "response" with her students, but with her singing different melodic rhythms using solfege and Curwen hand signs that represented the pitches sung. She usually concludes her warm-up/exercise drills with her students singing a $\mathrm{C}$ major scale using solfege and Curwen hand signs. However, she started to divide her class into two sections, having one group singing the $\mathrm{C}$ major scale using solfege and Curwen hand signs and when that group gets to the third degree of the scale (i.e., Mi) the other group starts singing the scale to create a harmonization between both sections. Once the second group arrives at the end of the scale, both sections switch parts so that the other group gets to harmonize with their counter-group when they hear the third degree of the $\mathrm{C}$ major scale being sung. Her 
strategy with the warm-ups can be considered as "exposing them [the students] to certain things... [like the] interval stuff using solfege" which is the students' first experience with melody. Ms. Smith further elaborates that these "little building blocks" are later used to expand on those higher order processes, like composition and improvisation. This is evident throughout her warm-ups, such as when she explains the $\mathrm{C}$ pentatonic scale that the students are using to create their melody or the harmonization between the split class to distinguish the several sounds being sung at once. These are all what she refers to as "very Orff-y" based strategies which are instantly gratifying to children as they respond quickly to it. Ms. Smith also mentions that "kids learn through play" before they are ever formally introduced to any formal notation or sight-reading, which is why she is very fond of beginning to teach through rote first before "putting visual notation to music."

Teaching Process. As the study took its course, Ms. Smith continuously elaborated on what each group would be working on each week. She would begin by stating the objective of the day for the compositional activity being worked on, such as creating the entire bassline or combining both bassline and melody to be played together. Ms. Smith also had several parameters to include in each week's compositional activity. Some examples of these parameters included having certain rhythmic patterns used in one of the four measures in the melody (i.e., sets of sixteenth notes and triplets could only be added to measure three, and simple rhythms for measures one, two, and four), different borduns would use certain key bars (e.g., broken borduns could only use "big C" and "big G"), and the color part could not be played throughout the entire song because it should not take away from the entire composition. In addition, Ms. Smith 
provided a few metaphorical analogies towards the melody and color part, which served as vivid pictures that the students could think back on when working through those compositional activities.

As previously mentioned, the building blocks that Ms. Smith has provided her class with regards to experiential rhythmic/melodic learning are designed to assist the students with higher order processes, like composition. Specifically, their first composition project was a one-line rhythmic only composition, in which the students had to write a four measure rhythm and choose the instrumentation (e.g., pitched or unpitched) that they would perform their composition on. Ms. Smith strongly believed that this “...experience beforehand was really beneficial. I think that they would've struggled a lot more with this [multi-layered] project had they not composed rhythmically prior to doing it." It was evident that many groups were exceptional rhythmic readers, as she stated in our interview. However, Ms. Smith proclaims that due to her students having to"...combine the rhythm and the pitches... that's really hard for them... they're amazing at reading rhythm but then if I add pitches to it, that's a big leap" for them to be able to fully sight-read rhythmically and melodically, which was a reoccurring characteristic among the students. Many students did seem to possess a lack of musical aptitude and conceptual understanding with regards to sight-reading and reading ability to formal rhythmic/melodic notation, in spite of Ms. Smith claimed that her students are good "rhythm readers." In addition, Ms. Smith mentioned that the rhythmic direction that the group's had decided to go with dictated "what they were going to do melodically" since many of the students have difficulty connecting rhythm and pitches together as mentioned above. Moreover, she stated that this project did test her students' reading 
abilities, “....which some of them thrived at and for others it just wasn't their thing, which is fine" and added that "there were a lot of different elements to the project and not everyone's going to be great at every single element."

The course of the study involved several compositional activities that were individually undertaken before combining each element into an extensive compositional performance. Each of these compositional activities had their own complex attributes that presented a challenge towards each of the groups regardless of its difficulty. Although Ms. Smith stated that "the melody was more challenging," she also mentioned that the bassline and color part were the least frustrating aspects of this composition project for her students. She further elaborated that these building blocks she provides encourages exploration, specifically on the instruments, that translates into students anticipating their finished creative product, which is like an "A-ha' moment" for them as well as an exciting and instantly gratifying sensation to them. However, since the melody required students to think of their overall musical shape, including different rhythmic/melodic attributes such as organizing pitch sequences within rhythmic constraints (e.g., complex rhythms in their measure three; sets of sixteenth notes and triplets), to transition their thoughts into formal notation while also being vigilant to the other elements when playing simultaneously with the melody constitutes as "a very higher order thinking." This higher order thinking in composition is described by Ms. Smith as "a good issue because they're now practicing having to work through something that's more difficult... [where] all those little struggles in between" insinuates their overall learning experience.

Her teaching process induces her students to navigate through their creative thinking stages as they engage in their music-making process, which also promotes 
creative novelty, originality, and the development of one's musical identity (Hickey, 2012). According to Webster's (1990/2002) model of creative thinking in music, the interrelationship between product intention, thinking process, and creative product function together to influence creative musical thinking. The divergent-to-convergent thinking, specifically the stages moving between preparation, incubation, illumination, and verification, is the bridge that gaps the transition between product intention and creative product. In addition, Webster states that enabling skills and enabling conditions are factors that inherently influence the creative processes moving from divergent-toconvergent thinking, as found in Annie's and Jane's groups. Furthermore, Wiggins (2003) model named the frame described various characteristics of composing, such as organizing, evaluating, revising, refining, and rehearsing musical ideas, that interact between and among themselves, which also aligns with the interchangeable cycle between the preparation, illumination, and verification stages found in Annie's and Jane's groups. Moreover, the metaphorical analogies used by Ms. Smith served as musical imageries with the context of the groups' incubation stage as the interconnection between the preparation, illumination, and verification stages progressed towards their creative product.

Orff-Schulwerk. Ms. Smith typically has a boundless amount of Orff-based activities that incorporate imitation, exploration, improvisation and composition with music, movement, speech, and/or drama while also including various pitched and unpitched Orff instrumentations into her music instruction. Plenty of Ms. Smith's warmups/exercises are enriched with the approach's implementation by "teaching through rote first before putting visual notation to music" and "giving them these... musical 
vocabulary" which she describes as instantly gratifying to her students. "Kids respond to it [Orff-based instruction] very quickly," claims Ms. Smith who also elaborated on children "learn[ing] through play" because it comes naturally to them as they learn by experiencing different factors within her Orff-based curriculum.

Although Orff-based instruction is highly predicated on exploration and experiential learning, this multimodal, hands-on process builds on the fundamental learning of music concepts while simultaneously enhancing children's creative musicianship (Shehan, 1986). "It’s layering," said Ms. Smith, elaborating on how Orffbased learning builds off of "exposing them [the students] to certain things," such as her melodic interval warm-up which was her students' first melodic experience through rote learning. Those "little building blocks" that she provides are expanded or layered on top of one another to reach a higher order of thinking, as required for the music-making process. Frazee and Kreuter (1987) stated that "Orff instruments provide an invaluable means of exploring all of the elements of music" which facilitates children's rhythmic and/or melodic composition.

With regards to the composition project, Ms. Smith stated that the exploration phase of the project was the shortest phase that the students experienced because they have had several opportunities to explore while working on other projects. However, she stated that the writing and revising stage "ended up being the longest portion of their project." Ms. Smith also mentioned that some of her students struggled with rehearsing through their creative products because "notation might have been an issue for some of them." In terms of the approach's implementation, this is counterintuitive to the study conducted by Frazee and Kreuter (1987) who stated that Orff instruments could facilitate 
children's melodic composition through sound recognition while reinforcing rhythmic notation. Springer (2013) also elaborated that children's divergent and convergent thinking are enhanced through the deliberate and sufficient exploration of Orff instrumentation, aiding their creative thinking development. Additionally, Webster (1990/2002) stated that several enabling skills and enabling conditions could influence the creative processes that move from divergent-to-convergent thinking. Annie's group was one of the few groups that I speculated to have had a negative effect on the outcome of their creative product due to the nature of their environmental influence consisting of conflicts among the girls, thus resulting in a potential impact of their creative novelty, craftsmanship, and aesthetic sensitivity. This did not seem to be the case for other groups and some of them even enjoyed it, which was mentioned several times throughout the focus group interview. Ms. Smith elaborated that:

... Working in groups is a big deal and I think that's hard for a lot of them... Orff is very group-based... I think the social interactions, that was probably a challenge for some of them, whether it was because they are used to taking control and they want to do everything or the opposite; they're shy and they don't know how to speak up and contribute to the process.

Nevertheless, these contributing factors within the mentioned studies cannot be objectively determined due to the subjective nature that this study presents. Moreover, Hickey (2003) discussed the importance to provide an encouraging and nurturing environment within the music classroom to cultivate creative musical-thinking while considering the act of music-making a "depth over breadth and quality over quantity" factor no matter the compositional activity, which was noticeable within Ms. Smith's musical instruction that involved experiential learning prominent within an Orff-based music classroom. 


\section{CHAPTER V: \\ DISCUSSION AND IMPLICATIONS}

This chapter will present the stages that emerged across both groups within the Orff-based elementary music class and link those stages to the broader research literature. I will begin by restating the study's purpose and research questions, after which I will provide a descriptive analysis for each of the guiding questions with interpretations of these findings. The chapter will conclude with implications for research on children's musical creativity in music composition, music teaching and learning, and the compositional process through the Orff-Schulwerk approach.

\section{Restatement of Purpose and Research Questions}

The purpose of this study was to explore the creative processes of elementary school students in music composition through the Orff-Schulwerk approach. Specifically, this study will examine their creative processes through the framework of divergent to convergent thinking within Webster's (1990) model of creative thinking in music. The following central questions were used to guide this study:

1. What is the nature of children's creative processes during an Orff-based music composition task?

2. What are the children's perceptions of their creative processes employed during an Orff-based composition task?

3. What are the teacher's perceptions of teaching and learning music composition through the Orff-Schulwerk approach? 


\section{Descriptive Analysis and Discussion}

\section{Research Question One: What is the nature of children's creative processes}

during an Orff-based music composition task? Three of the four stages relative to the creative processes emerged: preparation, illumination, and verification. In addition, the incubation stage was speculated to have emerged in relation to musical imagery and rhythmic coding. Evidence shows that the creative compositional processes of the participants did not progress linearly, but instead in an interchangeably cyclical manner.

Bassline. With regard to the bassline compositional activity, the preparation stage was the first apparent creative process among both Annie's group and Jane's group. Both groups began their bassline compositional activity by planning out which borduns they were going to explore and started exploring with different rhythmic patterns for their ostinatos. This stage was very fast-paced and was the least time consuming throughout both groups, possibly stagnating their overall potential to produce an evermore creative bassline for their compositional projects. However, the illumination and verification stages of both groups overlapped by interacting between and among each other, which Wiggins (2003) elaborated on in her model called the frame. Both the illumination and verification stages were the two stages that the groups spent the most time on while they revised, edited, and formed new ideas for their basslines. The groups also subsequently rehearsed and polished their ostinatos for a successful execution at the end of class. Therefore, the evidence of the findings determined that the creative processes of both groups traveled on a spectrum between the stages of preparation, illumination, and verification during the bassline compositional activity. This coincides with Landis and Carter's (1972) study where they elaborated on the subsequential act of improvising on 
Orff-based instruments, which encourages students to use compositional devices such as borduns, ostinatos, and rondos that are distinctly elemental within the Orff-Schulwerk approach.

During this compositional activity, Jane's group had played a bassline that was slightly different pitch-wise to what they had notated on their bassline sheet, although rhythmically speaking the notation was accurate to what the group had played for Ms. Smith towards the end of class. Meanwhile, Annie's group traveled on a spectrum between their illumination and verification stages for this compositional activity since they had to edit their bassline due to writing it in $\frac{3}{4}$ instead of $\frac{4}{4}$, which Ms. Smith assisted by advising them to add a rest at the end of each measure in their ostinato. This group then rehearsed and polished their bassline before combining and playing it with the other compositional activities. It was apparent that, when Jane's group had to sight-read the pitches of their bassline, they played a reversed version of the pitches notated, thus displaying a lack of reading ability in formal notation with regards to pitch accuracy. When it came to rhythm accuracy, their formal notation was accurate, which could have potentially resulted from learning to play their bassline through the approach's implementation of elemental rote learning more so than note learning while going through the stages of preparation, illumination, and verification. In Webster's (1990/2002) model of creative thinking in music, he states that enabling skills and enabling conditions could also influence the creative process that moves from divergentto-convergent thinking. For Annie's group, enabling skills such as conceptual understanding regarding rhythmic and formal notation were inherent in playing the bassline with the melody simultaneously. Although Frazee and Kreuter (1987) states that 
Orff-based instruments can facilitate music-making activities through sound recognition while reinforcing rhythmic coding, such was not the case with Annie's group since their conceptual understanding of rhythmic and formal notation fell short when it came to sight-reading the bassline with the melody. In regards to Jane's group, my speculation was not that the group's creative process during this compositional activity was entirely influenced by a lack of musical aptitude or conceptual understanding as outlined in Webster's (1990/2002) model of creative thinking in music. Moreover, my belief is highly predicated upon a contributing fact that Jane's group explored, revised, and rehearsed specifically on an Orff-based instrument (Springer, 2013) and used compositional devices such as different borduns and ostinatos on this Orff instrument (Landis \& Carder, 1972), which is a fundamental aspect within the Orff-Schulwerk approach. This may have likely facilitated their music-making by allowing them to easily recognize familiar sounds while simultaneously reinforcing their rhythmic coding towards their exploration (Frazee \& Kreuter, 1987), but it was most likely lost in translation when having to notate the pitches they were using for their bassline. Counter to Jane's group, Annie's group also had indications of enabling conditions and environmental influences inherent within the specific stages of illumination and verification due to arising conflicts between Nicole and her peers when she wanted to change the bassline despite the teacher helping them fix it to delineate a $\frac{4}{4}$ time signature. This conflict may not have had a great influence on Annie's group at that moment. Contrarily, other conflicts did affect their group's overall motivation within their environment, which had impacted their overall creative novelty, craftsmanship, and aesthetic sensitivity. 
Melody. Throughout the melody compositional activity with respect to Annie's group, three of the four stages relative to the creative processes emerged: preparation, illumination, and verification. However, the group experienced going through their "preparation" and "illumination" stages as "artificial creative processes" due to Ms. Smith's scaffolding the parameters towards their "exploration" and "refining" of their melody. These stages were very fast-paced given the nature of the teacher's scaffolding that facilitated their "exploration" and "refining" of their melody. In addition, their "preparation" stage constituted as an "illumination" stage as Annie's group "created" their melody while Ms. Smith walked them through the rhythmic patterns and pitches appropriate for them to use in each measure. When combining the bassline and melody, Annie's group traveled on a spectrum between their illumination and verification stages; the group had to revise their bassline by adding rests at the end of each measure, and then had to rehearse/polish each part before combining and playing both parts simultaneously. Furthermore, indications of enabling conditions and environmental influences were inherent within these specific stages as Annie's group experienced several conflicts among themselves, particularly among Nicole and her peers. These conflicts were frequently noticeable among Annie's group, thus affecting their overall motivation within their environment. This also impacted their enabling skills particularly their creative novelty, craftsmanship, and aesthetic sensitivity. Moreover, enabling skills such as conceptual understanding regarding rhythmic and formal notation were inherent when playing the bassline and melody simultaneously. In Webster's (1990/2002) model of creative thinking in music, he states that enabling skills and enabling conditions are factors influencing the creative process that moves from divergent-to-convergent 
thinking. These indicators of enabling conditions and enabling skills may have potentially stagnated each of the group member's production towards creatively composing their melody, which possibly resulted in a lack of enjoyment among the group members. In addition, Frazee and Kreuter (1987) states that Orff-based instruments can facilitate music-making activities through the elemental factor of sound recognition while reinforcing rhythmic coding within the Orff-Schulwerk approach. However, this was not always the case with Annie's group since their conceptual understanding of rhythmic and formal notation fell short when having to sight-read the bassline and melody, more specifically with sight-reading the melodic notation of their third measure that included more complex rhythmic patterns.

Throughout the melody compositional activity with respect to Jane's group, three of the four stages relative to the creative processes emerged: preparation, illumination, and verification, with speculation that the incubation stage had emerged in relation to rhythmic coding. It was evident that each of the group member's creative compositional processes did not progress in a linear fashion, but instead in an interchangeably cyclical manner, specifically between the stages of preparation, illumination, and verification. However, Jane's group spent a majority of their time ruminating over the preparation stage before progressing onward to the other stages. This may have resulted in time constraints allotted at the beginning of creating their melody, which seemed only sufficient enough for Jane's group to explore through the different pitches and rhythms briefly, therefore not progressing fully into the illumination stage until the following week of the study. Hickey (2012) describes the importance of providing sufficient opportunities for children to explore and manipulate various sound elements as it aids 
their creative thinking and sound-shaping, which are contributing factors to musicmaking activities such as composing and improvising. In tandem, Springer (2013) discusses how improvisation and composition are the most conspicuous products of creative thinking, prudent of the Orff-Schulwerk approach which is based on the fundamental aspect of experiential learning through hands-on engagement that promotes creative thinking. Additionally, indications of enabling conditions and environmental influences were inherent throughout their preparation stage, particularly with the differentiation of personalities among the group. These enabling conditions and environmental influences could have positively contributed to the group's progression towards their illumination stage, which is an integral component of divergent-toconvergent thinking as described in Webster's (1990/2002) model of creative thinking in music. With regards to rhythmic coding, I speculated that Jane's group slightly progressed into the illumination stage from the preparation stage since the group retained certain rhythmic patterns (i.e., "Ta, Ta, Ti-Ti, Ta," and both sets of sixteenth notes on the A and $\mathrm{C}$ key) from when they first began creating their melody. This rhythmic coding may have been a result of their incubation stage, which I also speculated and determined through their exploration of these rhythmic patterns without its notation but retaining them to later notate them on paper by evidence of their final performance. Since the instruments used in an Orff-based music classroom are prone to facilitate music-making activities through fundamental hands-on learning, children naturally gravitate towards recognizing familiar sounds on the instruments while reinforcing their rhythmic notation of the sounds that are being explored. Furthermore, taking time away from a task allows one's unconscious mind to incubate on that task by diverting their attention elsewhere 
which influences their creative thinking positively towards their final creative product (Springer, 2013). Still, Jane's group had difficulty playing through the third measure of their melody, which indicated that enabling skills were inherent within their verification stage. When sight-reading their melody, Jane's group displayed a lack of conceptual understanding of the rhythmic notation while "reading" the complex rhythms incorrectly, as well as displaying a lack of musical aptitude within the context of playing through complex rhythmic patterns. In Webster's (1990/2002) model of creative thinking in music, enabling skills and enabling conditions are factors that could influence the creative processes which move from divergent-to-convergent thinking. Nonetheless, my speculation was not that their creative processes were entirely influenced by a lack of musical aptitude or conceptual understanding as outlined in Webster's (1990/2002) model of creative thinking in music. Furthermore, my belief is highly predicated upon a contributing fact that the group explored, revised, and rehearsed on an Orff-based instrument (Springer, 2013), which most likely facilitated their music-making by allowing them to easily recognize familiar sounds while simultaneously reinforcing their rhythmic coding (Frazee \& Kreuter, 1987), prudent within the fundamental implementation of the Orff-Schulwerk approach. Unfortunately, it was most likely lost in translation when having to formally notate the pitches for their bassline or sight-read the rhythmic notation of their melody's third measure. Moreover, the creative processes of Jane's group had interacted between and among themselves, as described in Wiggins (2003) model called the frame. The social context within these interactions contributed to a higher process of collaboration, which positively influenced their collective creative processes resulting in their collective creative product. 
Color Part. With regard to the color part compositional activity, three of the four stages relative to the creative processes emerged: preparation, illumination, and verification, with speculation that the incubation stage had emerged in relation to musical imagery. These creative compositional processes progressed in an interchangeably cyclical manner rather than a linear fashion, specifically between the stages of preparation, illumination, and verification. This was most likely due to the nature of the color part compositional activity having been formally introduced about 15-20 minutes within a 50-minute blocked period before having to perform all three Orff-based elements of their compositional project simultaneously for Ms. Smith. However, the creative processes of both Annie's and Jane's groups progressed in this interchangeably cyclical manner throughout each of the compositional activities as highlighted within Wiggins (2003) model called the frame, which states that various characteristics of composing such as organizing, evaluating, revising, refining, and rehearsing musical ideas interact between and among themselves. This interaction aligned with my findings that the creative processes of Annie's and Jane's groups, specifically their stages of preparation, illumination, and verification, overlapped with each other during this compositional activity. Strictly pertaining to Annie's group, enabling skills such as conceptual understanding with regards to rhythmic and formal notation were inherent within the context of creating their color part. Despite Nicole having written down the color part to play during the final performance, she displayed a lack of reading ability towards her formal notation of the color part by playing on every rest in their bassline ostinato. This could be a result of imposed time constraints to successfully progress through each of the creative process stages essential to Webster's (1990/2002) model of creative thinking in 
music. As for Jane's group, given the limited number of unpitched percussion instrumentation that Ms. Smith had in her possession, they settled for the rhythm sticks as their color part instrument, which was not their first choice. Moreover, this did not only create an environmental limitation known as an enabling condition within Webster's (1990/2002) model of creative thinking in music, but it also tampered with their preparation stage as they had to shift their planning in another direction since their favored unpitched percussion instrument was not available. Their intrinsic motivation was also impacted due to the lack of unpitched percussion instrumentation available to the group, indicating another enabling conditions within their preparation stage that contributed to their creative process as outlined in Webster's (1990/2002) model of creative thinking. As described by Hickey (2012), providing sufficient opportunities for exploring and manipulating various sound elements is crucial to children's creative thinking and sound-shaping, which are contributing factors to composition and improvisation. Conjointly, Springer (2013) stresses the importance of experiential learning through interactive engagement that promotes creative thinking, which is a fundamental component within the Orff-Schulwerk approach towards elemental musicmaking. By virtue of the Orff-based instruments being prone to facilitating music-making activities, children naturally gravitate towards recognizing familiar sounds through experiential learning implemented within the approach while reinforcing the rhythmic notation of sounds explored on the instruments. Alongside the creative processes that were unraveling in an interchangeably cyclical manner, musical imagery within the context of the group's incubation stage made an impact on the creation of their color part to the rest of the compositional project. Metaphorical analogies, such as "the little bit of 
ranch to your steak," served as musical imagery within their incubation stage, which assisted the progression between and among the specific stages of preparation, illumination, and verification. These metaphorical analogies also led to the natural progression of the verification stage in relation to the other respective Orff-based compositional activities. In spite of the noticeable absence of the incubation stage, my findings determined that the musical imagery within the context of the incubation stage experienced from the metaphorical analogy given did in fact reinforce the aforementioned creative processes by complementing their development. Hargreaves, MacDonald, and Miell (2012) claimed that the concept of imagination focuses on the “internal mental processes" that facilitates one's mind to produce a creative outcome, classifying one's imagination as a more inclusive term (as cited in Wang and Springer, 2013).

Throughout each of the Orff-based compositional activities, evidence within the findings determined that three of the four stages relative to the creative processes emerged: preparation, illumination, and verification. In addition, the incubation stage was speculated to have emerged in relation to musical imagery and rhythmic coding. Furthermore, enabling skills and enabling conditions were inherent within each student group throughout all three of the Orff-based compositional activities, contributing to their creative processes both in a positive and negative light. The findings suggest that, although each student group's creative compositional process did progress in a linear fashion from the beginning to the end of the study, their creative compositional processes throughout each of the compositional activities did progress in an interchangeably cyclical manner right until their final performances. Moreover, each student group 
experienced several enabling skills and enabling conditions during the progression of their creative compositional processes, which either worked in favor or against their Orffbased compositional activities while contributing to their overall creative compositional process.

Research Question Two: What are the children's perceptions of their own creative processes employed during an Orff-based composition task? Enabling conditions were most prominent within the children's perceptions of their creativity throughout the Orff-based compositional tasks as per my conversations with the student participants during our focus group interview. Explicitly, enabling conditions such as personalities, motivation, and environmental influences were determined to be contributing factors to their overall creative processes, both in a positive and negative light.

Enabling Conditions. Enabling conditions were discussed explicitly throughout the focus group interview. Student participants identified both positive and negative enabling conditions they experienced when employing in their Orff-based compositional tasks: 1) personality, 2) motivation, and 3) environmental influences. For example, Annie experienced several conflicts with her group member, Nicole. The two girls had several disagreements towards the compositional tasks due to the fact that Nicole commandeered the task cohesion albeit her curious and inventive nature. While in the focus group interview, Annie expressed that "Nicole was taking over everything and when I played the song once, just to show Ms. Smith, she was giving me the hard guilt trip and I do not like getting a hard guilt trip.... She kind of acted like the boss and there's no boss because it's a group composition." In addition to this conflict, Annie's group had several 
other hostile exchanges that tampered with the overall progression of their creative processes throughout each of the compositional tasks. The most perceptible being the loss of their melody sheet due to Nicole's negligence to turn it into Ms. Smith at the end of class the week prior. This caused great tension between Nicole and her peers, since the group had to recreate a melody from scratch with Ms. Smith scaffolding their exploration and refining of their melody. Annie expressed again: "[Nicole] lost the music, and then she was blaming it on me because that's just her. So we weren't really thinking about anything we were just like, 'let's get this over with.'” Several other student participants expressed afflicting situations they endured within their student groups due to differences in personalities, motivations, and environmental influences among their group members, but it was not as severe as the hostility exchanged by the group members within Annie's group. For example, Erika mentioned that she "liked working together to try to make a wonderful song," but added that "it was hard to agree on stuff and then making it work out." This was noticeably a positive contributing factor towards their group's social and environmental interactions given that each group member with different personalities came together and motivated each other towards creating their Orff-based composition task. In other words, where enabling conditions hindered certain student groups' creative processes, they stimulated other student groups' creative processes towards accomplishing their Orff-based composition task.

Webster's (1990/2002) model of creative thinking in music identifies several enabling conditions that contribute to the creative process that moves from divergent-toconvergent thinking. Springer (2013) states that "motivation, particularly intrinsic motivation, is one enabling condition that positively influences individuals' creative 
thinking and encourages creative achievement," (p. 172). Wiggins (2003) also states that "the nature of verbal, musical, and physical interaction that takes place within small groups or between pairs who are working together to create original musical ideas impacts both student process and product," (p. 160). In addition, Miell and MacDonald state that, although groups of friends and non-friends could produce similar qualities of music, those who work with friends do "significantly more to build on each other's musical ideas" to contribute towards their composition's development, as suggested by Erika's comments about the Orff-based compositional tasks during the focus group interview. Furthermore, Springer (2013) also mentioned that "many Orff activities are collaborative and social in nature, involving students in small groups as they sing, move, and play instruments," which naturally gravitates towards promoting creative thinking. However, this was not the case for several student groups, including Annie's group, since these enabling conditions did in fact tamper with their creative processes throughout the Orff-based composition tasks.

During the focus group interview, several enabling conditions such as personalities, motivations, and environmental influences were discussed in regards to children's creative compositional processes. These certain enabling conditions were determined to be contributing factors towards the children's creative processes both in a positive and negative light. The experience of social and environmental interactions within groups were expressed to be somewhat challenging at times for several student participants, but nonetheless manageable to complete the given compositional tasks for their overall Orff-based composition projects. However, confrontational disagreements within certain student groups did hinder the progression of their creative compositional 
processes while working through the compositional tasks, which to a degree had a negative impact on their overall Orff-based composition project.

\section{Research Question Three: What are the teacher's perceptions of teaching} and learning music composition through the Orff-Schulwerk approach? Enabling skills and enabling conditions were most prominent within the teacher's perception of teaching and learning music composition through the Orff-Schulwerk approach as discussed in our interview. Explicitly, enabling conditions within personal and social contexts were determined to be contributing factors towards the students' creative thinking. In addition, enabling skills, such as musical aptitude and conceptual understanding, were also determined to contribute to the students' creative thinking.

Teaching and Learning. Enabling skills and enabling conditions were discussed explicitly through the interview that I had with the teacher. The teacher identified that enabling skills and enabling conditions within the music-making process of the OrffSchulwerk approach had influenced her students' creative thinking and learning, both in a positive and negative way. During our interview, Ms. Smith elaborated on certain aspects of her warm-ups that are introduced as "little building blocks." These elemental building blocks are keen to her students' creative thinking and learning since they can be used to expand its simplicity on top of the other to reach a higher order of thinking, which is an important element in the music-making process within the approach's implementation. In addition, Ms. Smith elaborated that:

The idea of teaching rote first before putting visual notation to music... [that] is very Orff-y.... kids learn through play.... [and] the way that the instruments sound, using a bordun, all of those things are instant success for the kids, but then you can also elevate it by adding the melody and making it more complex... 
[these] basics that Orff gives you are instant [which] kids respond to it very quickly.

The elemental characteristics of rote teaching and learning prior to any visual/formal notation to music is a fundamental aspect of the Orff-Schulwerk approach, which is necessary towards expanding on larger-scaled tasks of music-making, namely composition and improvisation. Shamrock (1986) states that the Orff-Schulwerk approach is a pedagogical design for teachers to guide children through their musicmaking process including exploration, imitation, improvisation, and the creation of music, movement, speech, and drama, beginning with the most fundamental element and progressing to more complex elements. In relation, Landis and Carder (1972) states that children's creativity are expanded through their improvisations as they are encouraged to use compositional devices such as borduns, ostinatos, and rondos on Orff instrumentation that "offer[s] an interesting range of possibilities for playing [and improvising] the types of musical patterns" (p.87). These compositional devices are a necessary asset to the approach's music-making process since the essence of using repetitive rhythmic patterns on borduns, ostinatos, and rondos are consequently elemental, which also contributes towards the development of children's creative thinking and learning in music.

Ms. Smith also further elaborated that the exploration phase of the project was the shortest phase that the students experienced because they have had several opportunities to explore while working on other projects during the school year. However, she stated that the writing and revising stages "ended up being the longest portion of their project." Ms. Smith also mentioned that some of her students struggled with rehearsing through their creative products because "notation might have been an issue for some of them." In 
terms of the approach's implementation, this is counterintuitive to the study conducted by Frazee and Kreuter (1987) who states that Orff-based instruments could facilitate children's melodic composition through sound recognition while reinforcing rhythmic notation. Springer (2013) also elaborates that children's divergent and convergent thinking are enhanced through the deliberate and sufficient exploration of Orff instrumentation, aiding their creative thinking development. Springer added that several activities through the approach's implementation are “...collaborative and social in nature, involving students in small groups as they sing, move, and play instruments," which naturally gravitates towards promoting creative thinking among children. Additionally, Webster (1990/2002) states that several enabling skills and enabling conditions could influence the creative processes that move from divergent-to-convergent thinking. Annie's group was one of the few student groups that I speculated had a negative effect on the outcome of their creative product due to their environmental influences consisting of hostile exchanges and afflicting situations among the girls, thus resulting in a potential impact on their creative novelty, craftsmanship, and aesthetic sensitivity. This did not seem to be the case for other students groups and some of them even enjoyed the collaboration/social context of participating in a group-based project, which was mentioned several times throughout the focus group interview. Ms. Smith elaborated that:

... Working in groups is a big deal and I think that's hard for a lot of them... Orff is very group-based... I think the social interactions, that was probably a challenge for some of them, whether it was because they are used to taking control and they want to do everything or the opposite; they're shy and they don't know how to speak up and contribute to the process. 
During my interview with the teacher, enabling skills, such as musical aptitude and conceptual understanding, and enabling conditions within personal/social contexts were discussed to be contributing factors towards children's creative thinking and learning. It was evident that despite the students having the elemental building blocks implemented by the teacher in her daily musical instruction and the several experiential opportunities to explore on the Orff-based instruments during their music class, the progression of their creative thinking and learning were stagnant throughout various occasions when working on the compositional tasks, specifically during the stages of illumination and verification. In addition, several groups did struggle with sight-reading the formal rhythmic and melodic notation of their compositional tasks, which displayed a lack of musical aptitude and conceptual learning they possessed in spite of the teacher expressing their good rhythmic sight-reading skills. Moreover, several student groups also had strenuous experiences when having to collaborate and socialize with their group members due to the differences in personalities, motivations, and environmental influences, which resulted in arising conflicts among the group members. However, several other groups also experienced similar challenges when working through these compositional tasks yet embraced those differences to come together for the sake of their Orff-based composition projects.

Conclusion of Discussion. Throughout each of the Orff-based compositional tasks, evidence within the findings determined that three of the four stages in relation to the creative processes emerged, which were the preparation, illumination, and verification stages. In addition, the incubation stage was speculated to have emerged in 
relation to musical imagery and rhythmic coding, specifically during the melody and color part compositional tasks. Enabling skills and enabling conditions were also determined to be contributing factors towards the progression of the groups' creative compositional processes within the Orff-based compositional tasks, both in a positive and negative light. Pertaining to enabling skills, despite the children possessing the fundamental knowledge of the music-making process within the Orff-Schulwerk approach and having had an abundance of opportunities exploring on the Orff instrumentations, the progression of their creative processes were periodically stagnant during the course of all three of the Orff-based compositional tasks. Several groups also displayed a lack of musical aptitude and conceptual understanding due to their struggle with sight-reading the formal rhythmic and melodic notation of their compositional tasks, contrary to the teacher's expression of their good rhythmic sight-reading skills. In regards to enabling conditions, social and environmental interactions within the social context of the groups were determined to be both a positive and negative factor towards the children's creative processes. Children's differences in their personalities, motivations, and environmental influences resulted in several arising conflicts and disagreements among certain student groups, which hindered the progression of their creative compositional processes during the study. Nevertheless, the other student groups embraced these differences among themselves to come together to complete their Orffbased compositional projects. These findings suggest that, although each of the group's creative compositional processes did progress in a linear fashion from the beginning to the end of the study, their creative compositional processes did progress in an 
interchangeably cyclical manner throughout the course of the study until the final performances of their Orff-based compositional projects.

\section{Suggestions for Practitioners}

This descriptive qualitative study was determined to be subjectively singular with regards to the creative processes of elementary school students in music composition through the Orff-Schulwerk approach. The Orff-based compositional tasks given to the students allowed for their creative processes to progress in an interchangeably cyclical manner rather than a linear fashion, which most likely resulted from time constraints that the groups had to compose each of the Orff-based compositional elements (namely the bassline and color part) before combining these elements together for the final performances of their collective composition projects. In addition, these time constraints led the teacher to modify certain areas of her unit plan to accommodate those student groups who had not finished composing each of the compositional tasks as anticipated by the teacher when planning for this Orff-based composition project. When engaging children in any music-making activity, music educators must consider providing their students with sufficient musical experiences and boundless hands-on musical exploration that is both beneficial and enjoyable to children, especially when implementing the music-making process of the Orff-Schulwerk approach into their musical instruction. Furthermore, the practicality of removable key bars on pitched Orff instrumentations provides carefree engagement towards tonal exploration that facilitate activities such as song-writing and/or ostinato accompaniments to melodic compositions. However, music educators should consider each of their students' musical abilities and understanding 
prior to engaging them in a higher order thinking task such as formal notation of their musical exploration or combining several music-making elements to be played simultaneously. Although the approach's music-making process is naturally rudimental by including elemental building blocks through its pedagogical implementation, the role of the teacher within an Orff-based music classroom setting is imperative towards designing and facilitating these music-making activities to provide children with a sense of satisfaction, success, and enjoyment towards the experience they are actively engaging in. Through facilitating and providing music-making activities that are enjoyable and beneficial to children, music educators inspire children's creative authenticity and musical creativity as children gain confidence in their musical abilities while also enhancing their teaching approaches towards children's creativity in music composition, especially if implementing the pedagogical model of the Orff-Schulwerk approach into their musical instruction. Moreover, Orff activities are highly social and group-based in nature, therefore another factor that music educators should consider would be the differentiation of personalities and environmental influences among their students prior to assigning children into groups. Social conflicts and tension, aforementioned within Annie's group, could potentially tamper with children's ability to creatively think and/or learn due to enabling conditions that contribute towards their creativity. It is imperative that music educators take into consideration the social and environmental interactions of children within groups to facilitate the overall creative potential of their students when children engage in any music-making activity, explicitly when engaged in the musicmaking process within the Orff-Schulwerk approach. 


\section{Suggestions for Future Research}

The current descriptive qualitative study has determined that enabling skills and enabling conditions influenced the creative processes of children's music-making within the Orff-Schulwerk. Specifically, enabling skills and enabling conditions such as musical aptitude, conceptual understanding, motivation, personality, and environmental influences were contributing factors that impacted the children's creative thinking and learning during the study, both in a positive and negative manner. Further research on the process of music-making within the Orff-Schulwerk approach could be beneficial towards contributing to the development of children's musical experiences, musical abilities, and fundamental music learning, which could lead to children's musical and creative growth. In addition, by understanding the approach's music-making process, music educators can evolve their teaching strategies that could promote children's creative abilities and potential by enlightening and stimulating them as they continue to grow music-wise. However, additional research on the enabling skills and enabling conditions within the creative processes of children through the approach's implementation should also be thoroughly investigated. My reason in suggesting further investigation on these enabling skills and enabling conditions is highly predicated on my belief that this body of research could provide music educators with resourceful knowledge/skills that can be implemented into their musical instruction to facilitate their students' music-making process within the Orff-Schulwerk approach. Furthermore, this body of research could also provide music educators with efficient ways to mitigate negative social/environmental influences that can occur within groups during collaborative projects, which could impact children's overall musical and creative 
thinking/learning. Moreover, researching the music-making process within the OrffSchulwerk approach in tandem to the creative processes through a qualitative perspective could lead to an open-mindedness towards proceeding with the evaluation of children's creativity in a subjective-manner rather than an objective-manner. This qualitative perspective of children's music creativity through the approach's implementation can also provide music educators with insight towards evaluating children's creative processes versus their creative products since evaluating the creative outcome does not necessarily perceive the overall creative processes that led to the final creative product. Subsequently, by further examining the creative processes instead of the creative products through a qualitative lens within the Orff-Schulwerk could provide further qualitative insight to build on the body of qualitative research of children's musical creativity through the music-making process of the approach's implementation. 


\section{References}

American Orff-Schulwerk Association. (n.d.) AOSA Teacher Education Courses. Retrieved from https://aosa.org/professional-development/teacher-education-coursessummary/

Arieti, S. (1976). Creativity: A magic synthesis. New York, New York: Basic Books, Inc., Publishers.

Beegle, A., \& Bond, J. (2016). Orff Schulwerk: Releasing and Developing the Musical Imagination. In C. R. Abril, \& B. M. Gault (Eds.), Teaching general music: Approaches, issues, and viewpoints (pp. 25-48). New York, NY: Oxford University Press.

Choksy, L., Abramson, R. M., Gillespie, A. E., Woods, D., \& York, F. (2001). Teaching Music in the Twenty-First Century. Upper Saddle River, New Jersey: Prentice-Hall, Inc.

Churchley, F. (1980). Contemporary approaches in music education. McGill Journal of Education, 15(3), 45-48.

Creswell, J. W. (2014). Research design: Qualitative, quantitative, and mixed methods approaches. Thousand Oaks, California: SAGE Publications, Inc.

Davenport, C. (2016). A model of classroom environment and social-psychological factors influencing computer-based compositional creativity (Doctoral dissertation, University of Miami). Open Access Dissertations. Retrieved from https://scholarship.miami.edu/discovery/fulldisplay/alma991031447282902976/01UOML _INST:ResearchRepository

Fraenkel, J. R., Wallen, N. E., \& Hyun, H. H. (2012). How to design and evaluate research in education. New York, New York: McGraw-Hill Companies, Inc.

Frazee, J., \& Kreuter, K. (1987). Discovering Orff: A curriculum for music teachers. New York, New York: Schott Music Corporation.

Hickey, M. (Ed.). (2003). Why and how to teach music composition: A new horizon for music education. Reston, Virginia: MENC: The National Association for Music Education.

Hickey, M. (2012). Music outside the lines: Ideas for composing in K-12 music classrooms. Oxford, New York: Oxford University Press.

Kratus, J. (1989). A time analysis of the compositional processes used by children ages 7 to 11. Journal of Research in Music Education, 7(1), 5-20.

Landis, B., \& Carder, P. (1972). The eclectic curriculum in American music education: Contributions of Dalcroze, Kodaly, and Orff. Washington, District of Columbia: Music Educators National Conference. 
Long, A. (2013). Involve me: Using the Orff approach within the elementary classroom. 68 Retrieved from

http://thekeep.eiu.edu/cgi/viewcontent.cgi?article=1003\&context=lib_awards_201 3_docs

Mark, M. L., \& Gary, C. L. (2007). A history of American music education. Blue Ridge Summit, PA: Rowman \& Littlefield Education.

Miner, B. (2007). Fostering musical creativity in the elementary classroom. University of New Hampshire Scholars' Repository, 26(1), 1-8.

Orff, C. (1963). The Schulwerk: Its origin and aims. Music Educators Journal, 49(5), 6974.

Shehan, P. K. (1986). Major approaches to music education: An account of method. Music Educators Journal, 72(6), 26-31.

Springer, D. G. (2013). Creative thinking in Orff Schulwerk. In C. C. Wang, \& D. G. Springer (Eds.), Orff Schulwerk: Reflections and directions. Chicago, Illinois: GIA Publications, Inc.

Thresher, J. M. (1964). The contributions of Carl Orff to elementary music education. Music Educators Journal, 50(3), 43-48.

Wang, C. C., \& Springer, D. G. (2013). Orff Schulwerk: Reflections and directions. Chicago, Illinois: GIA Publications, Inc.

Webster, P. R. (1990). Creativity as creative thinking. Music Educators Journal, 76(9), 22-28.

Wiggins, J. (2003). A frame for understanding children's compositional processes. In Hickey, M. (Ed.), Why and how to teach music composition: A new horizon for music. Reston, Virginia: MENC: The National Association for Music Education.

Womack, S. C. T. (2008). A comparison of the effects of Orff Schulwerk and traditional music instruction on selected elements of music achievement in third, fourth, and fifth grade students (Doctoral dissertation, University of Georgia). Retrieved from https://getd.libs.uga.edu/pdfs/womack_sara_c_200808_edd.pdf 
APPENDIX A

\section{FOCUS GROUP INTERVIEW QUESTIONS}




\section{FOCUS GROUP INTERVIEW QUESTIONS}

Personal enjoyment

1. What did you enjoy most/least about the composition group project that you guys worked on these last few weeks?

Creative processes

2. What were some of the thoughts/ideas that you and your group wanted to explore while working on the bassline / melody / color part? What thoughts/ideas did you go with for the bassline / melody / color part and why?

3. From among the bassline, melody, and color part activities, which one would you consider to be the easiest/hardest to work through and why?

\section{Orff-based}

4. What were some of the easy/hard characteristics of working through the project using the Orff instruments? Why were those characteristics easy/hard? 
APPENDIX B

\section{TEACHER INTERVIEW QUESTIONS}




\section{TEACHER INTERVIEW QUESTIONS}

\section{Orff-based}

1. What are your thoughts and experiences on teaching music through the OrffSchulwerk approach?

2. What would you say you like most/least about teaching music through the OrffSchulwerk approach?

3. What would you say is the most/least enjoyable part of learning for your students through the approach's implementation and why?

\section{Composition}

4. How do you typically approach teaching music composition using the approach's implementation?

5. Do you find it easier or harder to teach music composition using the approach's implementation and why?

6. In your opinion, which of the compositional tasks/elements of the project conveyed the most creativity/creative processes in your students and why?

7. In your opinion, which of the compositional tasks/elements did your students most/least enjoy experiencing while working on this project and why?

\section{Innovating Music Education}

8. What are your beliefs on music education having to transition towards innovative technological advances present nowadays?

9. Do you think teaching approaches, such as the Orff-Schulwerk and many others, used by music educators will suffer to maintain its relevance in music education as a result of innovative technological advances in schools and why?

10. Where would you incorporate technology best into your established Orff-based teaching style? Why would you incorporate technology in that/those specific $\operatorname{area}(\mathrm{s})$ ? 
APPENDIX C

\section{RECRUITMENT INFORMATION LETTER}




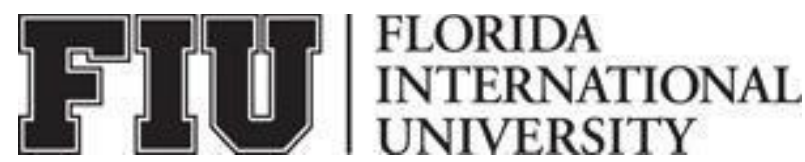

INFORMATIONAL LETTER

Children's Creative Processes in Music Composition Through the Orff-Schulwerk Approach

Hello, my name is Ashley Viera. You have been chosen to evaluate if one or more of your music classes can participate in a research study about children's creativity in music composition. The purpose of this study is to explore children's creativity in music composition through the Orff-Schulwerk approach. If you decide that your music classes qualify to be in this study, you and your music classes will be the only participants in this research study. Participation in this study will take approximately six weeks of your time. If you agree that your music classes can participate in the study, I will ask you to do the following things:

1. Have your students [the student participants] partake in a music composition activity on Orff-based instruments while I observe their processes of executing the activity in the background. This initial part of the study will last five weeks.

2. The last week will involve interviews with you and focus groups from your classes. During the interview process, you will be encouraged to share your teaching experience and thoughts about the compositional activity. For the focus group student participants, they will be encouraged to share their thoughts and experiences of the compositional activity, as well.

There are no foreseeable risks or benefits to your participating classes in this study. However, minimal risks to both you and your student participants may occur, but no more than those ordinarily encountered in daily school life routines and procedures. It is expected that this study will benefit the lack of research regarding children's creativity in music composition through the Orff-Schulwerk approach. In addition, this study can benefit researchers and practitioners, like yourself, by contributing to a better understanding of children's creative processes and enhance teaching approaches in regards to musical creativity. Note: I have attached the study's research overview for your reference.

There is no cost or payment to you and your participating classes. If you have questions while taking part, please stop me and ask.

You and your participating classes will remain anonymous. To secure you and your participating classes' privacies, I will adhere to the policies abided by Florida International University Institutional Review Board (IRB) and ensure meeting the outlined issues of consent. 
If you have questions or concerns, you may contact me at (786) 380-8452 or avier026@fiu.edu.

If you would like to talk with someone about you or your classes' rights of being a participant in this research study or about ethical issues with this research study, you may contact the FIU Office of Research Integrity by phone at 305-348-2494 or by email at ori@fiu.edu.

You and your classes' participation in this research is voluntary, and you will not be penalized or lose benefits if you refuse to participate or decide to stop participating in the study. You may keep a copy of this form for your records.

\section{Research Overview}

The Orff-Schulwerk approach has gained much global recognition since it arrived in the United States. Its fundamental approach has demonstrated to be a valuable contribution to developing children's learning in music education. Explicitly, the process of music-making within the Orff-Schulwerk approach has proven to be useful to children's musical experiences and fundamental understanding while stimulating their creative ability and potential (Choksy et al., 2001; Churchley, 1980; Hickey, 2003, 2012; Orff \& Walter, 1963; Shehan, 1986; Thresher, 1964; Wang \& Springer, 2013).

Throughout the Orff-Schulwerk approach, children are continuously encouraged to immerse themselves in "playful musical exploration" and "creative expression" (Beegle \& Bond, 2016) as they navigate through their creative thought processes. The depiction of musical ideas in composition allows children to freely express their musical thinking while demonstrating their conceptual music understanding (Wiggins, 2003). Through the subsequent act of composing and improvising, children may develop their musical identity as they immerse themselves within the compositional process, directly enhancing their musical experiences and musicality while inspiring their creative novelty (Hickey, 2012). Considering the divergent-to-convergent process through the stages of preparation, incubation, illumination, verification (Webster, 1990) allows children to display their musically and creatively inclined skills by achieving compositional outcomes. Children with continuous exposure to composition within specific parameters are more likely to develop satisfying musical compositions while inadvertently improving their creativity. Furthermore, children also demonstrate the collective work of their divergent and convergent thought processes when given substantial opportunities to compose musically (Wang \& Springer, 2013).

Despite enhancing children's musicality, musical experience, and creativity, there is an insufficient amount of data examining their creative processes in music composition through the Orff-Schulwerk approach (Hickey, 2003, 2012; Orff \& Walter, 1963; Thresher, 1964; Webster, 1990; Womack, 2008). The implementation of composition inspires children's creativity and improvisation, as discussed in multiple sources examined in this study, with a growing area of research on children's creativity using 
Orff instrumentation during compositional activities (Choksy et al., 2001; Wang \& Springer, 2013; Womack, 2008). However, further knowledge on musical creativity through the compositional process could contribute to a better understanding of children's creative thought and enhance teaching strategies. Its implementation on children's musical creativity can provide music educators with the opportunity to strengthen their educational approach to composition, further facilitating and nurturing children's independent thinking while encouraging their creative authenticity through music-making (Hickey, 2003, 2012). 
APPENDIX D

\section{PARENTAL CONSENT FORM}




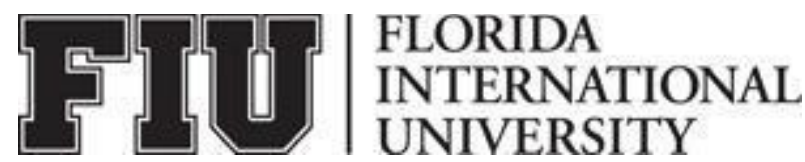

PARENTAL CONSENT TO PARTICIPATE IN A RESEARCH STUDY

Children's Creative Processes in Music Composition Through the Orff-Schulwerk

Approach

\section{SUMMARY INFORMATION}

Things you should know about this study:

- Purpose: The purpose of the study is to explore the children's creativity in music composition through the Orff-Schulwerk approach.

- Procedures: If you choose to allow your child to participate, your child will be asked to create their individual composition using respective Orff instrumentations during their composition project.

- Duration: This will take about six weeks.

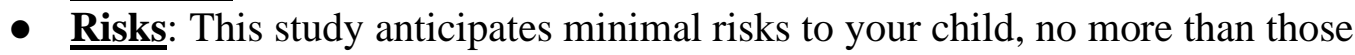
ordinarily encountered in daily school life routines and procedures.

- Benefits: There are no benefits to your child from this research, except for your child to enjoy their composition project that they will work on during the study.

- Alternatives: There are no known alternatives available to your child other than not taking part in this study.

- Participation: Taking part in this research project is voluntary.

Please carefully read the entire document before agreeing to participate.

\section{PURPOSE OF THE STUDY}

The purpose of the study is to explore the children's creativity in music composition through the Orff-Schulwerk approach.

\section{NUMBER OF STUDY PARTICIPANTS}

If you agree to allow your child to participate in this study, he/she will be one of the students to participate in this research study.

\section{DURATION OF THE STUDY}

Your child's participation will involve six weeks of their time during school hours.

\section{PROCEDURES}

If your child participates in this study, we will ask your child to do the following things:

1. Complete a composition project using specific Orff instrumentations. 
a. Your child will be audio taped so that we may write out their responses and comments during the project at a later time and used to analyze the data recorded by the researcher.

2. If your child is selected to be part of an interview group, they will be encouraged to talk openly about their thoughts and experiences that they had while working on their composition project.

\section{RISKS AND/OR DISCOMFORTS}

We anticipate minimal risks that your child may encounter during the study, but no more than those ordinarily encountered in daily school life routines and procedures.

\section{BENEFITS}

The study has no possible benefits to your child except for your child to enjoy the composition project that they will work on during the study.

\section{ALTERNATIVES}

There are no known alternatives available to your child other than not taking part in this study.

\section{CONFIDENTIALITY}

The records of this study will be kept private and will be protected to the fullest extent provided by law. In any sort of report we might publish, we will not include any information that will make it possible to identify your child. Research records will be stored securely and only the researcher team will have access to the records. However, your child's records may be inspected by authorized University or other agents who will also keep the information confidential.

In addition, we will adhere to the policies abided by Florida International University Institutional Review Board (IRB) to ensure meeting the outlined issues of consent.

If we learn about serious harm to you or someone else, we will take steps to protect the person endangered even if it requires telling the authorities without your permission. If we have reason to believe that your child is being abused, we will report this to the Florida Abuse hotline. In these instances, we would only disclose information to the extent necessary to prevent harm.

\section{USE OF YOUR CHILD'S INFORMATION}

Your child's information collected as part of the research will not be used or distributed for future research studies even if identifiers are removed. 


\section{COMPENSATION \& COSTS}

Your child will not receive a payment for their participation. In addition, there are no costs to your child for participating in this study.

\section{RIGHT TO DECLINE OR WITHDRAW}

Your child's participation in this study is voluntary. Your child is free to participate in the study or withdraw his/her consent at any time during the study. The investigator reserves the right to remove your child from the study without your consent at such time that he/she feels it is in the best interest.

\section{RESEARCHER CONTACT INFORMATION}

If you have any questions about the purpose, procedures, or any other issues relating to this research study you may contact Ashley Viera at (786) 380-8452 or avier026@fiu.edu.

\section{IRB CONTACT INFORMATION}

If you would like to talk with someone about your child's rights of being a subject in this research study or about ethical issues with this research study, you may contact the FIU Office of Research Integrity by phone at 305-348-2494 or by email at ori@fiu.edu.

\section{PARTICIPANT AGREEMENT}

I have read the information in this consent form and agree to allow my child to participate in this study. I have had a chance to ask any questions I have about this study, and they have been answered for me. I understand that I will be given a copy of this form for my records.

Signature of Parent/Guardian

Printed Name of Parent/ Guardian

Printed Name of Child Participant

Signature of Person Obtaining Consent
Date

Date 
APPENDIX E

ADULT TEACHER CONSENT FORM 


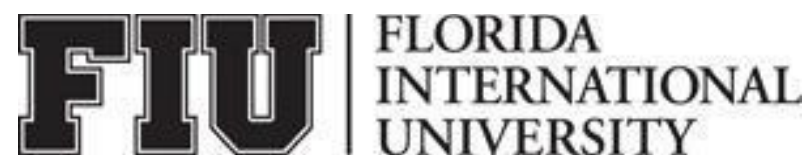

ADULT CONSENT TO PARTICIPATE IN A RESEARCH STUDY

Children's Creative Processes in Music Composition Through the Orff-Schulwerk

Approach

\section{SUMMARY INFORMATION}

Things you should know about this study:

- Purpose: The purpose of the study is to explore the children's creativity in music composition through the Orff-Schulwerk approach.

- Procedures: If you choose to participate, you will be asked to give your students a music composition activity on Orff-based instruments.

- Duration: This will take about six weeks.

- $\underline{\text { Risks: }}$ The study is anticipated to have minimal risks to the teacher and student participants, no more than those ordinarily encountered in daily school life routines and procedures.

- Benefits: The main benefit to you from this research is that it can contribute to a better understanding of children's creative processes which can enhance your teaching approach with regards to musical creativity.

- Alternatives: There are no known alternatives available to you other than not taking part in this study.

- Participation: Taking part in this research project is voluntary.

Please carefully read the entire document before agreeing to participate.

\section{PURPOSE OF THE STUDY}

The purpose of the study is to explore the children's creativity in music composition through the Orff-Schulwerk approach.

\section{NUMBER OF STUDY PARTICIPANTS}

If you decide to be in this study, you and your music classes will be the only participants in this research study.

\section{DURATION OF THE STUDY}

Your participation will involve six weeks of your time.

\section{PROCEDURES}

If you agree to be in the study, we will ask you to do the following things: 
1. Give your students a music composition activity to complete on Orff-based instruments while I observe their processes of executing the activity in the background. As I observe from the background, I will be taking notes and audio taping the students' comments and/or responses during the activity so I may transcribe it later for my use to analyze the data. This initial part of the study will last five weeks.

2. You and focus groups of your participating classes selected by you will be interviewed on the last week of the study.

a. As the teacher, you will be encouraged to share your thoughts and teaching experiences of the compositional activity in a way that is convenient and most comfortable to you.

$b$. Your students that will be in the focus groups will be encouraged to share their thoughts and experiences of the compositional activity in a way that is safe and comfortable to them.

\section{RISKS AND/OR DISCOMFORTS}

We anticipate risks to both you, the teacher, and student participants to be minimal, or no more than those ordinarily encountered in daily school life routines and procedures.

\section{BENEFITS}

The study has the following possible benefits to you: as a teacher, understanding children's creative compositional processes through the Orff-Schulwerk approach can enhance your own teaching approach with regards to musical creativity. In addition, it may benefit the lack of research regarding children's creativity in music composition through the Orff-Schulwerk approach.

\section{ALTERNATIVES}

There are no known alternatives available to you other than not taking part in this study.

\section{CONFIDENTIALITY}

The records of this study will be kept private and will be protected to the fullest extent provided by law. In any sort of report we might publish, we will not include any information that will make it possible to identify you or the identity of your participating classes. Research records will be stored securely, and only the researcher team will have access to the records. However, your records may be inspected by authorized University or other agents who will also keep the information confidential.

In addition, we will adhere to the policies abided by Florida International University Institutional Review Board (IRB) and ensure to meet the outlined issues of consent. 


\section{USE OF YOUR INFORMATION}

You and your participating classes' information collected as part of the research will not be used or distributed for future research studies even if identifiers are removed.

\section{COMPENSATION \& COSTS}

There are no costs or compensations associated with you and your classes' participation in this study.

\section{RIGHT TO DECLINE OR WITHDRAW}

Your participation in this study is voluntary. You are free to participate in the study or withdraw your consent at any time during the study. The investigator also reserves the right to remove you without your consent at such time that he/she feels it is in the best interest.

\section{RESEARCHER CONTACT INFORMATION}

If you have any questions about the purpose, procedures, or any other issues relating to this research study you may contact Ashley Viera at (786) 380-8452 or avier026@fiu.edu.

\section{IRB CONTACT INFORMATION}

If you would like to talk with someone about your rights of being a subject in this research study or about ethical issues with this research study, you may contact the FIU Office of Research Integrity by phone at 305-348-2494 or by email at ori@fiu.edu.

\section{PARTICIPANT AGREEMENT}

I have read the information in this consent form and agree to participate in this study. I have had a chance to ask any questions I have about this study, and they have been answered for me. I understand that I will be given a copy of this form for my records.

Signature of Participant

Date

Printed Name of Participant

Signature of Person Obtaining Consent

Date 
APPENDIX F

\section{MINOR ASSENT FORM}




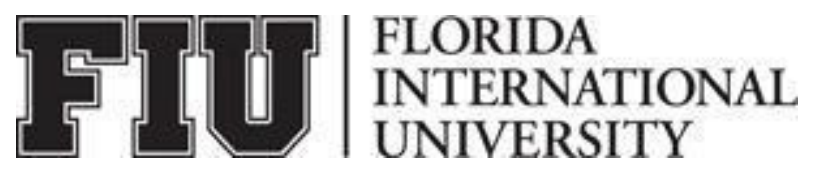

CHILD ASSENT TO PARTICIPATE IN A RESEARCH STUDY

Children's Creative Processes in Music Composition Through the Orff-Schulwerk

Approach

\section{WHY ARE YOU DOING THIS STUDY?}

We would like for you to be in a research study we are doing. A research study is a way to learn information about something. We would like to find out more about children's creativity in music composition through an approach called the Orff-Schulwerk.

\section{HOW MANY OTHERS WILL BE IN THIS STUDY?}

If you agree to participate in this study, you will be one of the students in your class to participate in this research study.

\section{WHAT WILL HAPPEN IN THIS STUDY?}

If you participate in this study, we will ask you to do the following things:

1. Complete a composition project on specific Orff instrumentations.

a. You will be audio taped so that we may write out your responses and comments during the project at a later time.

2. If you are selected to be part of an interview group, you will be encouraged to talk openly about your thoughts and experiences that you had while working on your project.

\section{HOW LONG WILL THE STUDY LAST?}

Your participation will require six weeks of your time during school hours.

\section{CAN ANYTHING BAD HAPPEN TO ME?}

You should not feel like you are uncomfortable at all while participating in this study, but if you do all you have to do is let us know and we can remove you to a place where you feel comfortable at.

\section{CAN ANYTHING GOOD HAPPEN TO ME?}

You may benefit from participating in this study by having a great time learning and exploring music on different Orff instruments while you compose your own musical composition! 


\section{DO I HAVE OTHER CHOICES?}

There are no known alternatives available to you other than not taking part in this study. If you decide you would rather not participate in the study, that is your right and decision and we will respect it.

\section{WILL ANYONE KNOW I AM IN THE STUDY?}

The records of this study will be kept private and will be protected by the researchers. We will not use your name or identity when we talk or write about your participation in this study.

\section{WILL I BE GIVEN ANYTHING FOR PARTICIPATING?}

You will not receive a payment for your participation. You will get a grade for your composition project by your teacher once you complete it. You will not need to pay for anything to participate in this study.

\section{WHAT IF I DO NOT WANT TO DO THIS?}

You do not have to be in this study if you do not want to and you can quit the study at any time. If you do not like a question, you do not have to answer it and, if you ask, your answers will not be used in the study. No one will get mad at you if you decide you do not want to participate.

\section{WHO CAN I TALK TO ABOUT THE STUDY?}

If you have any questions about the research study, you may contact Ashley Viera at (786) 380-8452 or avier026@ fiu.edu. If you would like to talk with someone about your rights of being a participant in this research study, you may contact the FIU Office of Research Integrity by phone at 305-348-2494 or by email at ori@ fiu.edu.

\section{PARTICIPANT AGREEMENT}

This research study has been explained to me and I agree to be in this study.

Signature of Child Participant

Date

Printed Name of Child Participant

Signature of Person Obtaining Consent

Date 
APPENDIX G

AUTHORIZATION FOR RECORDING WITH INFORMATION LETTER 


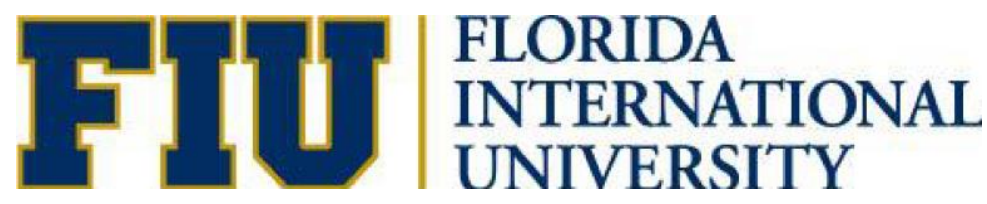

\section{Photographic/Film Consent \& Release Form}

I hereby give my consent to Ashley Viera

and The Florida International University Board of Trustees ("FIU"):

(a) to record my child's likeness and voice on a video, audio, photographic, digital, electronic or any other medium and to use my child's name in connection with these recordings; and

(b) to use, reproduce, exhibit or distribute these recordings in whole or in part in perpetuity in any and all media throughout the universe (including but not limited to print publications, video tapes, non-theatrical, home video, CD-ROM, internet and any other electronic or other medium presently in existence or invented in the future) for any purpose that FIU, and those acting pursuant to its authority, deem appropriate, including promotional, recruiting, advertising and any commercial or non-commercial use.

I hereby release Ashley Viera and FIU from and against any and all claims, demands, actions, causes of actions, suits, costs, expenses, liabilities, and damages whatsoever that I may hereafter have from liability for any violation of any personal or proprietary right I may have in connection with such use of my child's likeness, voice, or name in any medium, and expressly waive any rights to privacy I may have under the Family Educational Rights and Privacy Act ("FERPA") and/or \$1002.22, Fla. Stat. I understand and agree that all such recordings, in whatever medium, shall remain the property of FIU.

I have read and fully understand the terms of this release.

Sign: Date:

Name:

Address:

City/State/Zip:

Phone:

Email:

Youth under 18 must have a parental signature. 


\section{Children's Creative Processes in Music Composition Through the Orff-Schulwerk Approach}

\section{Consent to Audio Record Conversations and/or Interviews:}

Hello, my name is Ashley Viera and I am involved in a research study called Children's Creative Processes in Music Composition Through the Orff-Schulwerk Approach with my professor, Candice Davenport-Mattio, at Florida International University (FIU).

PURPOSE OF THE STUDY: The purpose of this study is to explore children's creativity in music composition through the Orff-Schulwerk approach. With the help of your child, I will learn more about their creative processes through the Orff-Schulwerk approach and I will be able to contribute to a growing body of research on children's creativity through the Orff-Schulwerk approach related to music composition.

With your permission, I will be recording the conversations between your child and myself in order to reproduce and write their answers later for research purposes. If your child is selected to be part of one of the few interview groups, I will also be recording the conversations during that interview. Please note that you have the right to review and retain these recordings or choose not to opt-out of your child being recorded at all. Either way, you can still let your child participate in this study.

Your child's participation involves minimal risks and benefits to you and your child, and is completely voluntary. You can deny that your child participates in this study or that your child stops participating in this study at any time if you wish to do so without any negative consequences to you or your child.

After reading this letter, if you grant me your consent to record the conversations between your child and myself for research purposes, please sign the form entitled "Photographic/ Film Consent and Release Form."

Thanks for your time and consideration,

Respectfully,

Ashley Viera

(786) 380-8452

Avier026@fiu.edu 